Illinois State University

ISU ReD: Research and eData

Theses and Dissertations

9-30-2014

\title{
Articulating the Experiences and Perceptions of Graduates From An Inner City Field-Based Teacher Preparation Program: A Sociocritical Perspective
}

Cynthia Lucretia Thompson

Illinois State University, clthomp@ilstu.edu

Follow this and additional works at: https://ir.library.illinoisstate.edu/etd

Part of the Curriculum and Instruction Commons, and the Teacher Education and Professional Development Commons

\section{Recommended Citation}

Thompson, Cynthia Lucretia, "Articulating the Experiences and Perceptions of Graduates From An Inner City Field-Based Teacher Preparation Program: A Sociocritical Perspective" (2014). Theses and Dissertations. 287.

https://ir.library.illinoisstate.edu/etd/287

This Dissertation is brought to you for free and open access by ISU ReD: Research and eData. It has been accepted for inclusion in Theses and Dissertations by an authorized administrator of ISU ReD: Research and eData. For more information, please contact ISUReD@ilstu.edu. 


\title{
ARTICULATING THE EXPERIENCES AND PERCEPTIONS OF GRADUATES FROM AN INNER CITY FIELD-BASED \\ TEACHER PREPARATION PROGRAM: \\ A SOCIOCRITICAL PERSPECTIVE
}

\author{
Cynthia L. Thompson
}

205 Pages

May 2015

Teacher preparation has been the center of educational reform in the United States for many years. A review of literature proposes that many universities are addressing the educational challenge of developing stronger teacher preparation programs by collaborating with neighborhood schools to implement field-based teacher preparation programs. To improve programs of this nature, the experiences and perceptions of graduates who participated in the program must be explored. A number of recommendations for educational reform exist on the topic of teacher preparation, but much-needed research must be done on the experiences and perceptions of participants in field-based teacher preparation programs. Current research on field-based teacher preparation pinpoints a critical need to identify whether these programs prepare teachers adequately for the teaching profession. Previous research reminds us of the importance of informing educational policymakers, state boards of education, schools of education and the wider educational community in the continued development of field-based teacher preparation programs nationwide. 


\section{ARTICULATING THE EXPERIENCES AND PERCEPTIONS OF GRADUATES FROM AN INNER CITY FIELD-BASED \\ TEACHER PREPARATION PROGRAM: \\ A SOCIOCRITICAL PERSPECTIVE}

CYNTHIA L. THOMPSON

A Dissertation Submitted in Partial

Fulfillment of the Requirements for the Degree of

DOCTOR OF EDUCATION

School of Teaching and Learning

ILLINOIS STATE UNIVERSITY 


\section{ARTICULATING THE EXPERIENCES AND PERCEPTIONS OF GRADUATES FROM AN INNER CITY FIELD-BASED TEACHER PREPARATION PROGRAM: A SOCIOCRITICAL PERSPECTIVE}

CYNTHIA L. THOMPSON

COMMITTEE MEMBERS:

Barbara Meyer, Chair

Sandra Westbrooks

Miranda Lin 
Copyright 2015 Cynthia L. Thompson 


\section{ACKNOWLEDGMENTS}

This dissertation is dedicated to my dad William Thompson Jr.; mom Baulkus Chula Heard; Aunt Lucretia Gale Heard; Uncle Antanasio Resendez; and Dr. Ward Weldon, my mentor and close friend, who encouraged me while in graduate school at the University of Illinois/Chicago and afforded me the opportunity to work as his assistant in the "Principal's Summer Institute" and on our research project in Africa. All encouraged me to pursue a doctorate degree and later passed during my journey.

Thank you to my committee members, Dr. Barbara Meyer, Dr. Sandra Westbrooks, and Dr. Miranda Lin. Thank-you Dr. Athanase Gahungu for the many conversations and guidance regarding my research. The daily phone calls from my close friends Rudy Sagers, Tonya Johnson, Yolanda Thompson, Levetrice Gant, and Sharon Rushing for keeping me encouraged, focused, and motivated. My Uncle Ahbran Resendez for your ongoing words of wisdom, encouragement, love, and support.

I would like to express my deepest gratitude to: my dissertation chair Dr. Barbara Meyer, and Dr. Amy Hurd, Graduate School Director, for their ongoing support and patience; Dr. Haling, for supporting me and affording me the opportunity to serve as the student representative on the Graduate Council for the College of Education; and Dr.

Lydia Kyei-Blankson for assisting in my development and understanding as a qualitative researcher in analyzing and reporting qualitative data. Thank you all for your ongoing support and positive words of encouragement over the past year. 
Dr. Westbrooks, I want to take this opportunity to let you know how much I respect and admire you as a friend, mentor, and professional. The knowledge and wisdom you have imparted to me has played a major role through my career. I believe my success is due, at least in part, to your ongoing encouraging words, sincere support, and telling me: "Do not stop at a Bachelor's Degree. Go all the way and earn a Doctorate Degree." Even though you were incredibly busy, you have always been there to assist me. You have taught me how to break through many barriers to accomplish my goals and how to confront difficult challenges with intelligence, patience, and perseverance. I truly appreciate and value everything I have acquired from you.

Thank you for supporting my interest in international education and allowing me to travel to Nakajo, Japan, to conduct research entitled "A Comparative Analysis of the Educational System in Japan to Chicago, Illinois." Most importantly, thank you for being a great mentor and always keeping the door open to engage in dialogue with me. You have been a great role model and an inspiration in my development as a professional and researcher.

C. L. T. 


\section{CONTENTS}

Page

ACKNOWLEDGMENTS

CONTENTS

iii

TABLES viii

FIGURES

CHAPTER

I. THE PROBLEM AND ITS BACKGROUND

Background of the Study $\quad 2$

$\begin{array}{ll}\text { Statement of the Problem } & 6\end{array}$

Nature and Purpose of the Study 9

Research Question $\quad 11$

\begin{tabular}{lr} 
Significance of the Study & 12 \\
\hline
\end{tabular}

$\begin{array}{ll}\text { Theoretical Foundations } & 14\end{array}$

$\begin{array}{ll}\text { Definition of Terms } & 18\end{array}$

$\begin{array}{ll}\text { Conclusion } & 20\end{array}$

II. LITERATURE REVIEW 22

Field-Based Defined 23

Prior Experience $\quad 24$

Field-Experience $\quad 25$

Historical Perspective $\quad 26$

Need for Study $\quad 41$

Interpretivism Literature Review $\quad 42$

Interpretivism: A Working Definition 42

Interpretivism as Related to Education $\quad 45$

Interpretivism as Related to This Research 46

Social Interpretivism 49

Critical Interpretivism $\quad 51$ 
Social Critical Interpretivism $\quad 54$

Sociocritical Interpretivism $\quad 55$

Language, Culture, and Experience $\quad 58$

Language, Culture, and Experience (LCE) in the Community 61

$\begin{array}{ll}\text { Conclusion } & 62\end{array}$

$\begin{array}{ll}\text { III. METHODOLOGY } & 64\end{array}$

Research Design $\quad 65$

Tenets of Phenomenology 66

Listening $\quad 66$

Conversation $\quad 66$

Validating the Experience $\quad 67$

Describing the Experience $\quad 67$

Interpreting and Analyzing the Experience $\quad 68$

Disclosure of Data $\quad 68$

Researcher Bias $\quad 68$

Field-Based Participants $\quad 69$

University Background $\quad 71$

Participants' Background 71

Data Collection Procedures 73

$\begin{array}{ll}\text { Interviewing } & 75\end{array}$

Kvale's Seven Stages of Interviewing $\quad 75$

Seidman's Three-Stage Interview Process 77

Data Collection $\quad 80$

Data Analysis $\quad 82$

$\begin{array}{lr}\text { Saturation } & 83\end{array}$

Juxtaposition $\quad 83$

Reliability and Validity $\quad 84$

$\begin{array}{ll}\text { Reliability } & 84\end{array}$

Transcriptions $\quad 85$

Coding $\quad 85$

Crosschecking $\quad 86$

$\begin{array}{ll}\text { Validity } & 86\end{array}$ 
$\begin{array}{ll}\text { Transcription } & 87\end{array}$

Member Checking $\quad 88$

$\begin{array}{ll}\text { Description } & 88\end{array}$

Clarifying Bias $\quad 88$

Adverse Data $\quad 89$

Time $\quad 89$

Internal Validity $\quad 89$

Ethical Considerations $\quad 90$

Limitations of the Methodology 91

Conclusion 93

IV. ANALYSIS OF RESEARCH 95

Developed Themes $\quad 98$

$\begin{array}{ll}\text { Perceptions } & 100\end{array}$

$\begin{array}{ll}\text { Daily Challenges } & 101\end{array}$

$\begin{array}{ll}\text { Experience } & 102\end{array}$

Protective Behavior 106

Positive or Negative Experience 107

$\begin{array}{ll}\text { Volunteerism } & 109\end{array}$

$\begin{array}{ll}\text { Life Experiences } & 110\end{array}$

$\begin{array}{ll}\text { Preparation } & 112\end{array}$

$\begin{array}{ll}\text { One-on-One Mentoring } & 117\end{array}$

As a First Year Teacher $\quad 117$

Understanding their Role as an Educator 119

$\begin{array}{ll}\text { Teacher Efficacy } & 121\end{array}$

$\begin{array}{ll}\text { Collaboration } & 122\end{array}$

$\begin{array}{ll}\text { Disposition } & 124\end{array}$

$\begin{array}{ll}\text { Knowledge and Skills } & 124\end{array}$

Collaborative Relationships 126

Professional Relationships $\quad 129$

Coordinator 130

Trusting Relationships 131 
Program

Strengths

Financial resources

Weaknesses

Participant Recommendations

Financial

Expansion

University Support

Goals and Mission Statement

V. DISCUSSION, CONCLUSIONS, AND SUGGESTIONS

Theoretical Framework

Personal Perspective 


\section{TABLES}

$\begin{array}{lll}\text { Table } & \text { Page }\end{array}$

1. Field-Based Program Comparisons: 1997 and 2014

2. Field-Based Participants' Characteristics 73

3. Interview Timeline of Data Collection Process 81

4. Themes, Sub-themes, and Significant Details 99 


\section{FIGURES}

Figure $\quad$ Page

1. Conceptual Framework 3

2. Field-Based Teacher Preparation Comparison Chart 4

3. Literature Map and Focus of Inquiry 48

4. Kvale's Seven Stage Interview Process Used in the Data Collection Process 76

5. Seidman's Three Stage Interview Process Used in the Data Collection Process 78

6. Kvale's Seven Stage and Seidman's Three Stage Integrated Interview Process 79 


\section{CHAPTER I}

\section{THE PROBLEM AND ITS BACKGROUND}

In some cases and in some locations, the term "field-based" is often used synonymously with the following terms: "teacher certification," "alternative certification," "teacher efficacy," "teacher preparedness," "teacher mentoring," and "teacher preparation programs." Of these terms, "field-based programs" have been the central focus of educational reform initiatives in the United States. Since the development of Normal Schools and apprenticeship programs, field-based programs have always been a topic of discussion. However, some may argue that one of the goals of field experiences includes linking theory to practice through such programs that allows participants to develop knowledge and skills through professional activities and self-reflect on their learning (Allsopp, DeMarie, Alvarez-McHatton, \& Doone, 2006; Barkesdale-Ladd \& Rose, 1997; Korthagen, Loughran, \& Russell, 2006; Rock \& Levin, 2002).

As the field of education changes, so do the expectations, leadership models, theories, and teacher development (Anhorn, 2008; Ingersoll, 2004; Melnick \& Meister, 2008). Knowing that teacher preparation expectations and standards are high, field experiences should be the most important component of teacher preparation (DarlingHammond, Wei, \& Johnson, 2009; Nahal, 2010; Wilson, 2011). Although field-based programs are unique to the university, it appears that universities share a common goal of providing teachers with well-rounded, authentic field experiences while participating in 
their programs. This study was designed to explore and interpret the experiences of graduates from an inner-city field-based teacher preparation program (INC/FBTP) to determine their perceptions of the program. Specifically, this study sought to investigate if graduates viewed themselves as prepared for the teaching profession after participation in a field-based teacher preparation program.

\section{Background of the Study}

This chapter includes a discussion of the issues, concerns, and challenges of fieldbased teacher preparation programs. Throughout this chapter, the terms interpretivism and field-based teacher preparation (FB/TPP) are used. The research presented lends itself to the theoretical foundation of interpretivism; inherent in this discussion is social and critical interpretivism and how it is connected to the field-based teacher preparation. Figure 1 illustrates the conceptual framework and the overarching questions that guided this investigation.

Many similarities are evident between field-based teacher preparation programs and professional development schools; however, this research focus was on an inner city field-based teacher preparation program. Figure 2 illustrates a comparison of this inner city field-based teacher preparation program that was adapted and modified for this research from Westbrooks and Alea (2007), the Penn State University-State College Area School District Professional Development School Partnerships (2014) and the inner city traditional student teacher program. 


\section{Interpretivism:}

What theory(s) of interpretivism are best suited to enable understanding of experiences and perceptions of participants from an inner city teacher preparation program? What connections to education and field-based teacher preparation need to be made to create meaning?

\section{Social \& Critical Interpretivism:}

How does social \& critical interpretivism inform interactions with participants in this study to derive meaning? How does social and critical interpretivism differ from other concepts that demonstrate interpretivism in the form of dialogue?

\section{Field-based Teacher Preparation}

How can we integrate social and critical interpretive theories with field-based teacher preparation to inform policy or future programs? How does field-based teacher preparation validate the construct of social and critical interpretivism in the field of education?

Figure 1. Conceptual Framework 


\begin{tabular}{|c|c|c|c|}
\hline $\begin{array}{c}\text { Program } \\
\text { Characteristics }\end{array}$ & $\begin{array}{l}\text { Field-Based Teacher Preparation } \\
\text { Programs (at the Inner City } \\
\text { University) }\end{array}$ & $\begin{array}{l}\text { Professional Development } \\
\text { Schools } \\
\text { The National Council for } \\
\text { Accreditation of Teacher } \\
\text { Education (NCATE, 2014) }\end{array}$ & $\begin{array}{l}\text { Traditional } \\
\text { student teaching } \\
\text { (at the Inner City } \\
\text { University) }\end{array}$ \\
\hline Definition & $\begin{array}{l}\text { An intensive 2-yr. program where } \\
\text { learning to teach is accomplished } \\
\text { through a collaborative partnership } \\
\text { with K-12 schools, teaming with } \\
\text { mentor teachers, university-based } \\
\text { professors, \& coordinator. }\end{array}$ & $\begin{array}{l}\text { Innovative institutions } \\
\text { formed through partnerships } \\
\text { between professional } \\
\text { education programs and } \mathrm{P}-12 \\
\text { schools }\end{array}$ & $\begin{array}{l}\text { A closely } \\
\text { structured and } \\
\text { supervised } \\
\text { classroom teaching } \\
\text { experience. }\end{array}$ \\
\hline $\begin{array}{l}\text { Admittance to } \\
\text { College of } \\
\text { Education }\end{array}$ & $\begin{array}{l}\text { Full-Time College of Education } \\
\text { Student, with a minimum } 3.0 \text { G.P.A. } \\
\text { (Not all programs) }\end{array}$ & $\begin{array}{l}\text { Full-time College of } \\
\text { Education, with minimum } 3.0 \\
\text { GPA (not all programs) }\end{array}$ & $\begin{array}{l}\text { After successful } \\
\text { completion of } \\
\text { methods courses }\end{array}$ \\
\hline $\begin{array}{l}\text { Application } \\
\text { Submission }\end{array}$ & $\begin{array}{l}\text { On-line and paper submission, } \\
\text { student interview, group challenge, } \\
\text { written essay, personal \& academic } \\
\text { references (3) }\end{array}$ & Paper application and on-line & On-line \\
\hline $\begin{array}{l}\text { Program Focus } \\
\text { Mission }\end{array}$ & $\begin{array}{l}\text { - Teacher technology training } \\
\text { - Pre-service teaching experience } \\
\text { and classroom management } \\
\text { - Assigned student to student mentor } \\
\text { - Certification preparation } \\
\text { - Research training } \\
\text { - Education conference participation } \\
\text { - Study abroad opportunities (urban, } \\
\text { rural, international) } \\
\text { - Stipend disbursements (at the end } \\
\text { of fall \& spring semesters) }\end{array}$ & $\begin{array}{l}\text { - Preparation new teachers, } \\
\text { - Faculty development, } \\
\text { - Inquiry directed at the } \\
\text { improvement of practice, } \\
\text { and enhanced student } \\
\text { achievement. }\end{array}$ & $\begin{array}{l}\text { Provide an } \\
\text { opportunity for } \\
\text { candidates to apply } \\
\text { educational theory } \\
\text { in an off-campus } \\
\text { classroom } \\
\text { setting. }\end{array}$ \\
\hline $\begin{array}{l}\text { Methods Courses } \\
\text { completed at the } \\
\text { university }\end{array}$ & $\begin{array}{l}\text { No } \\
\text { Method course are taught by } \\
\text { university field-based professors at } \\
\text { assigned field-based school. }\end{array}$ & Yes & Yes \\
\hline $\begin{array}{l}\text { Methods Courses } \\
\text { completed at } \\
\text { assigned school }\end{array}$ & Yes & No & No \\
\hline $\begin{array}{l}\text { Professors travel to } \\
\text { assigned school }\end{array}$ & Yes & Yes & Yes \\
\hline Length of program & 2 years & 1 semester to 1 year & $\begin{array}{c}16 \text { weeks } \\
\text { (200 clock hours) }\end{array}$ \\
\hline $\begin{array}{l}\text { Pre student } \\
\text { teaching } \\
\text { prerequisite }\end{array}$ & Yes & No & 100 clock hours \\
\hline $\begin{array}{l}\text { Student teaching } \\
\text { after completion of } \\
\text { the program }\end{array}$ & Yes & No, part of PDS experience & Yes \\
\hline Portfolio required & Yes & $\begin{array}{l}\text { Dependent on university \& } \\
\text { state requirements }\end{array}$ & No \\
\hline Observed lessons & 10 & $\begin{array}{l}\text { Dependent on university \& } \\
\text { state requirements }\end{array}$ & 2 \\
\hline $\begin{array}{l}\text { Community service } \\
\text { requirement }\end{array}$ & Yes & No & No \\
\hline $\begin{array}{l}\text { Stipend provided } \\
\text { by university to } \\
\text { participants }\end{array}$ & Only for teaching supplies & No & No \\
\hline
\end{tabular}

Figure 2. Field-Based Teacher Preparation Comparison Chart 
During the mid-1990s, the first inner-city field-based teacher preparation program began at a state university in an urban community. The program was first titled "FieldBased Teacher Preparation Program." Over the past 10 years, the program has made a number of changes, which include a program name change, now referred as "Field-Based Teacher Internship Program," the implementation of community service and volunteerism as part of the curriculum, and additional elementary school and high school placement sites. A field-based teacher preparation program is a collaborative partnership between the university and several selected inner city elementary and high schools. Westbrooks and Alea (2007) provide an overview of field-based teacher preparation programs. Field-based programs are designed to prepare teacher candidates for the teaching profession. Prerequisite for the program include the completion and submission of the following before admittance to the field-based program: personal interview, written essay, portfolio submission, and personal and academic references. Only students who are accepted into the College of Education's traditional program are eligible to apply to the INC/FBTP. Field-based students are required to participate in an intensive field-based practicum for a minimum of two semesters (or one year) prior to student teaching (Westbrooks, \& Alea, 2007).

During the initial stages of the field-based teacher preparation, the program focused on early childhood, elementary, and secondary majors. This INC/FBTP also incorporated an international studies program as an optional component of the program. Field-based participants were placed in a self-contained classroom with a supervising teacher for approximately 100 hours per semester, 5 days a week for 2 years. 
Additionally, participants engaged in weekly professional development workshops in an assigned school, taught the minimum of 10 lessons per semester, and met at the assigned school for reflection and collaboration on Fridays during the school hours. All participants maintained journals while participating in the INC/FBT. On Fridays, fieldbased participants were afforded the opportunity to reflect on their weekly classroom experience at the completion of the program. Additionally, participants were expected to complete student teaching and successfully pass the State of Illinois teacher certification exam.

\section{Statement of the Problem}

Teacher preparation programs have been the target of criticism for a long time. Such criticism, as outlined by the Education Commission of the States (ECS, 2008), argues that teacher education programs fail to prepare graduates with "real world" practical experience because their focus is on lenient pedagogical knowledge. The quality of preparation participants receive is directly associated with the knowledge, intelligence, and professional skills fostered during the field experience. Successful field experience is the responsibility of the mentor teacher assigned to the teacher candidate, the culture of the school, and support from the professors of the program.

There is a gap in research on the experiences and perception of graduates from teacher preparation programs focusing on classroom pedagogy, subject-matter knowledge, experience, and a combined set of qualifications measured by teacher licensure (Darling-Hammond, 2006). As a result, teacher preparation programs continue to grow based on the call to federal and state mandates such as the No Child Left Behind 
Act (NCLB, 2002) and the national standards. A growing body of researchers argue that teacher preparation programs fail to assure the quality of teacher graduates and, overall, produce inadequately prepared teachers (Anhorn, 2008; Beck, Kosnik, \& Roswell, 2007; Floden, Ferrini-Mundy, Melnick \& Meister, 2008; Nahal, 2010; Rubenstein, 2008; Salvato, 2005; Wilson, 2011). Dating back through the history of teacher preparation, assessment of the success of programs was based on teacher candidate performance observations, written exams, and state assessment data. Research of this nature has been the determining factor to assess the success of such programs. Policymakers and teacher education reformers analyzed the success rates of teachers remaining in the profession after the completion of such programs. The data was completed by teacher candidates, and the number of teachers who successfully passed the qualifying exams to determine if programs were effectively preparing candidates for the profession or if such programs were successful (Anhorn, 2008; Beck, Kosnik, \& Rowswell, 2007; Melnick \& Meister, 2008; Nahal, 2010; Rubenstein, 2008; Salvato, 2005; Wilson, 2011). Field-based research dates back to 1975 , which implies that there have been many discussions regarding how teachers are being prepared and questions that ask if field-based programs are necessary or valuable in preparing teachers (Zeichner, 2010). Research revealed that many of our schools are not preparing teachers for the teaching profession (Arbaugh et al., 2013; Darling Hammond, 2012) and insinuates that such programs provide a wealth of learning experiences and challenges for field-based participants and field-based professors.

Despite the many discussions on teacher preparation programs, a growing body of research exists on how field-based teacher preparation programs should be structured and 
designed to address the diverse needs in the classroom. Research is needed to validate the preparedness of candidates of field-based programs. In order to prepare "highly qualified" field-based teachers for the classroom effectively, participants' perceptions of the preparation received in the program need to be examined.

Education reform organizations, such as the National Council for Accreditation of Teacher Education (NCATE), Peabody reports on $20^{\text {th }}$-Century Education Reform Milestones (2014), New Commission on the Skills of the American Workforce (2009), the National Center on Education and the Economy (2014), and state boards of education, have made many recommendations on how to improve classroom pedagogy, how to implement student teaching, or how to implement field experience. NCATE (2008) standard 3, recommends that "the unit and its school partners design, implement, and evaluate field experiences and clinical practice so that teacher candidates and other school professionals develop and demonstrate the knowledge, skills, and professional dispositions necessary to help all students learn" (p. 29). However, none of the aforementioned programs tap into the experiences and perceptions of participants to assess whether teachers are prepared for the teaching profession.

The field-based teacher preparation phenomenon deserves investigation to reveal how participants perceive their experience in order to ascertain how universities can better prepare future candidates for the teaching profession. Learning from the voices of the graduates themselves is highly important because of the firsthand knowledge it offers on how the participants perceive their preparation. Hence, this research will explore this phenomenon through the theoretical lenses of interpretivism. 


\section{Nature and Purpose of the Study}

An interpretive phenomenological research paradigm was employed to explore and interpret the experiences of graduates from an inner-city field-based teacher preparation program and to determine whether they viewed themselves as well prepared for the teaching profession as a result of their participation in the INC/FBTP. Current reform initiatives focus on the need to develop teachers who are prepared for the teaching profession and who are "highly qualified" through alternative certification or field-based programs. Field-based programs have become an intricate aspect of many teacher preparation programs as a means of addressing the challenge of preparing "highlyqualified" teachers for the teaching profession. In order to prepare "highly qualified" field-based teachers for the classroom, there is a need to engage in dialogue with participants about their experiences and perceptions concerning the preparation received as participants in the program (Anhorn, 2008; Beck, Kosnik, \& Rowswell, 2007; Melnick \& Meister, 2008; Saldana, 2001; Spradley, 1979). Hence, this study focused on the perceptions of graduates who participated in a field-based program; the central phenomenon was limited to graduates from an INC/FBTP who were employed in the teaching profession at the time of this study.

In present-day society, a decision to return to a restaurant, department store, hospital, dentist, or school is based solely on personal experience or the experience of others. Many restaurants and department stores use the customer experience as a tool to guide training and to enhance a customer's experience, in order to encourage repeat business. Understanding the perceptions of graduate experiences in a field-based teacher 
preparation program offers important implications for raising the bar in teacher preparation and promotes a need for longer authentic field-experiences for future graduates (Darling-Hammond, 2013). Just as the experiences of customers inform many businesses nationwide, hearing the experiences of graduates from a field-based teacher preparation program can inform policymakers, university teacher educators, and principals on how to improve field experience for future teachers.

The purpose of this interpretive study was to explore and interpret the experiences of graduates from an inner-city field-based teacher preparation program to determine their perceptions about their preparation. Specifically, this research provides clear and concise findings on the unique experiences and perceptions of the participants in the following areas: perception of preparedness; knowledge, skills, and attitudes that are critical for success; and relationships and experiences with professors and mentor teachers. More importantly, documentation of the specific experiences, as perceived by field-based participants guides future programs to gain knowledge on how the data contributes to the success of future programs and the development of teachers for the teaching profession.

An attempt to understand what meanings of field experiences have for graduates, and how the field experience prepared the graduates for the teaching profession, underscored this inquiry. It is important to understand field-based teacher preparation, because Secretary of Education Duncan (2009), Darling-Hammond (2011), NCATE, and state boards of education are requesting the implementation of field-based programs and longer field experience nationwide. Field-based teacher preparation is a growing 
phenomenon in education that others insinuates can provide teachers with an opportunity to connect theory to practice more effectively (Beeth \& Adanan, 2006; DarlingHammond, 2011; Duncan, 2009; Nahal, 2010; NCATE, 2010; Tigechelaar \& Korthagen, 2004; Wilson, 2011).

Although, interpretivist research has been viewed from the construct of analyzing written text, for the purpose of this study, interpretivism is introduced as a theoretical framework to elucidate the experiences of the participants and enable oral dialogue about the effectiveness of future field-based teacher preparation programs. Most importantly, interpretivism is defined in the context of oral dialogue from the field-based participants to identify needs and gaps and to make recommendations for the implementation of future field-based teacher preparation programs.

\section{Research Question}

The researcher explored and interpreted the experiences of graduates from an inner-city field-based teacher preparation program (INC/FTP) to determine their perspective. This research investigated participants' views of themselves as well prepared for the teaching profession due to participation in a field-based teacher preparation program. Specifically, the researcher wished to answer the overarching question: What are the perceptions and experiences of participants in an INC/FBTP, in terms of: (a) perception of preparedness; (b) knowledge, skills, and attitudes that are critical for success; and (c) relationships and experiences with professors and mentor teachers. It is essential to understand the experiences and perceptions of field-based participants in order to determine if field-based teacher preparation programs adequately prepare 
teachers. Research proposes that field-based teacher education programs are in line with many current teacher education programs based in a positivist tradition, where discrete courses are offered, fragmented between departments, and with little or no connection to field-based experiences (Anhorn, 2008; Beck, Kosnik, \& Rowswell, 2007; Melnick \& Meister, 2008; Nahal, 2010, Darling-Hammond, 2008; Rubenstein, 2008; Salvato, 2005; Wilson, 2011).

\section{Significance of the Study}

In 1956, a study was conducted on field experiences. This study identified that a gap existed in research that validated that longer field experience is needed (Zeichner, 1981). Joyce et al. (1977) argued that field-experience was a crucial component of teacher preparation. This study adds to the growing body of scholarly literature on the experiences and perceptions of participants who participated in field-based teacher preparation programs in the wider educational community. Current research on fieldbased experiences posits that many factors influence teacher candidates and their participation in such programs; participants' perceptions of the curriculum, experiences, and personal and professional preparation differed among the participants in field-based programs. Additionally, participants developed self-efficacy, gained a higher level of respect for other cultures, and valued feedback and reflection (Bergman, 2013; Duquette, 1997; Joekel, 2010; Pence, 2008; Washburn-Moses, Kopp, Hettersimer, 2012). Others argue that there is a lack of evidence that field-based programs adequately prepare teachers for the teaching profession or that longer field experience prepare teacher candidates for the teaching profession (Anderson \& Stillman, 2013; Darling-Hammond, 
2010; Guyton \& Byrd, 1999; Perry, 2011). The inadequate research on the experiences and perceptions of graduates in field-based teacher preparation programs lends itself to the social constructs of teachers not being prepared for the teaching profession and the need for a longer field experience in teacher preparation.

The results of this research will be useful to policymakers, state boards of education, and universities and colleges of education by identifying key themes derived from the participants' interviews and using the data as a tool to inform key stakeholders in the wider education community. Additionally, the study attempts to provide a framework to improve teacher preparation field experiences as a tool to guide education policy. Darling-Hammond (2007) explains that changing past practices in teacher preparation in relation to field-based experiences could prepare teachers for the academic classroom challenges to become the "highest quality of teacher" (Darling-Hammond, 2007, p. 347).

Universities, policymakers, and school administrators can utilize the themes that evolve from the interviews as a tool to identify whether teachers are making connections with students, learning how to create collaborative lessons, or benefiting from an improvement in the overall field experience that focuses on collaborative lesson planning. Most importantly, it improves and strengthens field-based programs and partnerships with collaborating schools and gauges the preparation of teachers for future employment.

Finally, this study may improve policy if colleges and universities take an indepth look at the experiences of the field-based participants-to provide more effective authentic field experiences in teacher preparation programs nationwide. As an 
interpretative research study, this research is the fulcrum that transforms field experiences to inform, support, or challenge policy and educational reform efforts. Next, the wider education community can learn how to support future field-based teacher candidates in the program and address their needs. Policymakers can gain access to firsthand knowledge from the participants' experiences on how the field-based experience has prepared them for the teaching profession and which areas the graduates identify as needing additional development. Articulating the value of field-based experiences of graduates can provide recommendations and enhance future field-based programs nationwide.

\section{Theoretical Foundations}

For many years, the evaluation of teacher preparation was based on assessment data, surveys, or performance assessment of the student teaching experience. Because this research study explored field-based teacher preparation in the context of participant experiences, theory about interpretivism is outlined. The theoretical foundation of this qualitative study integrates social theory and critical interpretivism, which includes language, culture, and experience. From the interpretivist perspective, interpretivism infers or decodes the individual perspective, character, or behavior from the individual's perspective. Interpretivism is a philosophical approach that studies the experience through text, "making sense of what is happening to the participants"' (Smith, Flowers, \& Larkin, 2009, p. 3). Interpretivism, as an educational theoretical approach, lends itself to exploring the experiences and perceptions of participants from a field-based teacher preparation program. Smith, Flowers, and Larkin (2010) maintain that there are four leading authorities on the theory of interpretivism: Schleiermacher (1998), Heidegger 
(1962/1927), Gadamer (1990/1960), and Kvale (1996). Schleiermacher (1998) clarifies interpretivism as a grammatical and psychological interpretation, which is concerned with the individuality of the speaker and identified as a craft that required the use of several skills and intuition in order to understand the writer and text. Schleiermarcher also proposes that interpretivism evolves during the engagement of text analysis, dialogue, or associations.

A review of literature proposes that interpretivism is a form of hermeneutics that provides for interpretation of text to obtain a valid and common understanding of meaning. (Kvale, 1996; Smith et al., 2010). For many years, the word "text" in classical hermeneutics entailed literature, religion, law, discourse, and action. According to previous research, hermeneutics can be used as a tool to interpret "literary works" in an attempt to show relationships and meaning of the text (Kvale, 1996; Smith et al., 2010).

Heidegger (1962/1927) argues that previous works of interpretivism focused with the "exact" and objective textual meaning, whereas his works focused on the "individuality of the author or speaker" (p. 22). Between 1990 and 1996, Gadamer emerged with the concept of analyzing historical and literary text and the effects of tradition on the interpretive process (Gadamer, 1990/1996; Kvale, 1996). Gadamer points out that as the researcher begins to understand the preconceptions, the interpretations are in process. Kvale (1996) implies that the "thing" itself influences the interpretations.

This inquiry introduces interpretivism in the form of interpreting the experiences and perceptions from the graduates' perspectives, because there is inadequate research on 
experiences of participants as told orally. It is essential that this interpretive approach explore how the key elements of interpretivism are related to the field of education in order to interpret and present the experiences of graduates from an inner-city field-based teacher preparation program. Smith et al. (2010) provides many versions of interpretivism. After a critical reading of these theories, an interpretive theory postulates that an interpretation of our daily experiences or the experiences of others enables understandings of what is real in the world and interprets the experiences of graduates for this research. This position is guided by Gadamer's (1990/1960) Being and Time theory of interpretivism and grounded in the works of Smith et al., (2010) Schleiermacher (1998), and Gadamer (1990/1960).

Interpretivism as a theoretical foundation provides a valid and common understanding of meaning that is interdependent and mutually interactive. It enables a collaborative construct of perceived reality. This theory implies that knowledge can be adapted to new situations, and that knowledge of social and cultural realities can be negotiated (Gadamer, 1990/1960; Kvale, 1996; Smith, Flowers, \& Larkin, 2009).

Social interpretivism enables the understanding of how the social world influences experiences, which in turn is collaborative between or among people. (McLaren, 2007; Rowlands, 2005; Schwandt, 1997). Social theory is used to study student teachers, teacher education programs, and pre-service teachers (Schoeman \& Mabunda, 2012). Hence, social interpretivism was used in this study to examine the perceptions of graduates from an INC/FBTPP. Social interpretivism is an intricate aspect of this research because it seeks to understand the various program components and 
concepts that enable the researcher to explore how the field-based programs prepare teachers for the teaching profession (Dean, 1996; Flores, 2007; Denzin, Norman, \& Lincoln, 2012; Schwandt, 2007; Westbrooks \& Alea, 2007). With this in mind, it is necessary to capture the essence of the participants' experiences during their field-based teacher preparation program (Dean, 1996; Denzin \& Flores, 2007; Norman \& Lincoln, 2012; Schwandt, 2007; Westbrooks \& Alea, 2007).

Critical interpretivism (CI) is closely linked to forms of control, issues of justice, and equality in relation to the process of collecting and interpreting data (Freire, 2005; Giroux, 2011; Hinchey, 1998; Wink, 2005). CI enables researchers to use, adapt, and apply concepts to deepen their understanding of social and field experience to develop as agents of change. For this purpose, critical interpretivism is presented to comprehend research in teacher education, especially as it relates to teacher preparation programs. Williams (2012) argues that critical interpretivism "encourages social cultural transformation through inquiry and analysis to issues of power, response to justice and equality among others."

The term sociocritical interpretivism (SCI) is introduced as an integration of two theoretical frameworks that is applied to field-based teacher preparation programs, which questions concerns and issues of power and equity and discourse in field-based teacher preparation programs. SCI provides the opportunity to explore the role of criticism to determine if field-based teacher preparation programs provide quality education. Leonardo (2004) argues that quality is proportioned to the depth of analysis that fieldbased participants acquire during their participation in the field-based teacher preparation 
program. SCI explores the program discourse analysis and the participants' ability to analyze the field-based teacher preparation experiences that result in empowerment. Additionally, SCI focuses heavily on values and progress developed among the participants and closely analyzes the ideologies of power within the field-based teacher preparation program. The goals of this research study thus fell directly in line with this theory.

The combination of the these theories provided context in this study by providing an explanation of the experiences and perceptions of participants in an inner city fieldbased teacher preparation program (INC/FBTPP) as it related to their development as professionals. Further detail regarding interpretivism and the more specific sociocritical interpretivism are provided in the literature review.

\section{Definition of Terms}

For the purpose of this study, the following definitions were applied to assist with clarity of understanding. The terms are listed in alphabetical order. The terms preceded by an asterisk denote a terminology that was developed by the researcher.

*Critical interpretivism: use, adapt, and apply concepts to deepen understanding of critical and social field experience to develop as agents of change. It facilitates a process in which the researcher learns to interpret the experiences in the field as told by others.

Field-based: a program that supports or serves as a foundation to prepare teacher candidates for the professional field. 
Field experience: students completing observations, pre-clinical hours, practicum experiences, and pre-student teaching hours. A variety of early and ongoing field-based opportunities in which candidates may observe, assist, tutor, instruct, and/or conduct research (NCATE, 2007, p. 54).

Field-Based Teacher Preparation Program (FB/TPP): School experiences where teacher candidates are afforded the opportunity to observe, immerse themselves in the school, and become acclimated to the culture of the school (Westbrooks \& Alea, 2007; MacDonald et al., 1992; Duquette, 1993; Shen, 1994; Wiseman, 1995; Sanford \& Hopper, 2002).

Highly qualified: The rigorous qualifications of candidates at admission, exit, and throughout a preparation program as judged through selective criteria on a recurring basis by EPPs (CAEP, 2014).

Interpretivism: a form of hermeneutics that provides for interpretation of text to obtain a valid and common understanding of meaning. (Kvale, 1996; Smith et al., 2010).

Professional Development Schools (PDS): Specially structured schools in which the P-12 school and higher education faculty collaborate to (a) provide practicum, student teaching, and internship experiences; (b) support and enable the professional development of school and higher education faculty; (c) support and enable inquiry directed at the improvement of practice; and (d) support and enhance student achievement (National Professional Development Schools p. 1).

*Social critical interpretivism: understanding of how the social world and power influences experiences, and how participants transform their professional practices. 
Gaining a clear and precise focus of discourse and the central role equity, and power play in understanding the many issues involved in the social constructs of education, and fieldbased teacher preparation programs and the role of language, culture, and experience in the context of field-based teacher preparation programs.

*Social interpretivism: gaining an in-depth understanding of how the social constructs influences education and its role in education and learning and participants' experiences.

Student teaching: an intensive and extensive culminating classroom experience, which includes a set of responsibilities, assignments, tasks, activities, and assessments that demonstrate candidates' on-going development of the knowledge, skills, and dispositions to be effective educator.

\section{Conclusion}

In this chapter, field-based teacher preparation programs were defined and described. In addition, interpretivism and the development of social and critical interpretivism were described relating them to the field of education and field-based teacher-preparation programs. Moreover, the combination of the two theories was explained to provide context in this study and the experiences and perceptions of participants in an inner city field-based teacher preparation program (INC/FBTPP) as it related to their development as professionals.

The development of sociocritical interpretivism was described to articulate how it relates to the field of education and field-based teacher-preparation programs. Interpretivism in field-based teacher-preparation programs provides a foundation for a 
philosophical approach to teacher preparation and field experiences in which the understanding of field experience is given high regard. An interpretation of the experiences and perceptions provides a foundation for determining the general goals for future field-based teacher preparation programs, traditional teacher preparation programs, and student teaching. Additionally, this chapter outlined how interpretivism assisted in the understanding of how to explore and interpret the experiences of graduates from an inner-city field-based teacher-preparation program to determine how they felt about the preparation program. Through the understanding of interpretive concepts as truth, meaning, and work experience, the researcher was able to gain a better understanding of how graduates view themselves prepared for the teaching profession as a result of program participation. 


\section{CHAPTER II}

\section{LITERATURE REVIEW}

Traditionally, literature reviews consist of one specific area of research. For the purpose of this study, the literature review contains two topics of research: interpretivism and field-based teacher preparation programs. The integration of these two research areas is critical because it demonstrates how interpretivism is connected to education. Interpretivism has always been used as a methodology. In this research, interpretivism is discussed in the literature view because it introduces social interpretivism, critical interpretivism, and interpretivism as they relate to education and interpretivism as it relates to field-based teacher preparation. The discussion of interpretivism is used to set the foundation of for understanding the theory of sociocritical interpretivism.

Field-based teacher preparation programs are a growing phenomenon, not only in the United States but in Great Britain and Canada, as a strategy to improve teacher preparation (Duquette, 1997). Educational research studies have explored field-based teacher preparation programs, but a need exists for more educational research that explores the perceptions of participants from a field-based teacher preparation program. Wright (2009) implies existing research on field-based teacher preparation has failed to analyze the perceptions of how field-based experiences have prepared teachers for the 
teaching profession. Conversely, Moore (2008) argues that field-based teacher preparation programs have been identified as a critical means of the redesign for teacher preparation. Previous research advocates a need for improved field-experience that is focused on a program that connects both public school and university experiences (Holmes Group, 1986, 1990). Some argue that field-based programs have been identified as a critical means for redesign for teacher preparation (Metcalf, 2014; DarlingHammond, 2014).

\section{Field-Based Defined}

According to Webster's On-line Dictionary (2014), the word "field" originated from the Old English word "field," which had several meanings. In relation to this study, "field" is an area or division of an activity, subject, or profession, of practical operation outside a base (as a laboratory, office, or factory). On the other hand, the origin of the word "base" derived from the Latin term "basis," which has two meanings that relate to this research: (a) the bottom of something considered as its support, and (b) the fundamental part of something, the groundwork, and basis. Hence, "field-based" is a foundational program that supports or serves as a foundation to prepare teacher candidates for the professional field. In the field of education, the researcher, teachers, and employers use interviews as a means to gather data, to give voice to the participants, and to engage in dialogue to become familiar with the participants.

There is a growing awareness of field-based teacher preparation at colleges and universities, which provides participants with opportunities to acquire skills and dispositions necessary for the teaching profession. It is equally important to attempt to 
better articulate the nature and the extent of such programs as they relate to the field of education (Anderson \& Stillman, 2013; Capraro, Capraro, \& Helfeldt, 2010; DarlingHammond, 2012; Rose, 2013). In order to decipher the perception of the participant, which helps in recreating field-based participants' individuality in the social and cultural context, it was important to explore the following as they relate to the inner city fieldbased teacher preparation program (INFB/TPP): (a) prior experience; (b) time spent in the field; and (c) language and culture in the school community.

\section{Prior Experience}

Field-based teacher preparation (FB/TPP) is not a new phenomenon in the field of education. The question still remains: Are field-based teacher preparation programs preparing participants better for the teaching profession (Adams et al., 2005; Cristol \& Gimbert, 2002; Zeichner \& Melnick, 1996). These FB/TPPs consist of a wide range of students from recent high-school graduates to mature adults with prior work experience from other fields. Field-based participants bring a wealth of experiences to the program that adds to the overall learning experience. Current research posits that prior experience should not focus solely on classroom experience but could extend to curriculum planning, professional development, working with the community or parent groups, etc. However, it does not exclude prior classroom experiences, such as student teaching, substitute teaching, shadowing, or completing an internship. Prior experiences are brought to the FB/TPP to help reconstruct the individual experiences into meaningful insights into the teaching profession. Prior work experiences enable participants to make a connection with self-knowledge to theory where some believe that prior experience is the 
determining factor when making personnel decisions regarding seniority and inequity (Rice, 2010). Prior experience provides instruction to the direction of how to provide a conducive and productive learning experience that addresses learning needs of each student and the needs of the school.

\section{Field-Experience}

The field of teaching is a far more multifaceted profession than field-based teacher participants realize (Cookson, 2005), and amount of time field-based participants spend in the field is an essential aspect of the FBTPP. In the past, many Colleges of Education followed the NCATE Accreditation Resource Guidebook (2007), which defined field experience as a "variety of early and ongoing field-based opportunities in which candidates may observe, assist, tutor, instruct, and/or conduct research" ( NCATE, 2007, p.54). Field-experience is designed to offer teacher candidates an opportunity to gain hands-on experience and insight of the teaching profession in the classroom and to cultivate the teaching skills necessary to become effective teachers. Field-based teacher participants who begin in the classroom on the first day of the academic school year learn how the realities faced in the classroom can be an eye-awaking experience for everyone (Melnick \& Meister, 2008; Murshidi, Konting, Elias, \& Fooi, 2006; Nahal, 2010). Huling (1999) evokes that field experience is similar to the experiences of medical students who participate in internships and residencies, in that participants observe, and work with real students, teachers, and curriculum in natural settings (Huling, 1999).

Field-based experience requires participants to interpret and apply to the teaching profession what was taught and learned at the university. Field-based participants learn 
skills required for teacher certification and are introduced to the profession of teaching. This early stage involves visits to schools and classrooms to observe the school climate and the process of teaching to gain an in-depth knowledge of the role of the schools, classrooms, and teachers. The field experience placement is contingent upon the availability of the school and the academic progress of the students in the school. The placement may be in a public or private school, an elementary, middle or high school. In some cases, students are afforded the opportunity to travel abroad to further their fieldbased teacher preparation experience (Westbrooks \& Alea, 2006).

\section{Historical Perspective}

According to history, the term "field-based" began with the medical profession's concept of residency or clinical experience, which medical students were required to complete prior to becoming medical practitioners. According to Huling (1998), field experience was grounded in the work of John Dewey $(1904 ; 1935)$. Dewey advocated for stronger experiential teacher training and experiences that provided the construction of the individual learning (Hauling, 1998). Sanford and Hopper (2002) state that initial field-based teacher preparation refers to school experiences where teacher candidates are afforded the opportunity to observe, immerse themselves in the school, and become acclimated to the culture of the school (Duquette, 1993; MacDonald, 1992; Sanford \& Hopper, 2002; Shen, 1994; Westbrooks \& Alea, 2007; Wiseman, 1995). During the 1970s, field-based was termed "professional development complexes," which "existed only in England, Wales, Holland, and Japan" (Harty, 1978, p. 340). These programs provided ongoing hands-on field experiences and resources that were developed 
collaboratively by teachers (Harty, 1978). Field experience occurs in educational settings, such as laboratory schools, professional development schools, public schools, and private schools.

During the mid-1990s, the term "field-based teacher preparation" resurfaced and one inner-city university addressed the demand for a longer and authentic field experience by implementing the first Field-Based Teacher Preparation Program. In this FBTPP, teacher candidates were housed in different elementary schools and high schools each semester in surrounding neighborhoods of the university in order to provide an authentic experience in a school community. Exposure to the teaching profession early in the educational career allowed principals to groom these future teachers for future employment. Additionally, this experience allowed participants to attend all school-wide functions, learn the nuances of the being a teacher, and showed them how to complete individual educational plans, attend school-wide trainings, etc. (Darling-Hammond, 2006; Westbrooks \& Alea, 2007).

Field-based teacher preparation programs are growing nationwide. Currently, many field-based teacher preparation programs require students to withdraw from their traditional teacher preparation programs and register for the field-based program. Participants of field-based programs are expected to spend time in their assigned fieldbased schools while completing their required methods courses. Many universities, such as Northern Illinois University, the University of Texas El Paso (2014), Chicago State University, Illinois State University, University of California, Northeast Texas Center for Professional Development and Technology (2013), Texas A \& M (2013), and the 
University of Washington (2013), all have field-based teacher preparation programs. The University of California (2014) College of Education defines field-based programs as:

Field-based teacher preparation involves immersing participants in various school settings as they plan, teach, reflect, and apply. Field-based education is a provenand-tested approach to prepare teachers for the challenges of the teaching profession by linking theory into practice, moving from reflection into action, and shifting from empowered to empowering.

(http://www.csub.edu/maeducation/btsa_emph.html)

NCATE states that the importance of understanding the experiences of field-based participants is a way to assess whether field-based teacher preparation programs "better prepare" future teachers by linking theory to practice (NCATE, 2007). Field experience provides a foundation for future teachers by affording participants the opportunity to connect methods courses to the classroom with on-the-job teaching experiences (DarlingHammond, 2007; NCATE, 2007).

Across the United States, colleges of education face the demand of producing teachers who are prepared for the challenges of the classroom and the various levels of diverse learners. During the 1970s, a number of criticisms developed regarding fieldbased teacher preparation programs. Arguments imply that "field-based experience is a traditional school of thought that serves merely to socialize prospective teachers into established patterns of school practice" (Hooper \& Johnston, 1973; Friedenbury, 1973; Sanders, 1974). Criticism continued to debate the purposes of field-based programs, which promote "curricular testing," on-going testing of educational promises and the assessment of the effects of field-based programs (Salzillo \& Van Fleet, 1973). Moore (2003) argues that there is a limited amount of research that validates improved student 
performance or preparation as a result of field-based participation or experiences. Zeichner (1981) argues that it is difficult to identify a concrete conclusion from the aforementioned criticism. This research was conducted during the early developmental and implemental stages of field-based programs.

During that era, previous research on field-based programs discussed the strengths and weakness, but the participants' experiences and perceptions had not been discussed (Moore, 2003; Ross, 2001; Zeichner, 1981). However, in Ratzeoff's (1986), Perceptions of Student Teachers University Advisors, and Associate Teachers, the author proposes that there is existing research on the perceptions of student teachers or graduates. Existing research on field-based programs indicates specific programs are comprised of surveys and seminars that are unique to the field-based university. The field-based programs in Canada are taught by retired practitioners or part-time professors. Duquette (1997) identified seven previous studies on field-based teacher preparation programs prior to 1997. Specific to these studies, the research focused on coursework, field-based experiences, and professional outcomes.

Conversely, a number of qualitative researchers have affirmed the importance of perspectives of the participants and how they perceived their preparation for the teaching profession (Bogdan \& Taylor, 1982; Blumer, 1999; Mezirow, Darkenwald, \& Knox, 1975; Rock, 1989; Rockhill, 1982; Smith, 1993). Others argue that research validates the benefits of field-based programs and the supporting testimonials on such benefits. Duquette (1977) informs readers that field-based programs "have been viewed as a means to improve teacher education in Canada and Great Britain. Placing participants in 2-4 
different schools over a two-year program affords participants an opportunity to gain a wealth of hands-on diverse experiences."

According to research, the field-based program in Canada affords participants the opportunity to develop "practical skills" and opportunities to observe and work with students in the classroom; participants are given extended field experiences to gain knowledge and understanding of the culture of the school (Duquette, 1977). Ross (1995) argues that extended field-based experiences integrate the participant's experience with content knowledge and disciplined knowledge. Field-based participants gain experiences observing various classrooms, working with mentor teachers and teaching strategies; tutoring individual and groups of diverse learners; and teaching lessons to large and small groups (Capraro, Capraro, Parker, Kulm, \& Raulerson, 2005).

Urban field-experiences are critical components of field-based programs because they introduce participants to the realities of the job market, which may encourage participants to seek employment in urban districts (Burns, Grande \& Marble, 2008; Swartz, 2003; Wolf, 1996). Others contend that field-experiences in urban schools may serve as a means to decrease participants' fears and develop respect for both urban students and teachers (Heinemann, Obi, Pago, \& Weiner, 1992; Pagano, Weiner, Obi, \& Swearingen, 1995). Proctor, Renty, and Jackson (2001) validate the argument that placing field-based participants in urban neighborhood school has the potential to influence the participants' decision to apply for employment in the inner city schools. Proctor et al. proposes that participants should have the opportunity to reflect on their 
learning and beliefs about diversity in the classroom in order to understand how to confront the daily challenges in the classrooms (p. 103).

Current research regarding the length of time in various field-based programs alludes that the impact of differing field-based experiences may have positive results (Shanahan, 2008; Zeichner, 1980). A review of literature reveals that there is a limited amount of research on field-based programs, yet there is still a need for intensive field experiences that integrate reflection and inquiry, connecting current educational theories with individual learning (Capraro, Carparo, \& Helfeldt, 2010). However, a misconception still exists that "all field-experiences result in a positive experience(s)" (Zeichner, 1980).

Despite the growing demands to prepare highly qualified teachers for the nation's classrooms, a number of questions remain regarding preparing teachers for the teaching profession. According to the U.S. Department of Education (2011), there is no national definition of the term highly qualified; it is defined by each individual state. The 23 Illinois Administrative Code Section 25 defines highly qualified as "any teacher with the primary responsibility of delivering instruction in the core academic subject areas." This includes most of the main subject areas, such as math, English, science, and history. These demands are a result of many discussions about what teachers should know and be able to do at the completion of their academic program.

The issue of how to improve teacher quality and preparation has led to several discussions focusing on longer field experiences. President Obama (2009) and U.S. Secretary of Education Arne Duncan (2009) challenged the nation's universities to make 
significant advancements in teacher preparation programs. President Obama's education administration committee worked collaboratively to enhance the current teacher preparation programs through the development of Teacher Quality Partner (TQP) grants and actively listening to national agencies for teacher education reform (Duncan, 2009; Obama, 2009). U.S. Secretary of Education Duncan (2009) argues that it is not the social or cultural issues that prevent the success of students. Student success is heavily dependent upon the quality and delivery of instruction from the classroom teacher. He continues to stress that a large majority of colleges of education are performing average in preparing teachers for the teaching profession. Current reform initiatives and DarlingHammond (2006) recommend higher standards in teacher preparation programs and a minimum of "30 weeks of field-experience and student teaching opportunities in the teacher preparation program (Darling-Hammond, 2006; NCATE, 2007).

Darling-Hammond (2006) further discusses the importance and need to study the experiences of teacher candidates as well as understand how field experiences prepare future teachers for the teaching profession. She argues that more discussions are needed on the intricate aspects of field-based teacher preparation programs, curriculum, and field experiences of participants in order to assess an attainable outcome of participants in these programs. Research confirms that, at the onset of field-based programs, classroom pedagogy develops through mentor modeling, observations, and on-going collaboration (Hammond, 2006).

A review of literature reveals that although there is existing research on fieldbased teacher preparation programs, there is limited research on the experiences and 
perceptions of participants of such programs. Existing research focuses on the type and amount of curriculum that effectively prepares teachers to face diversity in the classroom, participant perceptions in literacy, and participant perception in special needs classrooms or primary classrooms (Sleeter, 2001; Webb-Johnson \& Articles, 1998). In her research titled, Conflicting Perceptions of Participants in Field-Based Teacher Education Programs, Dequette (1997) identified seven prior studies on field-based teacher preparation programs, or what she termed "on-site programs," which included both elementary and secondary levels, and participant perceptions as it related to experience, course work, and professional competencies. These research studies discuss the perceptions of participants in content-specific areas in the teaching profession. The gap in this research is the investigation of the experiences as told by the participants. These studies provide a valid argument for field-based programs and the role they play in preparing participants for the teaching profession. An interesting fact outlined in each of the studies elude to the fact that research validating longer field experiences prepares participants for the teaching profession. However, the articles do implies that field-based programs introduce participants to the nuances of the teaching profession. Figure 3 articulates research from 1997 that was similar to this study. The figure provides insight to the content of the study that was conducted in 1997 and 2014. The major differences in the 1997 study were that the research focused on three elements: curricular, experiential, and professional competency of the participants; the professors who were involved in the program and the coordinator; and the role of the students in the program. However, the 
research did not seek to understand the experiences and perceptions of the participants from such programs or investigate the experiences as told by the participants.

Table 1

Field-Based Program Comparisons: 1997 and 2014

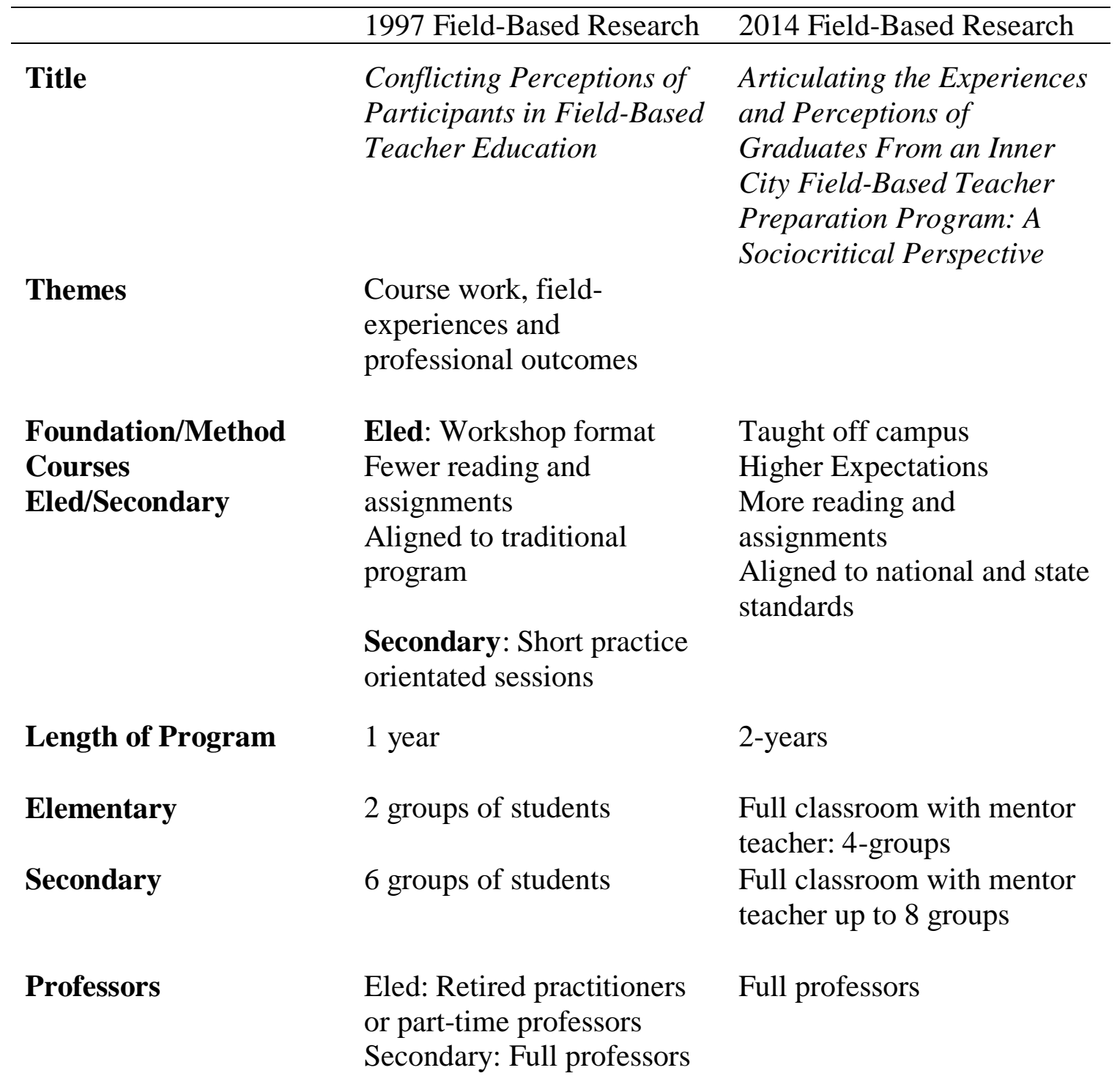




\section{Site visit or Observation(s)}

School Mentors Method courses
Elementary: Once a week/semester Secondary: Professors visit associate teachers on site every week to address concerns/issues

Note. Eled is the abbreviation for elementary education.

A limited amount of research focuses on the experiences and perceptions of graduates of field-based teacher preparation programs in terms of their preparation, the knowledge, and skills gained, and relationships developed while participating in such programs. Moore and Sampson (2008) indicate that there are a number of studies on the effects on participants who participated in field-based programs or on the social implications of participants who are placed in communities, they are most familiar. Many researchers argue a critical need that teacher candidates of field-based programs gain experience in working with children diverse backgrounds (Ladson-Billings, 2005). However, it is unclear how field-based experiences negatively or positively influence or prepare participants for the teaching profession while participating in a field-based teacher preparation programs (Perry, 2011; Rose, 2013). It is crucial to gain a clear and concise understanding of the meaning these field experiences had for the graduates. This understanding will assist researchers, policymakers, and educators in the development of curriculum, and the analysis of theories, data, and policy to enhance educational research and methodological skills and comprehension. Huling (1998) argues several principles 
indicate that candidates who were prepared in field-based programs perform similarly like second-or third-year teachers during their first year of teaching.

A review of literature on field-based teacher preparation programs reveals that after teacher candidates are accepted to Colleges of Education, candidates complete an intensive field-based practicum for a minimum of two semesters (or one year) in addition to their coursework and student teaching. Experiences include, but are not limited to, working collaboratively with a supervising or mentor teacher, team teaching, weekly reflection with peers, participation in school-wide activities, completing university methods courses in the elementary school in lieu of the university, and completion of student teaching at the completion of the program (Perry, 2011; Westbrooks \& Alea, 2007)

NCATE (2007) Standard 3 indicates performance-based field experiences are schools connected to the teacher education program that provide well-designed opportunities to learn by doing. Caprara et al. (2010) argue that many reformers and professional agencies, such as Dewey (1965), Barth (2001), the Carnegie Forum on Education (1986), and the Holmes Group (1986), all advocated for the essential role of field experience in the preparation of teachers. As a result, teaching standards are higher now than ever before, and teacher candidates are required to have a longer field experience to develop skills necessary to construct and manage diverse learners and to self-reflect on their classroom practices.

Field experience is an intricate aspect of field-based teacher preparation programs which employ the foundation for the teaching profession by affording field-based 
participants hands-on, authentic experience in classrooms of various levels and in the community (Andrews, 2009; Edwards \& Kuhlman, 2007, National Council for Accreditation of Teacher Education, 2002). NCATE recommends that teacher candidates have collaborative field-based experiences through the duration of their teacher preparation program. Collaborative field experience affords teacher candidates the opportunity to experience the teaching profession and engage in dialogue about the various challenges that teachers experience daily. Abell (2002) emphasis that the field experience transpires during the methods section of teacher preparation programs, where teacher candidates spend time in the classroom to learn from practicing teachers. NCATE Blue Ribbon Panel (2010) examined the "status of the field-experience" and found a number of inconsistencies in teacher preparation, such as the length of time a candidate spends with a mentor teacher, length of field-experience time, requirements for student teaching, or no clinical experience whatsoever. A review of literature reveals that existing research on "field experience appears to be somewhat equivocal as the learning that occurs during field experiences and appears to be highly contextualized and uneven" (Ritter, Powell, \& Hawley, 2007; Téllez, 2008, in Caprara, Caprara, \& Helfeldt, 2010). Franco (2006) adds that teacher candidates' knowledge about lesson planning and teaching are influenced by the experiences encountered in their teacher preparation courses and affect the kind of teacher they will become in the future.

\section{Catapano (2010), in Preparing Teachers for Urban Schools: Evaluation of} Community Based Model, states that field-based programs in neighborhood schools help participants make a connection with the teaching profession, pedagogy, and content 
covered in the university courses. An advantage of interpretive research is the ability to interview, which provides significant data to understand a broad range of experiences from the field. During the interpretive process, participants are encouraged to reflect on their experience to embrace the meanings and experiences brought to them. Eliciting a verbal account of experiences involves questioning, in order to reveal the perceptions of the participants. Interpretivism assists in understanding how these experiences and perceptions change as a result of longer field experience. Field-based teacher preparation programs are organized to plan, act, and reflect in order to offer participants customized courses to connect with the cultures of the children in public education (Adams et al., 2005; Andrews, 2009; Edwards \& Kuhlman, 2007; Gomez, 1996; Lenski, Crawford, Crumpler, \& Stallworth, 2005).

Field-based teacher preparation programs provide teachers with the opportunity to experience the reality of the teaching profession. Longer field experiences in schools afford principals and school districts the opportunity to groom future teachers (Glazer \& Hannafin, 2006; Adams, Bondy, \& Kuhel, 2005; Hollins \& Guzman, 2005; Leland \& Harste, 2005). Many Colleges of Education nationwide are implementing field-based teacher preparation programs to offer long field experiences and prepare teachers for the teaching profession (Duncan, 2012; Glazer \& Hannafin, 2006; Zeichner, 1996). Interpreting the perceptions of graduates from field-based teacher preparation programs and the meaning of the various roles groups deserves investigation for the implementation of future programs and preparation of participants. 
Field-based experiences involve a large number of participants, professors and mentor teachers placed in a number of collaborative schools, and the exploration of participants' perceptions of their experiences is imperative. Investigating how field-based teacher preparation program experiences negatively or positively influence participants' perception of the teaching profession plays an important role as we strive to improve teacher preparation nationwide. The on-going desire to gain a clear and concise understanding of field-based teacher preparation programs and the meaning these field experiences have for the graduates will assist researchers, policymakers, and educators in the development of curriculum and the analysis of theories, data, and policy to enhance educational research and methodological skills and comprehension.

Research on field-based teacher preparation has been conducted on teacher mentoring and alternative certification, but no research is evident on the experiences and perceptions of participants in these programs, particularly in FB/TPP. To address this gap in the literature, interviewing strategies of Kvale (1996) and Seidman (2006) and interpretive research analysis was implemented to explore the perceptions and experiences of graduates from an inner-city field-based teacher preparation program. It is critical to understand the experiences and perceptions of field-based participants in order to determine whether field-based teacher preparation programs "better prepare" future teachers for the teaching profession. Research argues that field-based teacher education programs are in line with many current teacher education programs based in a positivist tradition, in which discrete courses are offered, fragmented between departments, and with little or no connection to field-based experiences (Grimmett, 1988; McWilliam, 
1994; Zeichner 1999). Kingsley (2008) contends that the implementation of coursework, extended field experiences, and a "strong mentor teacher" provides participants the opportunities to develop their teaching philosophy.

Research between 1986 and 1996 argues that a request for significant changes in teacher preparation was needed as a foundation for educational reform efforts. Many reform organizations believed that teacher preparation should be rigorous and more authentic in order for teachers to manage the ongoing challenges in the classroom.

For many years, policymakers and teacher preparation programs have based their knowledge of the preparation of teachers on state and national assessment data and results that were identified from state teacher certification programs. A review of the literature insinuates that there is a gap in educational research on graduates' experiences and perceptions in field-based teacher-preparation programs. Some argue that it is necessary to modify the amount of field experiences in order to challenge emerging and existing teacher perceptions of the teaching profession (Crawford, 2007; Fletcher \& Luft, 2011; Jones \& Carter, 2007; Lemberger, Hewson \& Park, 1999).

Some field-based teacher-preparation programs differ from traditional teacherpreparation programs in that they require the minimum of one year of field experience and participation in methods courses at their assigned field-based school. Humphrey and Wechsler (2007) argue that in the absence of preparation programs, teachers who are hired to teach develop perceptions that are based on their experiences as students or helpers in a classroom (Darling-Hammond, 2007; Humphrey \& Wechsler, 2007). The hermeneutic theory of Heidegger (1962/1927) and Gadamer (1990/1960) introduces the 
exploration of participants' experiences and develop a deeper understanding of this researcher's personal position, which guides the research process and facilitates how to make sense of the data gathered.

\section{Need for Study}

A review of the literature on field-based programs nationwide indicates that such programs are a growing phenomenon that offers longer authentic field experiences for future teachers. Current research on field-based programs examined whether longer field experiences contribute to the preparation of teachers for the teaching professions (Darling-Hammond, 2005; NCATE, 2007) and whether field-based teachers experience meaningful dialogue with classroom teachers or principals as part of their preparation (Beisser, 2008). A field-based teacher-preparation program is not a new phenomenon in the field of education (Adams et al., 2005; Cristo \& Gimbert, 2002; Zeichner \& Melnick, 1996). A review of educational research demonstrates a critical need to further the relative contributions of field-based teacher-preparation programs in order to identify whether participants are being prepared for the teaching profession.

In Preparing Teachers for a Changing World (2005), Darling-Hammond suggests that teacher educators and researchers have pondered for many years how teachers learn and develop for the teaching profession. Clearly, more research is needed to identify whether these programs prepare teachers adequately for the profession. Seidman (2002) validates the argument that much research is done on schooling in the United States, yet very little is based on studies involving the perspectives of the students and school communities whose individual and collective experiences constitute schooling. 


\section{Interpretivism Literature Review}

Interpretivism is critical because it helps to elucidate how the experiences have prepared the graduates for the teaching profession. It provides the researcher and the participant a foundation from which a method of inquiry can emerge to analyze and reflect on their experiences and identify perceptions of the preparation received. It provides an understanding that what is being researched is an interpretation of what is being studied. While there are many detailed categories of interpretivism, those that brought us to the theory that informed this study are social interpretivism, critical interpretivism, and the newly introduced term, sociocritical interpretivism. Within sociocritical interpretivism, an explanation of language, culture and experience (LCE) in the field-based program provide context for this study.

\section{Interpretivism: A Working Definition}

The term 'interpretivism' is used to imply strategies used in the field of sociology to interpret the implications and actions of actors according to their own subjective frame of reference (Blaikie, 2007; Gerring, 2003; Taylor, 1994). Winch and Taylor define interpretivism as the linguistic interpretation of actors' meanings (Winch 1990/1958; Taylor 1994); here interpretivism is integrated with observation techniques during the interview, which seek to understand the participants' actions and language within the field-based program.

Gerring (2003) alludes that interpretivism may be viewed as a form of 'truthconstruction' that relies on test with external reality. He contends that meaning is derived by analyzing the context of an action or event and identifying how the surrounding set of 
actions, events, and interpretations are connected. In this context interpretivism is an epistemological stance to underscore educational research. Blaikie (2009) presents interpretivism as a theory that originated in the works of hermeneutics and phenomenology, which was essentially concerned with the difference between the subject matters of the natural and the social sciences that requires a scientist to invent concepts and theories for description and explanation (Blaikie, 2009). In addition, Blaikie explains that interpretivism warrants the development of concepts and theories for description and explanation, studying the nature from the 'outside' and making choices on what is relevant to the problem under investigation. By examining the social phenomena from the perspective of interpretivism, social phenomena demands an understanding of society that people have created and reproduced through their on-going activities. However, interpretation is an on-going process of personal experiences, encounters with others, self-encounters, which develop meaning during social activities, and the understanding of what is necessary to make sense of the various activities. To this point, social experience is interpreted prior to the arrival of the participant or researcher (Blaikie, 2007).

Conversely, Williams' (2012) position states that we should use data to generalize during the interpretive process. Williams advocates that, although interpretivists take a broad view, they deny the possibility of oversimplifying. Guba and Lincoln (1994) maintain that it is impossible to oversimplify the interpretive process. Williams advocates that interpretivism must employ a special kind of generalization called 'moderatum', which should limit the use of generalization in interpretivism. Current research on interpretivism illuminates the theories of Weber (1996), Schutz (1899-1959), and Winch 
(1926-1990) as an integration of hermeneutics and positivism, grounded in "German intellectual tradition" and in "British ordinary language" (p. 124). Weber contends that the social world cannot be described without interpreting how individuals use language and symbols to construct what social practices and experience mean to them. Additionally, justification of the social behavior could not be complete unless it adequately describes the role of meanings in human actions.

Blaikie (2007) maintains the founders of interpretivism followed the 'Classical Hermeneutics' theory, which pursued and established an objective science of subjectivity, with the aim to validate the knowledge of meanings that constitutes the social world, an appeal to an imagined whole (Blaikie, 2007; Gerring, 2003). The central tenant of interpretivism helps to derive meaning through social actions, patterns in social life, and the nature of the meaning. Weber (1964), Schultz (1962), and Winch (1958/1990) argue that the interpretivists question the relationships between social actor concepts and meaning, and the concepts and meaning used in identifying social theories. Conversely, Schwandt et al. (1994) argue that interpretivism entails the ability to discover how the nature of the social world brings meaning to individuals through their daily interactions. Meaning interpretivism could be misleading because of the ongoing behavior monitoring, regulation, and justification of behavior.

Holstein's (1993) theory of interpretivism involves approaching and emphasizing the meaning of the nature of people's participation in the social and cultural world. Further, he indicates interpretivism occurs when the researcher and participant articulate and engage in on-going dialogue about the experience. Interpretivism allows the 
researcher to understand the perception about ideas and experiences that are important (Blaikie, 2007; Gerring, 2003; Holstein, 1993). Gerring (2003) also contends that "all" interpretivists agree that their kind of research (a) searches for "inter-subjective" meanings, which are contrasted with "subjective" meaning and "objective" facts; (b) views the importance of attending to the meanings that are attached to the set of actions or events by the participants under study; and (c) describes interpretivism as being "selfconsciously actor-centered" (p. 2). Therefore, it is understood that interpretivists periodically view the textual as the object being studied.

\section{Interpretivism as Related to Education}

Educators are concerned with addressing the needs of students in the classroom.

In order to address these needs, educators perform pre- and post-assessments to determine the strategies that can help students become successful. Interpretivism emerged in the field of education near the beginning of the $20^{\text {th }}$ century to define the difference between the nature of the phenomena investigated by the natural sciences and the nature of those studied by historians, social scientists, and educational researchers.

Interpretivism can be used as a form of pre- and post-assessment to identify how to address the needs of field-based participants in the schools. Interpreting the experience of the graduates from their own perspectives ignites a higher level of learning. This interpretation shows how the preparation of teachers for the teaching profession can be improved; it guides curriculum and communicates how graduates perceive the preparation received from the program. Kvale (1996) recommends that the "research interview is a conversation about the human life world, with the oral discourse 
transformed into texts to be interpreted" (p. 46). Smith et al. describes in-depth interviews as a "purpose to inform." In this context, the FB/TPP is the "human life world," where interpretivism encourages open dialogue to identify how each experience prepares graduates differently (Kvale, 1996). Additionally, the theoretical construct of interpretivism allows participating universities to explore various venues that afford authentic experiences, self-reflection, and self-evaluation.

Interpretivism can assist in the development of educational data and policy and assist in analyzing present procedures and theories in teacher development. Interpretivism can strengthen the basic understanding of issues concerning methods that assist in problem solving in research (Smith, et. al, 2010). Most importantly, the researcher develops and interprets his/her research and methodology skills. Educators are constantly interpreting the behaviors of the students, teachers, parents, and administrators to derive meaning. The teacher and school community also interpret the school experience to gain knowledge. Interpretivism allows the exploration of the experiences to improve the overall experiences in the school and social constructs. From the viewpoint of the researcher, interpretivism allows the interpretation of the learner and school community and allows educators to become familiar with interpretive theory.

\section{Interpretivism as Related to This Research}

Interpretivism is a foundational component of this research study in that the theoretical foundation enables educators to engage in a self-reflective process to analyze their field experiences and perception of the program in which they participated. The researcher engages in dialogue with the participants from a field-based teacher 
preparation program to identify themes that emerge from their perspectives. The researcher and participants engage in dialogue, reflection, and interpretation of their field-based experience while in the program.

In this construct, the research explores the perception of their personal and social world, experiences, and events (Smith, 2010; Schram, 2001; Kvale, 1996). Interpretivism fosters the implementation of phenomenology as a method of inquiry in that it enables phenomenology, gathering, analyzing, and interpreting such data. Phenomenology as a research method furthers the aforementioned tenants of interpretivism because it provides a seamless progression from theory to methodology and presentation of pertinent information as it relates to field-based teacher preparation.

Schwartz-Shea and Yanow (2012) state "interpretivism seeks to understand what a thing is by learning what it does and how particular people use it in a particular context" (p. 151). Interpretivism focuses on context-specific meaning rather than seeking generalized meaning derived from particular contexts. This interpretive field-based flow of inquiry differs from other works that demonstrate interpretivism in the form of text. This flow of inquiry communicates the concept of social and critical interpretivism, especially as it relates to language, culture, and experience. 


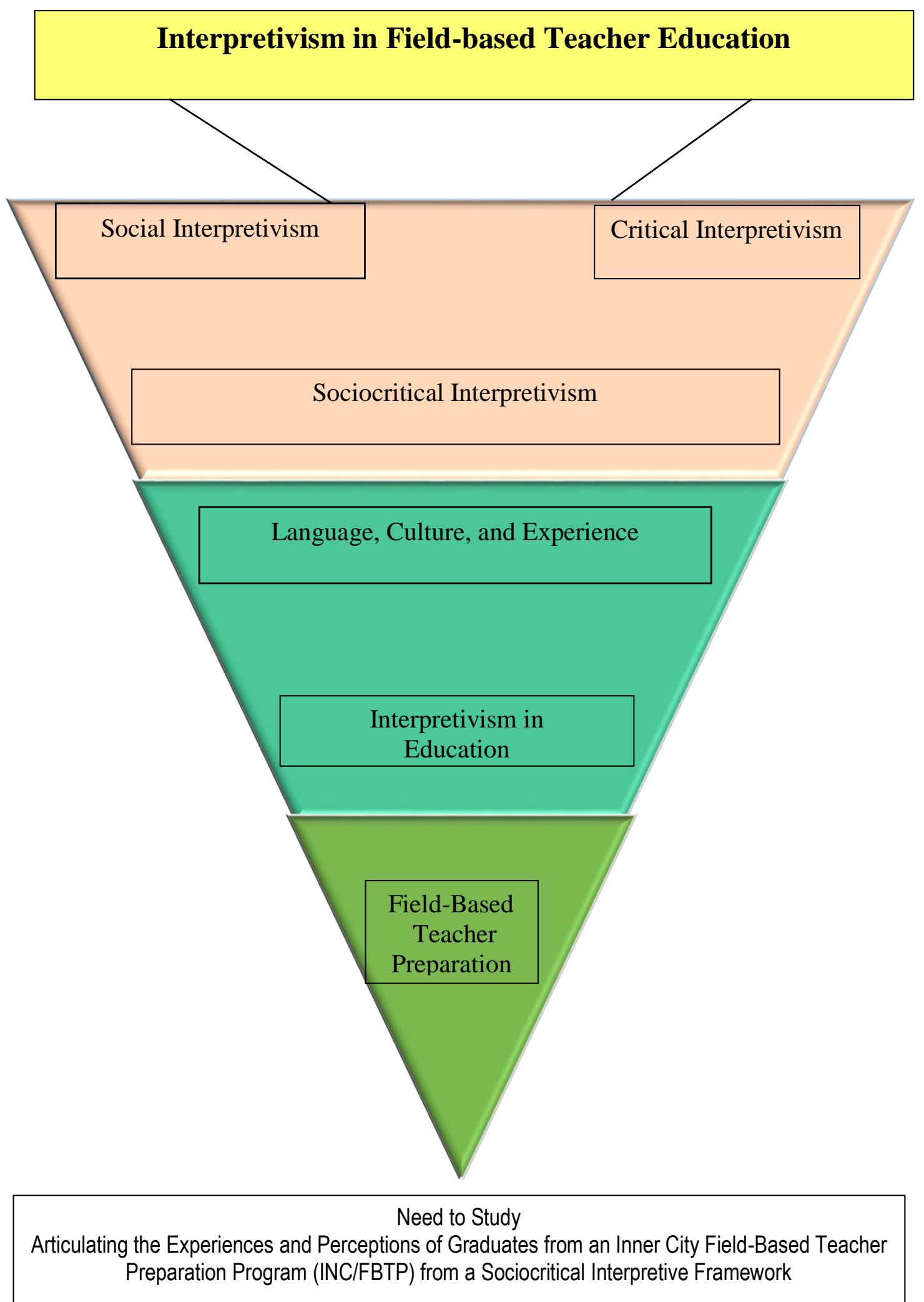

Figure 3. Literature Map and Focus of Inquiry 
Figure 3 presents a conceptual model of interpretivism in field-based teacher education. The figure demonstrates the flow of inquiry from left to right that formulates the model social critical interpretivism. Two major conceptual distinctions social and critical interpretivism, and language, culture and experience are further outlined in this chapter.

\section{Social Interpretivism}

Social interpretivism enables the understanding of how the social world influences experiences, which, in turn, is collaborative between or among people. Interpretation occurs in our daily lives or work experience, within the social context. Behaviors and actions all occur within structure of position, hence, in this context, experiences are then viewed as socially mediated. This perspective provides a comprehensive understanding of multiple social and organizational modalities (Rolands, 2005; McLaren, 2007; Schwandt, 1997). The concept of social interpretivism is adopted and applied from the social science fields and the interpretivist paradigm, and it is then applied to the field-based teacher preparation programs. Social Interpretivism is an epistemological assumption where there are multiple implications that are derived from social interaction and have different meanings relative to others (Erickson, 1986; McLaren, 2007; Rolands, 2005; Werstch, 1991). Meaning and knowledge are socially constructed within the context of field-based teacher preparation programs, specifically as related to field-based participants. Klien and Myers (1999) argue the assumption for interpretive research in that knowledge is gained or at least filtered through social constructions such as language, consciousness, and shared meanings (Klein \& Meyers, 
1999; McLaren, 2007; Rolands, 2005). Blaikei (2007) agrees with many theorists regarding how the social environment is constructed through daily on-going interaction and plays a vital role in the understanding of the social world. In essence, social interpretivism is an on-going process where people begin to develop meaning for interactions and assist in the ability to visualize the work experience (Blaikei, 2007; Bradley \& Levinson, 2007).

Research shows that there are number of emerging trends explaining the social interactions in the field of teacher preparation, but more is needed in the area of fieldbased teacher preparation programs. According to Schoeman and Mabunda, (2012), current social theory focuses on learning to teach and the socialization process, which occurs from the first day of exposure to the classroom (Burn, Hagger, Mutton, \& Everton, 2000; Robinson, 1999). As such, field-based teacher preparation programs are heavily influenced by the social interactions between the field-based participants and the school community, learning experiences, and supervisors' support (Schwandt, Denzin, Norman, Lincoln, 2012; Westbrooks \& Alea, 2007; Flores, 2007; Dean, 1996).

Social interpretivism also involves understanding the language and cultural practices as they relate to field-based teacher preparation programs and the social interactions in the field. Additionally, it explores how the social relations in the fieldbased program foster competitiveness, cultural behavior, and equality within the program and among the program participants. It explains how and why the program functions off campus in a collaborative worksite. Curriculum must be viewed from the perspective of "theory of interest" and "theory of experience" (Giroux, 2011). Theory of experience 
proposes that field-based experience should be socially organized to reflect the social constructs of language, culture, and power to develop participants in the field-based teacher preparation program (Giroux1990; McLaren, 2006).

\section{Critical Interpretivism}

Joan Wink (2005) defines critical pedagogy as the ability to have a clear understanding of what is being said, pondered, critiqued, or analyzed as the learning experience transforms in the classroom (Giroux, 2011; Wink, 2005; McLaren, 2007; Hinchey, 1998; Freire, 1993). Critical interpretivism is closely linked to forms of control, issues of justice and equality in relation to the process of collecting, and interpreting data (Giroux, 2011; Wink, 2005; Hinchey, 1998; Freire, 2005). Critical interpretivism enables researchers to use, adapt, and apply concepts to deepen their understanding of social experiences and field experience to develop as agents of change. For this purpose, critical interpretivism is presented to comprehend research in teacher education, especially as it relates to teacher preparation programs. Critical interpretivism supports the understanding of how decisions are made in the field-based program and how the program can serve as an agent of change toward teacher preparation. It enlightens the perceptions and perspectives of graduates who participate in the program and their respective preparation. Additionally, critical interpretivism facilitates a process in which the researcher learns to interpret the experiences in the field as told by others relative to field-based teacher preparation programs. Many researchers place great emphasis on questioning, as did Freire (1993), Hinchey (1998), Williams (2012), and Wink (2005), in their discussion on critical pedagogy. According to Hinchey (2010), critical theory uses specific questions 
to guide. "Who has power? How did they get it? How did they keep it? What are they doing with it? How do their actions affect the less powerful (19)?" In addition, how might things be otherwise to guide our understanding of issues of our world (Freire, 1993; Giroux, 2011; Hinchey, 1998; Williams, 2012; Wink, 2005)?

Based on the aforementioned questions, critical interpretivism, as related to this study, requires a response to the following questions: What type of power structure is prevalent in the field-based teacher preparation program? What specific field experiences support the participants' sense of self and place? How do graduates think field-based programs function to promote change? Critical interpretivism enables interpretation of data and knowledge of the graduate's field experience by asking critical questions. Quintessentially, it explores how program decisions are made and how the choices affect groups differently, who makes the decisions that affect everyone in the group, and who empowers these individuals? In addition, the following questions were adapted from Williams' (2012) Critical Issues in Literacy Pedagogy: Notes from the Trenches to assist in addressing the following issues of concern in field-based teacher preparation. They are:

1. What are the benefits of field-based teacher preparation as it relates to teacher preparation?

2. Who benefits from the program and why does field-based programs privilege one group over another or all?

3. Does a field-based program serve to maintain inequalities of power? Critical interpretivism involves interpreting the experiences in the field-based 
program as they relate to learning, classroom pedagogy, language, and culture.

Additionally, critical interpretivism involves interpreting how participants interact in the program, in the social world, with professors, teacher mentors, and parents. Williams (2011) states students learn effectively when there is evidence that the learning environments are conductive to learning, and promote equality. Critical interpretivism transcends traditional methods of interpreting critical thinking and questioning of the social world, in this case, the field-based teacher preparation program. It is very important to understand how field-based teacher preparation programs influence classroom pedagogy and teacher preparation.

Another important component of critical interpretivism includes interpreting the experiences of graduates in concrete and applicable terms. The researcher gains insight into the experiences as it relates to the issues of power, equity, language, and culture. Critical interpretivism enables the researcher to question those in power and assist program evaluators in the development of future programs. As a critical interpretivist for this study, the researcher asked questions that promoted the participants' abilities to reflect upon their field experience and how those experiences shaped their professional classroom pedagogy. As critical issues and complexities in field-based programs are researched, more than one experience in the field-based teacher preparation program deserves investigation. Many of these experiences can be linked to class, race, and power issues. As the experiences of the graduates from INC/FBTP were explored, research should be extended to explore how an individual's beliefs and language contribute to the 
culture and experience of the program. According to McLaren (2007) "knowledge is socially constructed and heavily dependent on culture, context, and experience" (p. 197).

\section{Social Critical Interpretivism}

Levinson (2012) defines social critical theory as an encounter that seeks to clarify the rationale for organizational power and discrimination in society, necessary to foster individual freedom and impartiality. According to Levinson (2007), social critical theories grew from individual rights and civil liberties to democracy and capitalism. Many theorists have combined a range of strategies to analyze educational discourse and its relationship to social behavior. Similarly, contrasting theories, such as social linguistic theory, critical race theory, social learning theory and critical interpretivism, have been used to discuss educational phenomena.

More recently, social and critical theories have been integrated as a means of analyzing field-based participants as related to social status in field-based teacher preparation programs. It provides a framework for educational researchers to investigate possible concerns of inequality in the field-based teacher preparation program and seeks to explain or justify how field-based teacher preparation programs reinforce or preserve diverse levels of power in field-based teacher preparation programs. A major goal of social critical interpretivism is to develop a clear dissimilarity between the inaccuracies, socially plagiaristic notions that are developed among disregarded or underrepresented groups in field-based teacher preparation programs. 


\section{Sociocritical Interpretivism}

Sociocritical interpretivism (SOCI) was employed to explore the intricate nuances needed to understand the experiences of participants in the field-based teacher preparation program. Additionally, it allowed us to dive deeply into the field-based teacher preparation program to understand how and why the program functions as it does, and the position of the field-based teacher preparation programs within the field of education. For many years, schools served as the place for social order and discourse. Conversely, some theorists argue that schools have the tendency to work against fostering social empowerment. However, being part of a social world includes our daily interactions and social behavior. The discourse of the field-based teacher preparation program can assist in identifying changes and transformation in the field of education and new challenges for theory and pedagogy (Best \& Kellen, 2001). Norris (2012) advises that mediated behavior is a permanent behavior that is always a component of the experience: engaging in dialogue, expressions, practices, sharing, and experiences, etc. According to Norris (2009), a mediated discourse is an encounter taken by a person in the social world with thoughtful measures or resources (Norris \& Jones, 2005; Scallon, 1998).

Heracleous (2000) discusses social critical interpretivism as a consequential understanding of an actor's frame of reference and the key concept that separates the social from the natural sciences (Weber, 1949). Further, he expands Erickson's (1996) social critical interpretive theory as introduced as social interactionism. He contends that there are multiple realities that derive from social interaction and contain various 
meaning for others. Heracleous adds that meaning and knowledge are socially constructed within the realm of context, during specific times, with specific people (Heracleous, 2012). Social critical theories seek to analyze the perceptions of the experiences as they are told during the interview when the participants are engaged with the intention of researching the various topics or the settings (Schwarts-Shea \& Yanow, 2012). Additionally, Rowlands (2005) argues that a person can assume that "Individuals create and develop their own individual idealism as they engage with the world around them" (p. 93).

Sociocritical interpretivism allows the researcher to gain a clear and precise focus of discourse and the central role equity and power play in understanding the many issues involved in field-based teacher preparation programs. Social critical interpretivism examines the existing ideologies and practices of the field-based teacher preparation program and the need for educational and social reform to prepare field-based participants for the teaching profession. Social critical interpretivism further explores the social context of engagement, language, and culture as it relates to such programs. Furthermore, sociocritical interpretivism provides the opportunity to explore the role of criticism to determine if field-based teacher preparation programs provide quality education. Leonardo (2004) advocates that quality is proportional to the depth of analysis that field-based participants acquire during their participation in the field-based teacher preparation program. SCI explores the program discourse analysis and the participants' ability to analyze the field-based teacher preparation experiences that result in empowerment. 
In the field of education, it is important to understand how sociocritical interpretivism is applied to the teaching profession and field-based teacher preparation programs. Sociocritical interpretivism (SCI) enables us to understand the role of power and how field-based participants transform their professional practices. Levinson (2007) advises that sociocritical interpretivism command that the researcher make clear that which is normally understood as assumption. SCI supports the field-based participants' and researcher's understanding of their assumptions by critically examining the significance of field-based teacher preparation programs in the field of teacher education. Sociocritical interpretivism is important in the field of education because it demonstrates the relationship of LCE and provides the researcher with a foundation for engagement with the participants in the teaching profession. From the perspective of education, sociocritical interpretivism helps us gain a clear understanding of how the field-based teacher preparation program functions, the educational process, participant engagement, placement of participants and the roles of professors, mentor teachers, and supervising instructors in collaborative schools.

Sociocritical interpretivism enables the researcher to analyze the social world and the role of schooling and policies while maintaining and challenging social practices in the field-based teacher preparation program. It opens doors for many educational researchers to engage in and advocate for reform in the field of teacher preparation in that it is informed by personal and social interests that determine educational policy and practices (Levinson, 2007; Aggers, 2006; Kyung-Man Kim, 2005). As researchers ponder critical issues and complexities involved in field-based teacher preparation programs, 
there are many experiences in the field-based teacher preparation program that deserve investigation. The program experiences can be linked to class, race and power issues. As the experiences of graduates from an INC/FBTP are explored, research should be expanded to explore how an individual's language contributes to the culture and experience of the program. According to McLaren (2007), "knowledge is socially constructed and heavily dependent on culture, context, and experience" (p.197). Sociocritical interpretivism lends itself to language, culture and experience constructs in education, which influences the meaning of field-based teacher preparation programs.

\section{Language, Culture, and Experience}

Language, culture, and experience (LCE) play a significant role in field-based teacher preparation programs. It is worth considering what these concepts have to do with teachers' perceptions of the preparation they received (Lytra, 2011; Blackledge \& Creese, 2010; Conteh, Martin, Helavaara, \& Robertson, 2008; Lytra \& Barae, 2009). One way these can be explored is how they either positively or negatively impact the perceptions of teachers involved in field experiences. According to Geertz (1979), language and culture significantly influence the educational discourse in relating to social application (p. 218).

The use of language, culture, and experience (LCE) changes over time and varies according to the situation. For the purpose of this study, language is defined as the ongoing verbal and non-verbal cues (behaviors) that constantly change through interaction and communication with other participants in the field-based program. Language in this context of culture is an identifier and shapes culture and influences ways in which people 
communicate (Lytra, 2011, Sigh \& Han 2010; Blackledge \& Creese, 2010; Hayes, 2009;

Contech, Martin, Helvaara, \& Robertson 2008; Lytra \& Barae, 2009; Raminsky, 2003;

Finnan, 2000; Bruner, 1996; Geertz, 1979).

Shohamy (2007) defines language as ongoing and personal, which encompasses the nuances of communication. She proposes that it is a means of viewing, comprehending, and verbalizing in the social world. Kramsch (1994) also argues that language is a "societal behavior" that people perform daily, creating and interpreting meaning. Raminsky (2003) maintains the differences in the social roles influence how language is used. He notes the differences in how home and school contexts represent different cultures, subcultures, or both, and may influence language acquisition in different ways. Interpretivists would analyze non-verbal cues (e.g., facial expressions and body language) and contextual cues (e.g., shared field experiences) to explain the different experiences from the participants. With this being said, language, culture, and experiences (LCE) are analyzed in the context of interpretivism to understand how fieldbased teacher participants articulate and communicate their experiences. The culture of the school community and the field-based teacher preparation all reflect the value that develops during participation in the program. Utley et al. (2001) define culture as the following:

Culture is more than just one characteristic, such as race or ethnicity; culture reflects the unique blending of characteristics among individuals within groups and may include variables such as socioeconomic status, life experiences, gender, language, education, sexual orientation, psychological state, and political viewpoints. (p. 8) 
Current research alludes that language and culture (LC) share a common conceptual core and a set of related assumptions regarding experience. LC can be used to interpret how experiences are analyzed. In critical interpretivism, the researcher and participants develop a level of trust, at which point interpretation begins.

Acculturation is the main challenge for participants in a field experience. As a result, this new field experience influences participants' perceptions of their own identities and professional teaching career (Singh \& Han, 2010; Hayes, 2009). Our perceptions are altered by conversations and individual interactions in the field. Every field-based experience is the result of what is seen and heard, and otherwise experienced daily. The social interpretation in the program influences individual choices during the interpretative process (Finnan, 2000). Field-based participants are learning the discourse and nuances of field-based programs; language lends itself to the social critical interpretive theory, which entails social practices and develops meaning of the fieldbased participants' experiences. Zaid (2011) suggests that the social-cultural environment serves as a critical element for language, cultural identity, and learning. Field-based participants possess their own cultural thought process and expectations of the fieldbased program. As the participants actively engage in the field-based program, they begin to identify with the role of their new culture as teachers (Brislin, 1993; Geertz, 1973; Goodenough, 1971; Hinde, 2002; Murrell, 2007; Nieto, 2000; Rice, 2010).

In addition, Zaid (2011) put forward that a cultural environment is a crucial element for cultural learning and the sociocultural environment, because it provides the most essential component for language and cultural identity experiences (Abel, 2002; 
Hung, 2008; Varghese et al., 2005; Zaid, 2011). Everyone participates in a form of learning a culture via communication, working, social, school and home, which is ongoing, enhanced, and shared in our social world. Researchers view culture as a constant and unchanging unit that can be accumulated, classified, and observed (Brooks, 1975; Nostrand, 1974). Interpretivists analyze the role of the participant in the development of culture and the interactions of language and culture in the understanding of meaning. Paige et al. (2012) call to mind that people emulate a number of observable behaviors and varying levels of attention to the ongoing importance and meaning constructed through daily language and cultural experiences (Siaya, Jorstad, Siaya, Klein, \& Colby, 2012).

Language is used in a sociocultural environment to identify the socio-political and economic tenets that analyze how language, culture, and experience develop the individuality of learners in the social and cultural perspective (Barac, 2008; Issa, 2008; Lytra \& Lytra, 2011). Sociocultural perspectives entail a strong development of consideration and thought provided by a person's culture acting with consideration and thought in particular sociocultural moments (Wertsch, Bakhtin, \& Vygotsky, 1991).

\section{Language, Culture, and Experience (LCE) in the Community}

Everyone interprets environments that are rich in language and culture differently. It provides a growing abundance of cultural experiences that aid in professional development in the teaching profession. Researchers and educators, such as Goddard and Woolfolk (2000/2004) and Tschannen-Moran, Woolfolk, and Hoy (1998), evokes if the issues of culture, language, and experience are addressed in field-based programs, 
participants can develop their cultural awareness and self-worth. In investigating fieldbased teacher preparation programs, sociocritical dynamics explore participants' interpretations of instruction and communications among peers, with mentor teachers, and professors. The following questions deserve exploring to comprehend the role of language and culture (LC) in such programs: How does the field-based teacher preparation program result in the development of the teacher's identity and learning? In addition, how is language and culture in field-based teacher preparation programs' education setting/context defined? Immersion in a field-based community would allow the participants to make connections in the teaching profession.

Additionally, language and culture (LC) in the school community involves school-wide mandates, which guide daily instruction, the community where teachers work, field-based teacher preparation program, and the collaborating schools. The LC in the school community indicates the daily practices and perceptions of information and how the information is interpreted. LC in the school community could affect the manner in which field-based participants perceive the teaching profession, the delivery of instruction, as well as how they understand the school community as a whole. The culture of the field-based program encompasses the body of knowledge about the characteristics of the program. LC is critical because it facilitates dialogue with the entire community.

\section{Conclusion}

Research was completed on student teaching, field experience, and teacher preparation. However, very little research addresses the orally shared experiences and 
perceptions of graduates who have participated in these programs and, in particular, fieldbased teacher preparation programs.

For many years, teacher-preparation program success factors were dependent on surveys, teacher success on state assessments, and student teaching performance. A new means is needed to assess the success of what preservice teachers are learning and how the field experience has prepared them for the teaching profession. Many researchers posit that field experiences provide teachers with an opportunity to connect theory to practice more effectively (Beeth \& Adanan, 2006; Tigchelaar \& Korthagen, 2004). It is important to understand the experiences of field-based participants in order to assess whether field-based teacher-preparation programs "better prepare" future teachers by linking theory to practice. Field-based programs deserve exploration to analyze the perceptions of participants regarding mentor teachers, coaching, and the goals of the program. Policymakers, state boards of education, colleges, and reformers might find this information beneficial to improve teacher preparation field experiences and to use the experiences and perception as a tool to guide policy and to reform initiatives. Interpretivism lends itself to phenomenology, a method to collect data on the experiences of the participants and enables the researcher to analyze the data, so their experiences are explored and analyzed.

This literature review identified a lack of research in perceptions of participants in teacher preparation programs and, in particular, field-based teacher preparation programs. Through this study, the perceptions of field-based participants are examined to answer the overarching research questions. 


\section{CHAPTER III}

\section{METHODOLOGY}

The primary focus of this chapter is to provide insight on the participants of the study, discuss the research on the experiences and perceptions of participants from an inner city field-based teacher preparation program (INC/FBTP), and explain how the research was conducted. It was critical to analyze important issues that contributed to these emerging and evolving perceptions. Interpretivism as a theoretical framework, in conjunction with phenomenology as a method of inquiry, allowed the researcher to consciously comprehend, interpret, and make effective judgments about critical components of the field-based teacher preparation program as it relates to the teaching profession.

Although there are numerous recommendations for educational reform on teacher preparation, little research has been conducted on the experiences and perceptions of participants in field-based teacher preparation programs to ascertain if they are fully prepared for the teaching profession. The implications for teachers, practitioners, and stakeholders in the wider education community is relevant and provides clear and concise issues and concerns from participants in the field-based program regarding their perceptions of preparedness, knowledge, skills, and attitudes, and relationships and experiences with professors and mentor teachers that are critical for success. The methodology provided a basis for the researcher to document specific experiences, as 
perceived by the participants. It was necessary to understand the meanings that field experiences had for graduates and how they envisioned the teaching profession. An interpretative phenomenological qualitative research construct was used for this study. This epistemological framework hinged on the participants' personal experiences, ways of knowing, and responses to the field-based teacher preparation program.

\section{Research Design}

Phenomenology was chosen as a method of data collection because it unfolds the meaning of the experiences and perceptions of graduates from the program. This method assisted the researcher in identifying the individual experiences about a phenomenon as portrayed by the participants (Creswell, 2009; Gall et al., 2007). It was adopted as a method to collect data on the experiences of the participants, analyze the data, and enable the researcher to explore the participants' experiences. Recognizing that this research study answers questions related to the experiences of graduates who initially participated in a traditional teacher preparation program and later registered for the field-based teacher preparation program provided an understanding regarding the level of preparedness participants believed they had for the teaching profession.

Critical to this research was providing an interpretation of the participants' experiences in order to gain in-depth knowledge of their experiences and perceptions in the field-based teacher preparation program. Seidman (1996) proposes that, in phenomenological qualitative research, the interviewer makes deductions by conducting three in-depth interviews. Coding was generated to create themes from the interview data and appear as major findings in the study. They were used as headings and demonstrated 
numerous participant perspectives that were supported by concrete data. The researcher then entered the data into a database according to analysis headings (Creswell, 2009; Lester, 2007). The researcher transcribed the interview by transposing the data from the oral interview to words on a typed document. The interpretations were coded from themes identified from the interview, not from the researcher's interpretations alone. Most importantly, this interpretation came from the perspective of the participants.

\section{Tenets of Phenomenology}

For the purpose of this study, the following tenets of phenomenology were critical for analyzing, explaining, and presenting crucial information that was utilized by the researcher.

\section{Listening}

The researcher engaged in three levels of listening: active, inner voice, and assessment. Active listening to the experiences of the participants required concentration to assure that the researcher clearly comprehended what was articulated and listened for details. Listening for the inner voice allowed the participants to describe the experience and feel comfortable in sharing the experience. Last, listening to assess the interview focused on the process and content, made the most use of the time provided, and allowed the interviewer to be attentive to nonverbal and verbal cues (Schwartz-Shea \& Yanow, 2012).

\section{Conversation}

Critical in phenomenology are the conversations between the researcher and participant. Conversing explored the construction and process of the conversation where 
the researcher's comprehension was developed and maintained. The researcher focused on what was being said, as well as inflection in the participants' voices during the conversation. Understanding for meaning and the sequence of the conversation was also important at this stage (Kvale, 1996; Schwartz-Shea \& Yanow, 2012).

\section{Validating the Experience}

A collaborative co-construction and interpretation of the experiences between the researcher and the participant validated the experience during the interviews. The researcher ascertained internal consistency, clarified ambiguity, and arrived at shared understanding about the meaning of the experience. Validation became critical as the researcher focused on understanding the participants' experiences, the flow in the dialogue, and articulation of verbal and understanding of non-verbal cues (Giorgi, 2009; Kvale, 1996; Smith, Flowers, \& Larkin, 2009).

\section{Describing the Experience}

The researcher must allow for the complete description of the experience as defined by the use of language to articulate the intentional focus of experience (Giorgi, 2009). The experience is made authentic by exploring the nuances related to the

phenomena. The researcher must read the interview in its entirety before the description begins. According to Creswell (2012), it is critical that the experience is articulated as clear and concise as possible without any additions or omissions so the necessary emerging themes will evolve. 


\section{Interpreting and Analyzing the Experience}

The researcher sought to derive meaning of the participants' experiences by actively listening, interpreting the meaning of what was said and how it was said. During the interpretive stage, the researcher was observant and capable of interpreting vocalization, facial, and nonverbal cues. Additionally, the researcher tried to clarify ambiguity from the interviews as presented, which was necessary, to understand meaning, and make connections to the experience (Giorgi, 2009; Kvale, 1996; Smith, Flowers and Larkin, 2009).

\section{Disclosure of Data}

The researcher worked from the findings and applied analytical procedures to develop concrete interpretations. The researcher was cognizant of potential biases and the level of sensitivity about the participants' experiences. Data was reproduced in a manner that restricted the identity of the participants, mentors, professors, and schools. Most importantly, data, was readable, coherent and concise (Kvale, 2007).

\section{Researcher Bias}

Apparent in qualitative research, biases affect the reliability and validity of the findings and the data. Biases have the tendency to skew the perception of the researcher and the data that is being transcribed. Specifically, age, race, gender, facial expressions, body language, tone, and gestures may introduce a level of bias to the participant. It is critical that the researcher excuse personal experiences and maintain an open mind to allow the phenomena to emerge in order to understand the experiences of the participants in the study (Giorgi, 1997; Seidman, 2006). Personal experience as an elementary 
student in an inner city public school and former field-based participant played an important role in potential biases for this study. Additionally, the manner in which the researcher asked the interview questions could lend itself to bias. The researcher used Kvale and Seidman's interview guide to reduce biases by rephrasing or removing specific questions.

As the researcher and former field-based graduate, there was a possibility that the field experience could affect personal perceptions and interpretation of the data. Thus, the researcher was aware of personal perceptions and interpretations and tried not to allow these perceptions to intrude on the interview and data analysis processes. Personal knowledge about the field-based program allowed for a deeper understanding of the issues and concerns brought by the participants. Kvale (2007) argues that preconception can hinder the analysis process. However, when conducting this research, the researcher was not a participant. The researcher implemented a number of strategies to insure that personal biases did not skew the research process, data analysis, or the reporting of the results. These strategies included, but were not limited to, remaining neutral, describing experiences in full detail, maintaining an open mind during the interview, member checking to ensure interpretation is true to the participants' original ideas, and data analysis and reporting processes to prevent the biases from skewing the interpretation.

\section{Field-Based Participants}

Participants for the study were selected using purposeful sampling. Purposeful sampling is the ability to identify or select a purposeful group of individuals that will assist in understanding the participants' experiences in the field-based program (Creswell, 
2009; Seidman, 2006; Mertens, 2005; Patton, 2002). A purposeful sampling was conducted for two main reasons: (a) the researcher was able to analyze the perceptions of participants from the INC/FBTP, and (b) the researcher had access to firsthand information from program participants to assist in understanding the phenomena. Data regarding the perception of program preparation were collected to determine how the field-based program functioned and to analyze how the program could be improved to enhance future teacher candidates' knowledge and skills to teach in present-day classrooms.

The sample consisted of four graduates from an INC/FBTP. The inclusion criterion required that all participants met the following criteria: (a) participated in the INC/FBTP, (b) successfully completed the program, and (c) were currently employed as an educator in the field. Participants' contact information was accessed via the university alumni directory, which is publicly available. Participants were solicited via general email or post office and were selected based upon their willingness and availability to participate. Further, participants were notified by email and U.S. postal services to explain the nature of the study and to request their participation. There was no form of compensation for participation in the study, and demographics were not identified nor recorded to protect the anonymity of the participants.

The researcher asked the participants if they were willing to participate in the study. Once the participants consented, the researcher contacted the graduates and explained the study in detail. A meeting time was scheduled at a mutually agreed upon location and time with all of the graduates who agreed to partake in the interview and met 
the inclusion criteria. The researcher mailed a consent letter and contract for the participants to complete and sign prior to the first interview (see Appendixes C \& D). The researcher obtained verbal and written consent to record each participant's interview. Each of the graduates' three interviews were face-to-face interviews, recorded, transcribed and coded to ensure confidentiality, appropriate reporting and data analysis.

\section{University Background}

This research focused on a field-based program at a university located in a middle-class working community in a large urban area. Enrollment at this inner city university consists of a diverse population of predominantly African American and Hispanic students. The community is comprised of a number of highly educated residents, with a large majority of the adults having obtained one or more college degrees. Community members are very active in the educational efforts of the university and its commitment to education. This university is one of approximately 11 higher education institutions located in the large city. Programs offered include a Division of Continuing Education and Non-Traditional Programs, extension sites, extension courses, distance learning, and not-for-credit programs. Like a number of universities in the state, student enrollment declined following the commencement of the field-based program.

\section{Participants' Background}

Although 16 participants responded, only 4 were available for the interview and provided dates for their interviews. The pseudonyms provided for the four participants were Sharon, Lucretia, Yolanda, and LaTonya. The participants for this study were all African American, non-traditional students whose age range was between 25 and 60 . 
They were enrolled in the field-based program at various times between 1994-2013 during the methods section of their academic program, prior to student teaching. Two of the participants were undergraduates and two were graduates pursuing educational degrees and K-12 licensure. The participants were certified teachers while in the program, nor did they have any professional teacher training prior to the field-based experience. However, all of the participants worked in the corporate world prior to pursuing their degree in education, served in leadership and or training positions, and gained experience working with children, teens, and young adults in youth groups and community organizations. 
Table 2

\section{Field-Based Participants' Characteristics}

Note. Field-based participants' characteristics consisted of ethnicity, age range upon admittance to the program, employment and teaching in other careers, the number of years teaching after the completion of the program, the year of graduation from the program and grade level of certification at the completion of the program.

\begin{tabular}{|c|c|c|c|c|c|c|}
\hline Participant & Ethnicity & $\begin{array}{l}\text { Age } \\
\text { range }\end{array}$ & $\begin{array}{l}\text { Teaching } \\
\text { experience } \\
\text { in other } \\
\text { careers }\end{array}$ & $\begin{array}{c}\text { Years } \\
\text { of teaching } \\
\text { experience } \\
\text { after } \\
\text { certification }\end{array}$ & $\begin{array}{l}\text { Year } \\
\text { graduated } \\
\text { from FB } \\
\text { program }\end{array}$ & $\begin{array}{c}\text { Grade level } \\
\text { certified and } \\
\text { employed }\end{array}$ \\
\hline Sharon & AA & $45-55$ & Yes & 17 & 1997 & $\begin{array}{l}\text { Middle } \\
\text { High School }\end{array}$ \\
\hline Lucretia & AA & $25-35$ & Yes & 4 & 2010 & $\begin{array}{l}\text { Middle } \\
\text { School } \\
\text { Higher Ed }\end{array}$ \\
\hline Yolanda & $\mathrm{AA}$ & $35-45$ & Yes & 10 & 2004 & High School \\
\hline LaTonya & AA & $45-55$ & No & 2 & 2012 & $\begin{array}{l}\text { Middle } \\
\text { School } \\
\text { Higher Ed }\end{array}$ \\
\hline
\end{tabular}

\section{Data Collection Procedures}

Semi-structured interviews were conducted based upon an interview guide that was developed by the researcher to address field-based experiences of the graduates in the field through interviews, discussions, and participant observations. This form of interviewing combined life history interviews and focused in-depth interviewing informed by assumptions that were drawn from phenomenology (Kvale, 2007; Seidman, 2007). 
The researcher observed verbal and nonverbal cues of the graduates during the interview. This form of observation helped to connect with the graduates, interpret what they were saying, and built relationships with the participants. Many times, when engaging in dialogue, body language conveys something different from what is said. The nonverbal cues being observed hints at the inner or true feelings during that time. Additionally, observing the nonverbal cues assists in validating the experience and indicates how well someone is listening. When the nonverbal signals match the words that are articulated, they increase trust, clarity, and comfort in dialogue engagement. When the nonverbal cues fail to match, they generate tension, mistrust, and confusion (Patton, 2002).

The interview protocol was designed using the research questions as a framework, to ask questions, record the interview, and gather data. Research questions that guided this study are included in Appendix B. The interview protocol consisted of a set of three separate sets of interview questions. The first set of questions was developed to establish the context of the participants' experiences or to get to know the participant and to determine how each participant became involved in the field-based teacher preparation program. The participants reconstructed their experience with the professors, the mentor teachers, and in the school in the field-based program. The second set of questions caused participants to focus on specific details of the field-experience. Participants reconstructed their experience in the day-to-day operations of the program, and it allowed the participants to construct the details of their experiences within the sequence it occurred. The third set of interview questions was created after the analysis of the first and second 
interviews. These questions encouraged the participants to reflect back on the meaning of the field-experience.

\section{Interviewing}

The interviewer gathered data in the form of tape-recorded face-to-face interviews, hand written notes, and observations during the interview. For the purpose of this research, Kvale's (1996) seven stages of interview investigation and Seidman's (2006) three-stage interview process were used. Implementing both Kvale (2007) and Seidman's (2006) interviewing processes allowed the researcher to record the participants' explanation of their field-based experiences and focus on in-depth interviewing techniques to help interpret and inform the meaning of the participants' experiences (Seidman, 2006). This process provided a template for the researcher to assist in the overall design and implementation of the interview process and protocol. The two processes complemented each other during the data planning, data collection, and analysis stages. The researcher used the two processes to look beyond the surface to develop questions that allowed the participants to be comfortable enough to discuss and reflect on their overall experiences. Additionally, the process helped the researcher relate to, discover, and connect with the participants and the data being analyzed.

\section{Kvale's Seven Stages of Interviewing}

Kvale's (2007) seven stages of interview consist of thematizing, designing, interviewing, transcribing, analyzing, verifying, and reporting. The stages are displayed in Figure 4 to assist in understanding and validating the participants' experiences. 
1. Thematizing. Prior to the start of the interview, designing the purpose of the investigation and describing the concept of the topic being investigated.

2. Design the study. To gain knowledge about the topic, remembering the moral implication and obtaining an intended knowledge.

3. Interview guide. Interview based on the interview guide, with a reflective approach to the knowledge sought.

4. Transcribe. Transcribe the interview material for analysis from oral speech to written text.

5. Analyze the interview material. Decide which method of analysis is most appropriate for the interviews based upon the purpose and topic of investigation.

6. Verify. Determine the generality, reliability, and validity of the findings.

7. Report and articulate the data. Develop a qualitative report that illustrates the methods and concrete findings.

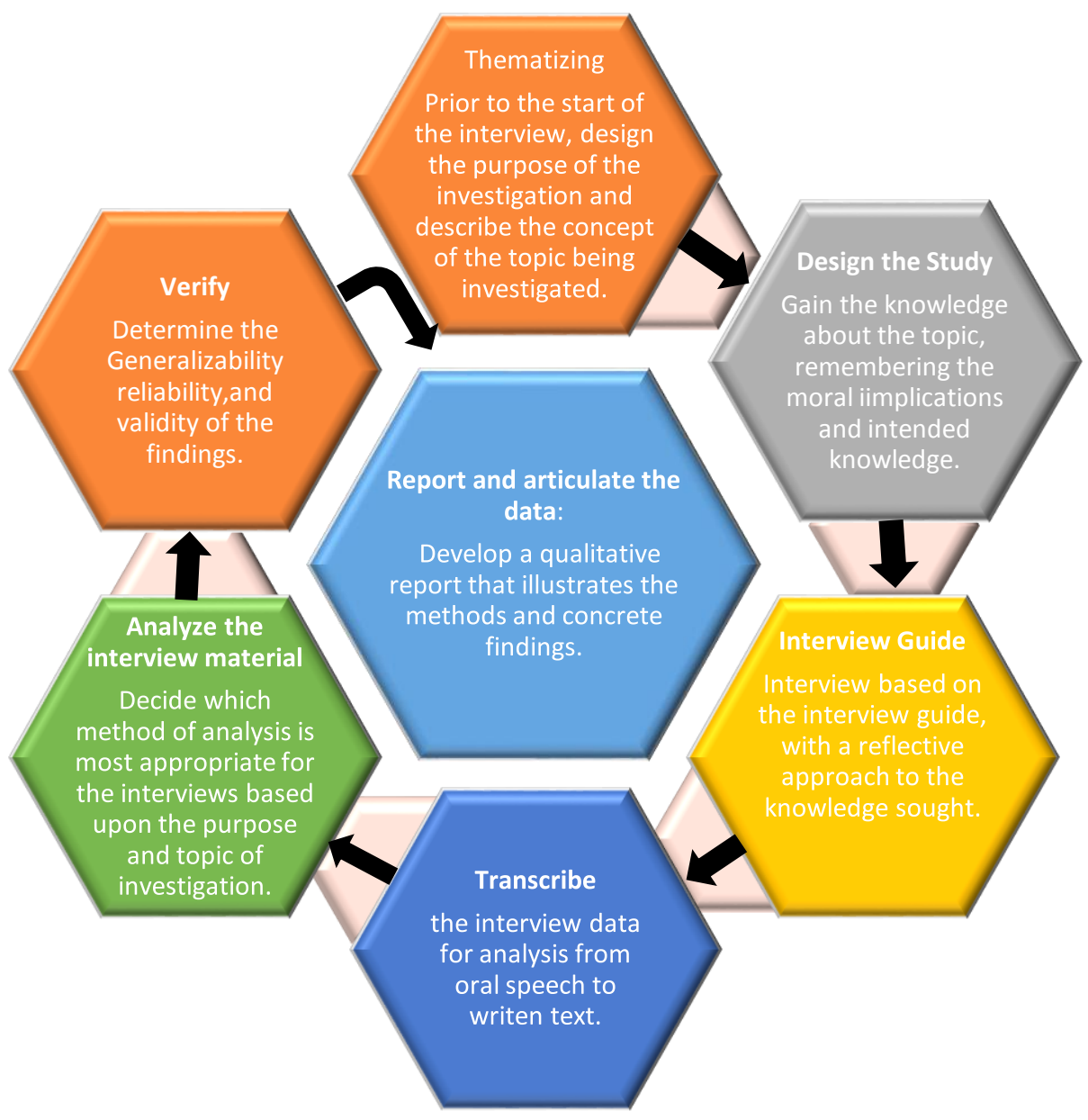

Figure 4. Kvale's Seven Stage Interview Process Used in the Data Collection Process 


\section{Seidman's Three-Stage Interview Process}

Seidman's (2006) semi-structured qualitative in-depth interviewing method consists of a series of three separate interviews that were implemented to explore the experiences and perceptions of graduates from field-based teacher preparation programs. In-depth interviewing is necessary for the researcher to derive meaning of the participants' experiences. Schuman (1982) suggests that the three interviews allow both researcher and participant to reflect on the experience, place it in proper perspective, and (co)construct meaning. The first interview established the context of the participants' experiences. The second interview allowed each participant to construct the details of his/her experience within the context in which it occurred, and the third interview encouraged the participant to reflect on the meaning his/her experience holds for them. Figure 4 Illustrates Seidman's Three Stage Interview Process that the researcher utilized during the data collection process. 


\section{1st Interview: Background Information Relationship}

Building

-Establishes the context of the participants' experience

- Gather Background information

\section{2nd Interview: Construction}

- Construct the details of his/her experience within the context in

\section{3rd Interview: Reflection}

-Participant reflects on the meaning his/her experience holds for

him/her

- Validate Experience

Figure 5. Seidman's Three Stage Interview Process Used in the Data Collection Process

During the interviews, it was important for the researcher to take extensive notes and to process and clarify where necessary. The handwritten notes included observable behaviors that could not be tape-recorded during the interview process, such as body language, intonation, and gestures. Extensive note taking validated the experiences by the participants' behaviors or gestures. The researcher integrated Kvale's seven-stage interview process with Seidman's three-stage interview process to collect, transcribe, analyze, and report the data during the data collection process over a period of 4 months. Thematizing, designing, the study, and developing the interview guide are the first three steps in Kvale's seven-stage interview process. 


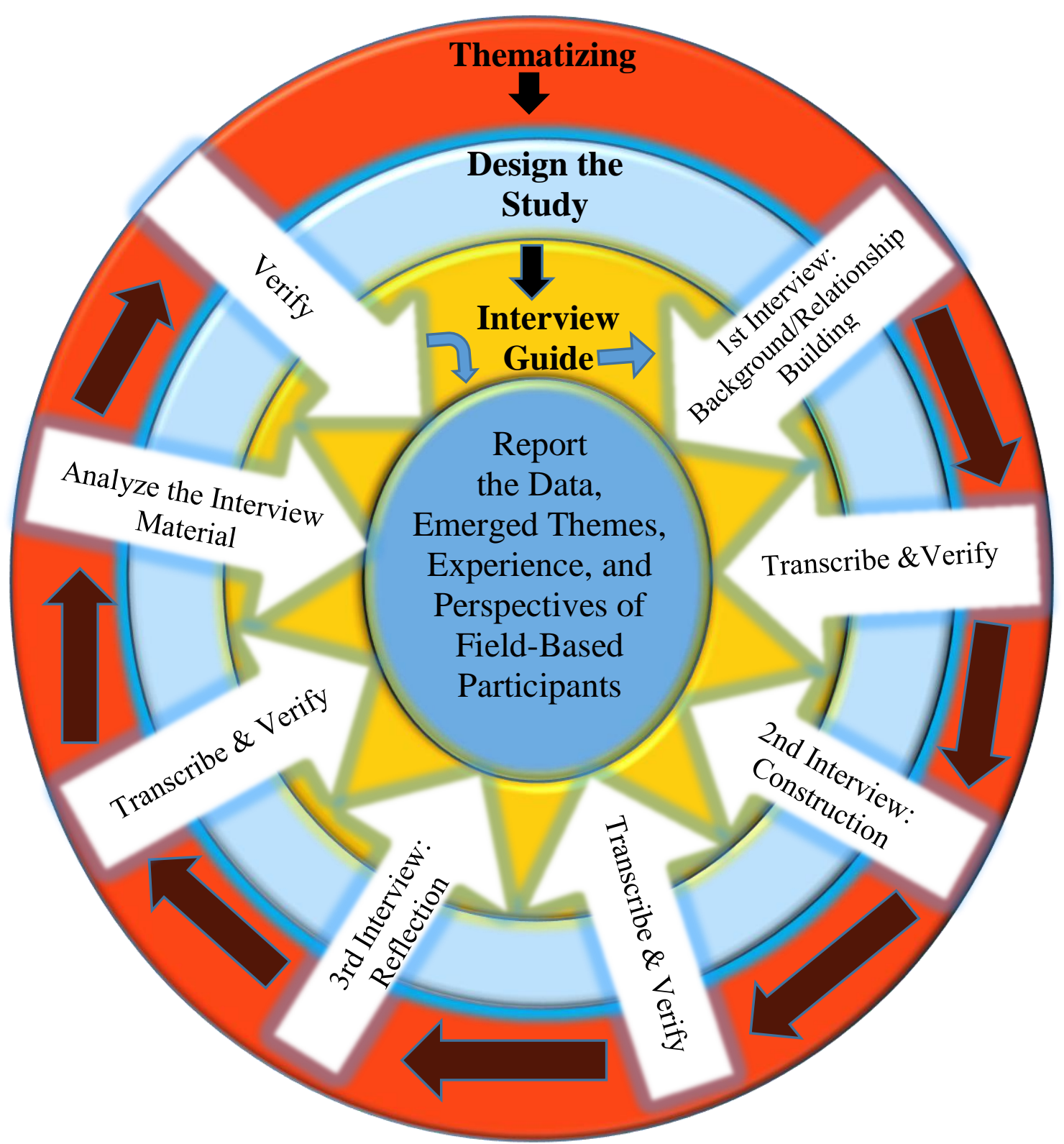

Figure 6. Kvale's Seven Stage and Seidman's Three Stage Integrated Interview Process

The first two of Kvale's seven-stage interview processes were completed in

Chapters I and II of this research study. The remaining stages took place during and after 
the actual interview process. Next, the researcher incorporated three separate interviews for each participant following Seidman's three-stage interview process. Each interview lasted approximately 90 minutes. After the data was transcribed, the researcher verified the data by emailing the document to the participant to verify the accuracy and meaning as told by the field-based participant. The aforementioned interview procedures were repeated until the third interview was transcribed and verified. Next, the researcher analyzed all of the data to identify emerging themes and significant details. Then, the researcher verified the experiences and perceptions, constructs and (co)constructs meaning of the analyzed data. Last, the researcher reported the data.

\section{Data Collection}

Initial contact was made with the field-based coordinator of the inner city fieldbased teacher preparation program (INC/FBTC) via email to secure the university alumni book directory and a list of the 1994-2013 field-based participants. The INC/FBTC coordinator assisted in securing the email addresses of the participants between 1994 and 2013. The coordinator of the IN/ FBTP participants emailed a letter of introduction that informed the participants of the research topic and a request for their participation in the research. The data collection process began during February 2014. Between 1994 and 2013, 16 participants responded, indicating that they would participate in the study. However, only four participants scheduled an interview session for the study. Transcription began immediately after conducting the interview. The transcriptions were sent to the participants for verification the next day, and each of the participants responded within one month. The researcher also scheduled the next meeting upon 
verification of the data in the transcriptions. Table 3 illustrates the actual number of participants in the study, their ethnic backgrounds, their gender, a timeline of the data collection process, transcription dates, and the dates when the data was validated by the participants of the study.

Table 3

Interview Timeline of Data Collection Process

\begin{tabular}{lcccc}
\hline Interviews & Participant \#1 & Participant \#2 & Participant \#3 & Participant \#4 \\
\hline Gender & $\mathrm{F}$ & $\mathrm{F}$ & $\mathrm{F}$ & $\mathrm{F}$ \\
Ethnicity & African & African & African & African \\
& American & American & American & American \\
Initial Contact & $1 / 24$ & $1 / 24$ & $1 / 24$ & $1 / 24$ \\
Interview \#1 & $2 / 9$ & $2 / 16$ & $2 / 27$ & $2 / 24$ \\
Transcription \#1 & $2 / 9$ & $2 / 16$ & $2 / 27$ & $2 / 24$ \\
Validation \#1 & $2 / 17$ & $2 / 23$ & $3 / 3$ & $2 / 27$ \\
Interview \#2 & $3 / 25$ & $3 / 22$ & $3 / 29$ & $3 / 27$ \\
Transcription \#2 & $3 / 25$ & $3 / 22$ & $3 / 29$ & $3 / 27$ \\
Validation \#2 & $3 / 31$ & $3 / 25$ & $4 / 3$ & $3 / 30$ \\
Interview \#3 & $4 / 13$ & $4 / 14$ & $4 / 6$ & $4 / 13$ \\
Transcription \#3 & $4 / 13$ & $4 / 14$ & $4 / 6$ & $4 / 13$ \\
Validation \#3 & $4 / 15$ & $4 / 18$ & $4 / 13$ & $4 / 21$ \\
\hline
\end{tabular}




\section{Data Analysis}

Research of this type generated a large quantity of data that was transcribed and analyzed. The transcribed data was juxtaposed, analyzed, and compared to identify the quintessence of the phenomenon, for example, the essence of having a mentor, the essence of developing relationships, or the essence of being a participant in the program. Denzin and Lincoln (2000) state that qualitative research involves the use and collection of a variety of practical materials, case studies, personal experiences, life stories, interviews, artifacts, cultural texts and productions, and observational, historical, interactional, and visual texts, all of which describe habit and challenging experiences and meanings in individual lives. Using the personal experiences, gathering background information and interviews aided in making sense of the field-based program without imposing preexisting expectations on the participants being researched, which consists of a set of interpretive material practices, positioned to identify the observer during the data collection process. Hence, such practices transformed the data collection process into a cycle of representations, including observation notes, interviews, conversations, recordings, and memos (Creswell, 2012).

Analyzing the data and identifying themes required a great deal of time. Creswell (2005) defines themes as smaller codes aggregated together to form a major idea in the database. During analysis, it was important to examine themes as they emerged during the data analysis and limit the analysis to five to seven major themes. Creswell (2005) states there are several types of themes, but the researcher must use the data to identify themes. A small-scale analysis was used for this study across themes and among 
participants because it allowed the researcher to input data under the various theme headings that were juxtaposed. During the three interviews, the dialogue between the participant and the researcher was analyzed, starting from the past to the present experience in the field-based teacher preparation program.

\section{Saturation}

When implementing the phenomenology research method, saturation was necessary to identify the emerging themes to build layers of intricate analysis from the interview data, particularly with a small sample of data that was interpreted in its entirety and focused on experiences. Seidman (2013) suggests that in-depth interviewing populates a vast amount of data. Although only four participants were interviewed, a large amount of data was generated as a result of the three interviews. As the data was read, the researcher identified themes, made notes on the side column of the paper, and used post-it paper for each theme that emerged from the data. Next, the themes were

placed in one row on a floor and on the wall. The data was then cut into separate sections and placed under each theme. This same process was performed for each interview for each of the four graduates. By constantly working and reworking the data and reviewing it in multiple ways, saturation occurred.

\section{Juxtaposition}

Juxtaposition involved interviewing a small sample of participants where the interpretation was co-constructed between the researcher and the participant. The researcher implemented juxtaposition to interpret the participants' perspectives of their field experience. Grbich (2013) explains that the focus during juxtaposition is on the 
complexity of the experience and language, specifically, the researcher focused on double coding the entire conversation and identifying how the language was communicated and deconstructed.

\section{Reliability and Validity}

Ascertaining the reliability and validity of the data was very important. Critical to this research was articulating the procedures that were followed to ensure accuracy and reliability. Reliability and validity is a vital component in determining the suitability of the instrument in the data collection process (Mertens, 2005). Collectively, reliability and validity informs the researcher if the instrument properly evaluates what it was intended to evaluate and determines the truth worthiness of the research (Kvale, 2007, Creswell, 2007, Seidman, 2006; Patton, 2002). Reliability and validity provide avenues to indicate how the researcher constructed meaning of the experience in the context of the research phenomena being analyzed (Grbich, 2013). For the purpose of this research, methods such as detailed descriptions, checking, and clarifying biases were selected to assure the implementation of reliability and validity.

\section{Reliability}

Kvale (2007) defines reliability as the consistency and trustworthiness of research. He suggests that the researcher should question if the data is reproducible by others at different times. Other researchers add that reliability is the approach in which the researcher demonstrates consistency within the research (Trochim \& Donnelly 2010; Kvale, 2007; Creswell, 2007; Seidman, 2006; Patton, 2002). Schwartz-Shea and Yanow (2012) affirms that reliability assesses the extent or measurement to determine the 
trustworthiness of any research. In understanding reliability, the researcher explained any discrepancies in the study and checked for consistency among the three interviews (Saunders, Lewis, \& Thornhill, 2009; Kvale, 2007; Fink, 2008).

The researcher confirmed that the data was that of the researcher without interruption or influence. Gibbs (2007) asserts that the researcher's approach is consistent across different researchers and projects. Critical to this research was the ability to insure reliability through the research process. The researcher provided a detailed explanation for the focus of the study and the participants' positions, the basis for their selection, and the context from which data was collected. For the purposes of this study, the researcher implemented the aforementioned procedures to insure reliability.

\section{Transcriptions}

In terms of reliability, the researcher transcribed the language from the participants' interviews and listened to the audio to verify that the participants' transcripts were accurate. The researcher listened to the audio tape more than once to identify possible discrepancies due to poor audio, noise background, and participants' speaking low.

\section{Coding}

After transcribing the data, the researcher reviewed each line of text, used the language from the participants' interviews to develop the codes and themes, and then assigned codes. The researcher continuously reviewed and compared the data and codes generated by maintaining a daily log of codes. Codes were then either recoded or 
combined where necessary. This ensured consistency with coding throughout the interviews and the data analysis process.

\section{Crosschecking}

The researcher used another person to cross check the codes or compare results from the research to the codes developed by other researchers. Qualitative research incorporates a number of techniques to ensure reliability. Three techniques were used to ensure reliability. First, the researcher provided a detailed explanation for the focus of the study and the participants' position, the basis for their selection, and the context from which data was gathered (Creswell, 2013). In addition, data collection and analysis strategies were reported in detail in order to provide a clear and accurate picture of the method used in this study.

\section{Validity}

Many researchers define validity as a data collection process performed by two or more researchers collaborating on the research that alludes to the experience or interpretation discussed in the inquiry and a method of asking questions (Schwartz-Shea \& Yanow, 2012; Creswell, 2012; Kvale, 2007; Behling \& Law, 2006; Strugwiz \& Stead, 2004; Trochim \& Donnelly, 2006). It refers to the truthfulness and accuracy of the research and the degree to which the method explores what it intended to research (Creswell, 2007; Seidman, 2006; Patton, 2002). The Standards for Educational and Psychological Testing American Educational Research Association (AERA) (2014) suggests that validity is a perception that analyzes the degree to which all gathered data supports the intended interpretation or purpose. 
In the construct of validity, this phenomenological research study analyzed the experiences and perceptions of graduates from an INC/FBTP. Seidman's (2006) three stage interview processes utilize procedures that enhance the attainment of validity. Additionally, these interviews afford participants an opportunity to make sense of their personal experiences and their comments in perspective of the research. Above all, the researcher was able to make connections among the experiences and checked for consistency in what was said by connecting the participants' experiences and comparing the three interviews. Validity is apparent if there is evidence of concern for the participants' understanding and authenticity of what the participants are saying (Trochim \& Donnelly 2010; Kvale, 2007; Creswell, 2003). The researcher implemented the following procedures to check for the validity of the findings.

\section{Transcription}

In terms of validity, the researcher used a digital audio recorder to record the interviews and took every precaution to ensure accurate transcriptions of the data as told by the participants. Transcription involves translating the oral experiences of the participants from an oral language to a written language. Transcripts are interpretative constructions from the researcher and participant. Kvale (2007) suggests it is important to recognize the tone of voice, pauses, and repetitions when interpreting or transcribing the data. This highlights the nuances of the field-based program and develops meaning to the participants' meanings. 


\section{Member Checking}

The researcher conducted a follow-up interview after the first and second interviews to verify the accuracy of the generated transcriptions, explanations, interpretations, coconstruction of the meanings and themes. Huberman, Miles, and Saldana (2014) indicate that member checking can enhance the quality of both the data and the final conclusion. Additionally, member checking assists in "verifying or extend interpretations and conclusions helps with anonymity problems (p. 63).

\section{Description}

The researcher encouraged the participants to provide a very clear and detailed description of their experience (data collection and analysis strategies). The data reported as told by the participants allowed for the emerging themes. The focus was on the distinct descriptions that illustrate the various levels of multiplicity, the differences, and varieties of the field-based teacher preparation program. Additionally, data collection and analysis strategies were reported in detail in order to provide a clear and accurate picture of the method used in this study.

\section{Clarifying Bias}

Data analysis and reporting must be free from biases during the validation process. Reflecting on the biases communicates how the researcher's data interpretation is influenced by prior experiences (Creswell 2007; Kvale, 2007). As one of the first graduates from an INC/FBTP, the researcher brought prior experiences to the study; however, those experiences did not skew the research because each participant owned their respective experience. No one experience is the same. Recognizing these biases 
illuminated specific aspects of the research study and added new facets to the study, which added to the constructed perspectives. It was necessary that the researcher clearly communicate and clarify any and all biases.

\section{Adverse Data}

Daily life experiences consist of both negative and positive experiences.

Reporting both positive and negative data served as a valuable tool to the researcher. Creswell (2007) suggests that communicating these experiences adds credibility and validity to the experiences.

\section{Time}

The researcher conducted three interviews and spent approximately 90 minutes with the participants during each interview to develop an understanding of the in-depth interviews and assist in conveying details about their experiences (Kvale, 2007; Rapley, 2007).

\section{Internal Validity}

Seidman (2006) maintains that the three-interview process interprets the participants' interviews in context and accounts for idiosyncratic ways to check for the internal consistency of what the participants communicated. Internal validity occurred after the first interview and continued through the third interview. The researcher listened to what the participants said, observed the body language of the participants, and analyzed the data from the first interview. Next, the researcher created follow-up questions for the second interview to clarify, where necessary. Internal validity was 
confirmed when the researcher asked participants to reflect on their field-based experience and to reconstruct the experience.

\section{Ethical Considerations}

Ethical limits are a judicious component of any form of research. All of the participants were treated in accordance to the ethical guidelines of the American Psychological Association (APA) and Institutional Review Board (IRB). Although risks were associated with participation in the study, a few considerations were kept in mind when engaged with the participants, designing, and analyzing the study. First, all of the participants were interviewed in a setting that was most comfortable and selected by them. During the first interview session, each participant was given an informed consent form to sign. Secondly, there was a possibility that the participants might experience a level of stress or discomfort discussing their experience in the schools, their peers, or their relationships with professors and colleagues. All of these considerations were incorporated in the research design. Every precaution was taken to ensure the participants felt comfortable and clearly understood that they had the freedom to withdraw from the research study at any time.

Two possible types of risk were associated with this research study: during the interview and after the completion of the three interviews. Whenever data is confidential, there is always a risk of breach of confidentiality and a possibility that the participant could be identified as a participant of this study. During the interviews, all of the participants reflected on experiences that they found unpleasant or upsetting and comforting. The process of interviewing caused a level of discomfort at times. The 
researcher worked to minimize discomfort, and pseudonyms were substituted in the final report for all names of persons, schools, and city to disguise the participants' identity. Every step was taken to disguise the participants in this study.

\section{Limitations of the Methodology}

Limitations for this research included: (a) a small sample size, (b) all of the participants being from the same program but different years of participation, and (c) the use of interviewing as a method of collection data. The researcher used a small sample of four students who participated in, and later graduated from, an inner-city field-based teacher-preparation program, which limited the research from generalizing the data. Limiting the sample to four participants in the three interviews generated a large amount of data (Lester, 2012), which allowed the researcher to interpret data more independently. It was necessary for the researcher to focus on interpreting the oral experiences and perceptions as articulated from graduates of a field-based teacher-preparation program into written text.

The researcher's familiarity with the field-based teacher preparation existed from personal participation and graduation from such a program. Personal biases while collecting and analyzing data provided structures that shaped perspectives of the program and the teaching profession. More importantly, the researcher's prior experiences were different from the other participants because the researcher traveled abroad to conduct research and participate in a field-based program for 6 months. Additionally, the participants were assigned to different schools each semester versus one selected school with different academic majors. 
Kvale (1996) explains Gadamer's theory of interpretivism and maintains that a person's preconception can hinder the interpretive process. The researcher must actively analyze what educators bring to the text and what the text brings to educators. An important aspect of Gadamer's (1960/1990) theory suggests that during interpretivism, the researcher must distinguish between the meaning of the text and understanding the person (Kvale, 1996).

Traditional field observations were not part of this data collection process. However, the researcher observed the nonverbal cues of the participants during the interview. This was necessary to determine consistency and validity of the experiences as told by the participants.

Time and availability were critical during the data collection process. Miles, Huberman and Saldana (2014) proposes that time allows the researcher to develop a relationship with the participants of the study. Enough time was scheduled to allow the participants to speak openly without feeling that they were pressed for time or could not speak freely. Unforeseen circumstances intercepted the scheduled time of the interviews, such as traffic, last minute school meetings, and location of interview hours of operation changed. The participants' availability varied depending upon the start and end time of their school, and if they participated in afterschool activities. All of the participants requested to be interviewed during the weekend for the first interview. The participants then requested to meet during the week to finish the data collection process. All four of the participants were working in the teaching profession and pursuing their master's or 
doctoral degrees. The researchers' time was flexible to accommodate the availability of the participants. The researcher made sure there was adequate time to interview the participants and interpret the data for the study.

Location was critical to the data collection procedure because the location dictated the time the participants would be available. The participants identified locations that were feasible to them and close to the schedule for the dates of the interviews. Location was important for interviews because the participants had to feel comfortable to participate in the interviews and trust that the place would not disclose their participation in the study. The participants selected each of the locations and times.

\section{Conclusion}

The purpose of this study was to focus on the experiences and perceptions of university graduates who participated in an INC/FBTP. To accomplish this, a qualitative methodology was used as a means to gain insights and to interpret the perceptions and experiences of the participants who graduated from such programs. Phenomenology was used as a method to gain an in-depth understanding of the meaning of the experiences for the participants. This chapter detailed how phenomenology was used as a method to conduct structured and semi-structured in-depth interviews, collect data, and analyze data. In-depth interviewing incorporated open-ended questions, three stages of structured and unstructured interviews, planning, and preparation (Seidman, 2006).

In this chapter, the researcher provided a clear explanation for identifying themes and codes from the interviews to gain insight and understanding into the lived experiences of the graduates who participated in an INC/FBTP. An explanation of why 
phenomenology was appropriate for this research design, the participants, limitations, biases, and ethical issues were discussed. 


\section{CHAPTER IV}

\section{ANALYSIS OF RESEARCH}

While previous research discussed field-based perceptions, no research incorporated the experiences and perceptions of participants until this study. The purpose of this study was to explore the perceptions and experiences of graduates from an INC/FBTP. The study sought to answer the following overarching question: What are the perceptions and experiences of participants in an INC/FBTP regarding their preparedness in terms of: (a) perception of preparedness; (b) knowledge, skills, and attitudes critical for participants' success; and (c) relationships the participants developed with professors and mentors.

The results of this phenomenological data analysis are presented in the form of theme descriptions of the participants' experiences. This chapter is divided into three main sections: university and participant backgrounds, developed themes, and summary of findings. The inner city field-based university background information was generated from the demographic and socioeconomic data available to the public. The participants' background profiles were generated using the participants' own words via the interview processes of Seidman (2013) and Kvale (2012). The interview process focused on the meaning the participants made of their experiences. After reading all of the participants' backgrounds and interview responses, the experiences were synthesized, which allowed 
for both researcher and readers to comprehend the social factors that contributed to influencing the participants' field-based experience. The participants are introduced in the order in which the interviews were conducted. Each participant and the inner city university were assigned pseudonyms to assure their identities were protected.

This chapter presents developed themes from the data analysis process. Each developed theme is supported by sub-themes that were generated from the data analysis. Ultimately, these themes and sub-themes provided insights into the experiences and perceptions of each participant as it related to their preparedness for the teaching profession. The second section presents the summary of the findings.

Three out of the four participants (Sharon, Yolanda, and LaTonya) reported that they gained experience as a teacher in other career fields prior to engaging in the program. After the completion of their respective field-based program, three of the participants reported at least 5 years or more of experience as a professional working in the educational field. Participants' majors included elementary education, secondary education, business education, and higher education.

The subjects in this study participated in a 2-year field-based teacher preparation program at two or more schools in the inner city teaching in elementary, middle, and high schools. According to the participants, the field-based teacher preparation schools were located within the minimum 10 minutes ( 2 miles) to 1 hour ( 22 miles) from the home university. Participants traveled to their school locations by car, bus, or metro. Partner schools in the Field Based Teacher Preparation Program were located anywhere from 5 to 25 miles away from the university location, and all schools were located in a large urban 
environment with a high crime rate. Schools were diverse, but the specific percentages of the various ethnicities of each school are unknown. In addition, the schools had a high percentage of students eligible for free and reduced lunch. Only two of the five partner schools were meeting Annual Yearly Progress (AYP) at the time of their participation in the program. Figure 7 illustrates the location in miles and street numbers of the inner-city field-based school placements.

Two participants were paired to a school, but each participant was placed in a different grade level at the given school. Each semester, participants were placed in a different grade level, so within the 2-year program, each participant was placed in two grade levels in two different schools, four grade levels overall. Three of the four participants indicated that part of their curriculum required engaging in volunteer opportunities within the inner city and community. All four of the participants were actively engaged in the field-based program and successfully graduated from an INC/FBTP.

Three of the four participants successfully obtained their teacher certification, and all of the participants were working as educators in an academic setting at the time of this study. Two of the participants were classroom teachers and had taught longer than 5 years after the experience, and the other two participants left the teaching in a selfcontained classroom within the first 5 years of working in the teaching profession, but currently worked in another educational career field.

The data presented is internally consistent among the three interviews. The interviews were conducted within 2-3 weeks apart. Sideman (2013) suggests internal 
consistency develops during a length of time and adds validity to what is being said by the participant. During the interview process, a number of observed behaviors among the participants included long and short pauses, loud and soft laughter, crying, staring out of the window, smiling, and waving hands over the table. This behavior verified that the participants pondered seriously with questions as to the value of the program as it related to their perception and preparedness as first year teachers and added validity to the experience as told by the participants during that time. The interactions between the researcher and the participants in terms of understanding the social context of their experience of and lived realities (the assigned school, mentor teachers, parents, and students), provide an understanding of participants' experiences and draw on previously acquired cultural knowledge. As a result, assumptions about the field-based teacher preparation program can also be understood. Socially, field-based participants are placed in neighborhood schools that may differ from their own, which potentially fosters meaningful and effective field experiences within the school community.

\section{Developed Themes}

The following themes and 19 sub-themes became evident when discussing the experience with the four participants interviewed. The following themes developed, and definitions were generated from the interview data. The significant details are the topics that were discussed by the participants that were important to discuss but did not have the same level of importance. The participants indicated that the significant details were important in their development. Table 4 illustrates eight themes and definitions that developed from the responses from the interview questions. Additionally, the table 
presents the 19 sub-themes themes and 11 significant details that correlate with the themes that emerged during the data line by line coding and analysis process.

The next section is a discussion of the first theme and significant details that developed during the data analysis process titled "Perception." Sub-themes discussed in this section include daily challenges that the participants discussed that influenced their perception of the program. Some of the daily challenges discussed by all of the participants included transportation to the assigned schools, community service projects, personality conflicts, and financial strains.

Table 4

Themes, Sub-themes, and Significant Details

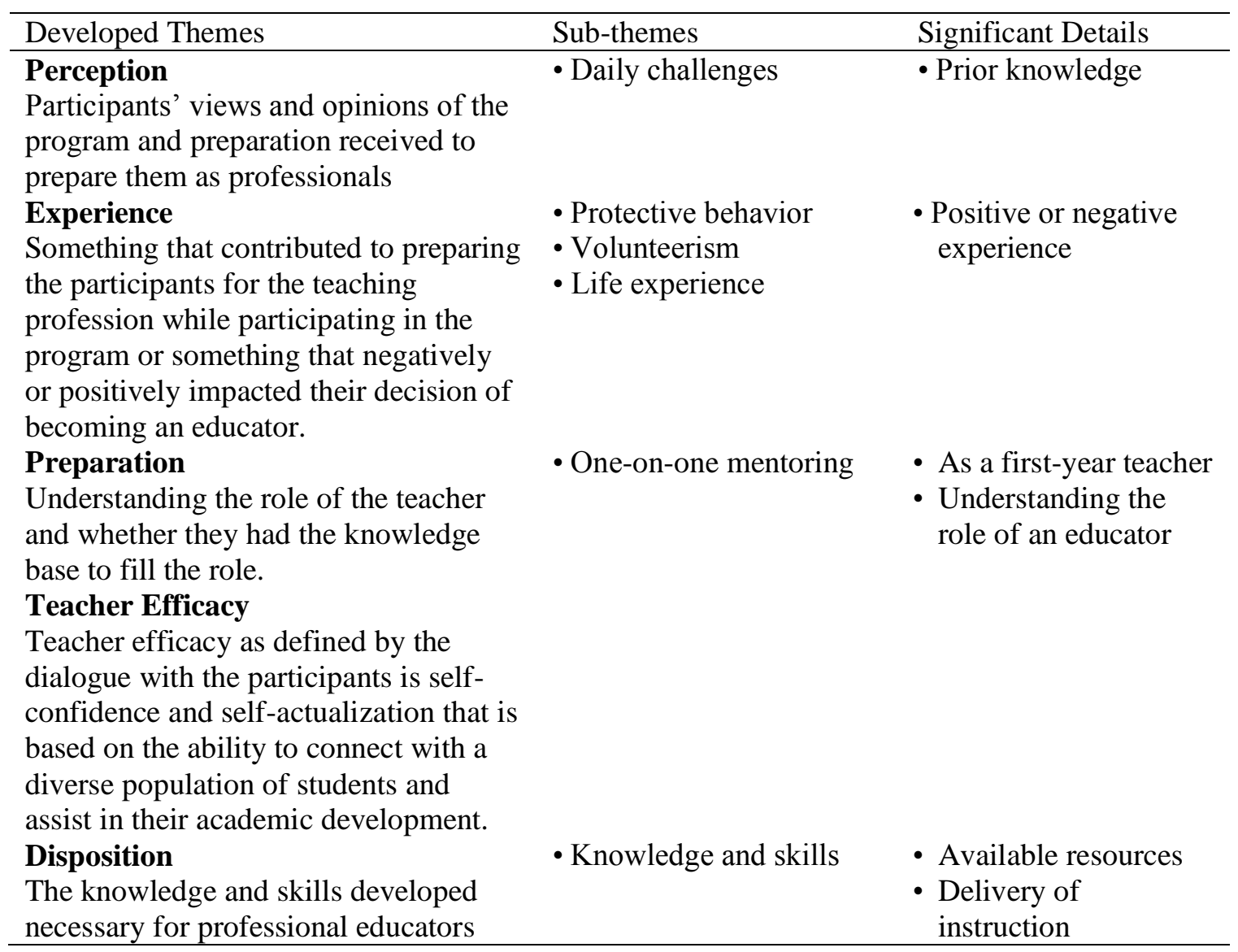




\begin{tabular}{|c|c|c|}
\hline $\begin{array}{l}\text { Collaborative Relationships } \\
\text { The ability to effectively develop } \\
\text { relationships and communicate with the } \\
\text { program community }\end{array}$ & $\begin{array}{l}\text { - Professional } \\
\text { relationships } \\
\text { - Coordinator } \\
\text { - Trusting relationships } \\
\text { - Participants' strengths } \\
\text { and weaknesses }\end{array}$ & \\
\hline $\begin{array}{l}\text { Program } \\
\text { The nuances of the program } \\
\text { curriculum, professors, participating } \\
\text { schools and participants and } \\
\text { coordinator }\end{array}$ & $\begin{array}{l}\text { - Strengths } \\
\text { - Weaknesses } \\
\text { - Strengths } \\
\text { - Weaknesses }\end{array}$ & $\begin{array}{l}\text { - Leadership } \\
{ }^{\text {a }} \text { Financial resources } \\
\text { - Prepared participants } \\
\text { - Lack of participating } \\
\text { schools } \\
\text { - Lack of university } \\
\text { support }\end{array}$ \\
\hline $\begin{array}{l}\text { Recommendations } \\
\text { Suggestions to recruit participants and } \\
\text { improve collegiality between the } \\
\text { participants, university, and school } \\
\text { mentors }\end{array}$ & $\begin{array}{l}\text { - Financial } \\
\text { - Expansion } \\
\text { - University support } \\
\text { - Professional } \\
\text { development } \\
\text { - Feedback } \\
\text { - Record keeping } \\
\text { - Curriculum }\end{array}$ & \\
\hline
\end{tabular}

\section{Perception}

Perception was defined as the views and opinions of the program and preparation received to prepare the participant as first year teachers. The participants perceived that a number of factors contributed to and highly influenced their success as a first year teacher and learner. Some of the factors that were discussed included prior knowledge, experience, course load, interactions with other participants and community. The prior knowledge that the participants brought to the program influenced their peer collaboration, learning, peer interactions, and perceptions of the teaching profession while in the program. 


\section{Daily Challenges}

All of the participants indicated that they perceived participation in the program as eliminating some of the daily challenges of traveling to the university to attend class on campus, maintaining employment, and personal issues. However, the participants still had difficulty in balancing school expectations with their personal lives, the demands of the field-based program, and completing the required program coursework. This was evident in her conversation, when Lucretia said, "We had full-time jobs, and had to find financial resources to travel to the sites and still complete the program course work."

Most of the participants realized that the teaching profession was not a glorified profession. Many times educators have to deal with personality conflicts and daily events constantly changing. Most importantly, educators have to be flexible and ready for anything to happen. These experiences played a major role in their decision to seek or not seek employment in the teaching profession. Sharon stated, "It gave me a sense of awareness of the teaching profession. My perception of the program was like this is incredible because this is what you do in a real world." However, another participant indicated that being placed in various communities heavily influenced her perception of the teaching profession. Lucretia said, "My perception was enhanced by being placed in schools that were located in various low socioeconomic communities." Yolanda said, "This experience would help me interact with the inner city students, and I was not afraid of being in the community as many of my peers in the program." This experience was an 
opportunity for her to understand and connect with the community elements and the students.

LaTonya indicated that the program changed her perception of the teaching profession and heavily influenced her decision not to pursue a teaching career. She said, "I can deal with one or two of them in the office but 30 in the classroom. No! They are on high energy and they are not going to curse me out!" This was the turning point for LaTonya; she said, "No, the classroom is not really for me." This experience negatively altered her perception of the teaching profession and the students in the classroom that she would later teach. This was unique to Yolanda because of the experiences she had in two of her assigned schools. Latonya stated that having a time to reflect and share with the other participants and professors in the program developed her ability to problem solve and provided her with a wealth of advice that developed her self-confidence and ability to handle various classroom situations.

\section{Experience}

The second theme that developed from the data analysis was "experience." For the purpose of this research, experience was an element that contributed to preparing the participants for the teaching profession while participating in the program. Analysis of the participants' experiences revealed a number of similarities and differences. Similarities included the amount of time spent in the field, the school community, the wealth of knowledge and skills available to them, the on-going support from the coordinator, and the life experiences gained from their assigned schools and volunteering in the community. However, there were some notable differences experienced among the 
participants in the program, program requirements, and relationships with the assigned school mentor and supervising teachers, relationships with university professors and the classroom experiences. For Lucretia, the program had a limited number of participating schools. In addition, while she was in the program, two participants were awarded scholarships to either study abroad or at another university. The field-based program allowed one of the participants to travel to Minnesota and the other one to travel to Japan to conduct educational research. Additionally, Lucretia indicated that the program had a great deal of university and college support from the assistant dean and the dean of the College of Education. The remaining participants indicated that their program had a wide selection of participating schools, an optional study abroad component, and required community service, but the program lacked university and college support to continue to promote the program.

All of the participants indicated that they had a very positive experience from the first day, including meeting the program coordinator and learning about the dynamics of the field-based program. They spoke highly about the relationships that developed, including the way the coordinator approached and communicated with potential participants and the availability of resources to the participants as one of the most important aspects of the program. They were impressed with the level of professionalism modeled by the coordinator and the attentiveness to the needs of the students, and they indicated that these were key factors in recruiting education majors into the program.

Yolanda described her first impression and experience with the program coordinator before entering the program, when she was having difficulty with one of the 
deans in the college of education. She learned that the university had a program that was "connected to her program" of interest and she wanted to learn more about the program. She overheard the coordinator answering questions for another student interested in the field-based program. At the time, Yolanda was not involved in the program. Therefore, she decided to wait and listen to gather more information. She was impressed with the coordinator because the coordinator explained that she was busy at the present time but would be "available to speak with her in 30 minutes." After speaking with the coordinator and receiving valuable information about the program, Yolanda indicated that she then enrolled in the program because she wanted a program that had a "school and university connection." She said, "I could not imagine what I would have done had she not been as involved as I was."

LaTonya shared how the interactions with the program coordinator resulted in her developing self-confidence and reaching out to the younger participants in the program. It was evident from the participants' comments that the coordinator modeled the behaviors that were expected of all of them. LaTonya said, "She made herself readily available to assist the participants and had an open door policy to encourage participants engage in dialogue with her."

Interactions with the coordinator and mentors included actions and events that were directly connected to the teaching profession, rules and regulations, communication, and field-based decisions. The daily social interactions in the program provided unique experiences, such as volunteering in the community, developing relationships with the mentors, peers, professors, and program coordinator, all of which empowered the 
participants and assisted in their development as professional educators. The community in this sense is the field-based teacher preparation program, the participating schools, and the school community. For example, when asked to describe her experiences in the fieldbased teacher preparation program, Lucretia indicated that she was one of the original participants in the first field-based teacher preparation program, one of the first graduates from the program, and the program was the first of its kind at the university and in the community. She said, "I was pleased because I was placed in real classrooms with real experiences instead of a school that was a cookie cutter school where everything was perfect." Lucretia appreciated having the opportunity of being placed in a school where nothing was staged or hidden from her experiencing the impromptu classroom events and realities of the teaching profession. LaTonya said her experience allowed her to see the students being disrespectful, using profane language, and not having materials for class. "I was able to see everything that I needed, and that I would experience in a room, in a real life setting as a new and incoming teacher." This experience allowed Lucretia to identify ways she could connect with the students by engaging in daily conversations.

Sharon indicated that the program taught her "how to deal with people, a kid crying, a parent or someone is unhappy, and hands-on experience in different environments." Yolanda shared her experience with the inner city university faculty and staff when she questioned her role as a student and how her teaching program would connect her to the community. "I really didn't have a good experience at the university." Sharon shared that her interactions with some of the professors and mentor teachers were unprofessional and that she was not happy with the interactions while in the program, 
more specifically, the interaction with the home university, when attempting to gather information regarding certification or financial assistance.

\section{Protective Behavior}

The next sub-theme developed was protective behavior that was modeled by the on-site mentors and school staff toward the field-based participant. LaTonya developed a sense of respect and appreciation for the staff because of their display of respect for her, protective behavior, and the professionalism of the school staff. LaTonya indicated that the mentor teacher would allow her to leave immediately after the bell rang because the individual did want her at the school late. The mentor teacher indicated to LaTonya that the neighborhood was high-crime, gang infested, and that he did not want anything to happen to her. This level of ethical leadership style demonstrates care for others, focuses on truth, and selfless aims.

LaTonya validated the protective behavior demonstrated by the school staff. She indicated that she was placed in two different schools and the school staff was very protective of her. She said, "The mentor teacher told me that the school staff was protecting me and that they did not want me to get involved in anything over there [her second school placement]." According to Latonya, the neighborhood was drug and gang infested and that it was not safe during the evening hours at the school. Yolanda indicated that the field-based program coordinator was protective of everyone in the program daily. She explained that the coordinator said to her, "Choose her battles; if it didn't pertain to her classroom don't get involved." Yolanda was troubled with the participants' affairs and what was going on at the university. She felt that these behaviors were impeding her 
performance in the program. The coordinator worked collaboratively with the participant to develop her ability to focus on her professional development in the program, and she made sure that she met the program requirements. Lucretia indicated that the assistant dean and the dean of the College of Education were equally protective of all of the participants in the program and wanted all of them to be successful. Lucretia said the assistant dean would always find time to ask if "we needed anything?" They showed compassion and at the same time gave tough love. She indicated the one thing that stood out the most to her was that the academic field-based team were always dressed professionally and conducted themselves professionally.

\section{Positive or Negative Experience}

Participating in the field-based teacher preparation program not only provided the participants with first-hand authentic classroom experience, but experience with work related and personality conflicts among practicing teachers in the field. Two of the participants explained they had to overcome adverse situations with teachers feeling uneasy with the field-based participants in the school. All of the participants indicated developing positive working relationships with the tenured teachers was very important in the workplace. LaTonya indicated that she engaged in a conversation with one of the teachers to assure her that as a field-based participant, she was not trying to replace her on the job. Lucretia indicated that she had to step back a little and not appear as knowledgeable as the teacher to gain the respect and acceptance of the other teachers. Last, the participants indicated that they engaged in many conversations to build positive relationships and to assure that the tenured teachers were comfortable with the field- 
based participants. Experiences are very important regardless whether they are positive or negative. The interpretation of positive or negative experiences varies depending on how the person defined the experience.

LaTonya indicated that her field-based schools failed to provide her any form of in-depth experience and that it was an opportunity for her to perform observations and volunteer services. She indicated that the "majority of her mentoring came from the fieldbased professors and coordinator" while in the program, which made her "a little bit more prepared and astute for the classroom." This level of mentoring, in her opinion, prepared her as a participant and caused a level of friction in some of the schools where she was placed. She said, "they caused problems for me and I was told to be selective in my dialogue with the teachers because I didn't want to appear more knowledgeable than the supervising teachers." As a result, LaTonya felt that the "experience wasn't a good experience" because she interpreted the conversation as being asked to "hold back from being herself and teaching." Additionally, LaTonya indicated failing to adhere to the advice of the professor could ruin her opportunity of completing the program. She said, "The university professor could give me a low observation rating and that could ruin or jeopardize my score or chance of completing the program."

Yolanda indicated that she had a positive experience because the program taught her not to be "afraid to ask questions." She realized that she had reservations about questioning things that she did not understand but challenged herself to ask more questions, because she felt that was "the only way she was going to learn." She also said the programs helped her to assess her personality because she realized it was difficult for 
her to refrain from engaging in dialogue. Yolanda said, "I wasn't going to keep my mouth shut. Thank God, I changed. So, I learned one thing: if I didn't understand, ask and that is the only way you are going to learn."

\section{Volunteerism}

Although experiences varied among the participants, volunteering was one of the experiences that continued to surface during the interviews. Volunteering proved to be a critical component in the development of teachers. It provided an opportunity for the field-based participants to connect with the school community, network with future employers, develop, and strengthen social skills through their shared field-based activities. Volunteering activities for these participants included volunteering for the university campus and community functions, passing out flyers, and volunteering at the various museums. Volunteering in the community not only empowered the participants, but it exposed the participants to the culture and language of the program that developed their understanding of the teaching profession. Participants were able to comprehend the social and economic dynamics of the students in their classrooms.

Lucretia expressed her joy as a volunteer with the field-based program. Her experience began early in the program with the coordinator and continued through the duration of the program as a volunteer. "The person running the program was very impressionable and she required that all participants perform volunteer work." Her first volunteer assignment was the alumni picnic, where she assisted with "setting up for the picnic, creating and passed out flyers." Another volunteer experience included 
volunteering in one of the museums where she volunteered as a tour guide and facilitated student and teacher workshops.

Participants believed that the amount of time spent in the field volunteering provided them an opportunity to develop the specific professional skills that would enable them to secure employment as a professional teacher. These skills included effective communication skills, interviewing skills, and eye contact, active listening, completing IEPs, assessing students, grant writing, maintaining anecdotal records, and giving back to the community. The volunteer experience was a platform for participants to develop as leaders in the community, build character, and become familiar with the culture of the community. Additionally, this experience introduced all of the participants as new community member or professionals in the community.

\section{Life Experiences}

The participants believed they had professors who provided them with knowledge that they "could not experience from a textbook" and could only experience first-hand in actual classrooms. Experiences included personality conflicts, hungry students, students with hygiene needs, or student without school supplies. The experience was a rewarding experience because it provided them with authentic classroom life experiences. Sharon described her first classroom experience with students who did not have the basic school supplies necessary for their success as students; this classroom experience taught her how to identify and address the needs of her classroom. She said, "There's no book to prepare

you for the students' life lessons, when you get inside the classroom, and the spontaneity of the situations, particularly in the inner city, in different neighborhoods." She indicated 
that teaching in the inner city taught her a life lesson and that she utilized that lesson to make her curriculum work. The daily classroom activities and challenges cannot be taught in teacher preparation programs. This was an authentic real life experience that Sharon did not believe would be present in the classroom. What she observed were behaviors and needs that she did not expect to experience in the classroom. Sharon indicated that the experience had deep meaning for her because she was able to provide basic necessities (paper, pens, pencils, erasers, and lotion) for a number of students in her classroom. Candidates are not instructed how to handle the abused or neglected student, the hungry student, or the student who does not have school supplies. The participants learned to follow their instincts to assist the students in the program without embarrassing them. It was a "learn-as-you-go" process. The participants indicated that, as field-based participants, they were introduced to the "school counselor," "how to complete individual education plans [IEP's]" for their students and the "school case manager." During the first week of professional development in the assigned schools, all of the candidates participated in classroom management training sessions. However, all of the participants expressed that "no one could prepare teachers for the needs of the individual students in their classroom."

Lucretia indicated that the participants were paired and assigned to several different elementary schools in low socioeconomic areas. She said, "It gave me a real life experience as a teacher and what teaching would be, instead of in schools that were cookie cutter schools where everything was perfect and it helped me interact with inner 
city students." She appreciated being placed in a school where she could experience diverse issues students and perform the daily duties of a certified teacher.

Latonya did not give the impression that she had the same overall experiences as her peers in the program. She indicated her experience was enlightening because it allowed her to realize that she enjoyed talking with the students and helping people work through their problems, but she did not want to be a classroom teacher. Latonya said, "This experience assisted me in identifying that instead of teaching in a class with 30 students, I'd rather be sitting in the office with one or two at a time. I feel that I could do much more and would be a great success in that area with me."

Although there were a number of similarities among the participants' experiences, there were notable differences within those experiences. For the first 2 years of the field-based teacher preparation program, volunteer work was not part of the curriculum. There were notable differences in the experiences with the school mentors and university mentors. Other differences included support, guidance, availability, and resourcefulness. Additionally, the participants indicated that the professor and supervising teacher experiences varied.

\section{Preparation}

Preparation was the third theme that developed. Preparation related to preparing the participants for the teaching profession and developing their understanding of the teaching profession. Significant details developed that supported the theme of preparation: one-on-one mentoring, teacher efficacy, as a first year teacher, and understanding the role as an educator. 
Preparation included a wide range of knowledge and skills that developed participants for the teaching profession. Participants brought an array of prior work experience to the program, such as working with youth programs, working in the science field, and working with community organizations. Sharon indicated that she had prior experience working with youth and teens in a variety of youth sports; Lucretia said," I was able to use the content knowledge for growth, and my pedagogical content knowledge was sound because I had a great science background and worked in the field of science." Yolanda indicated that she had experience working with at-risk youth in low socioeconomic communities in various programs. Sharon indicated that she was already working in the capacity of an educator. Although some of the participants had prior content knowledge, they gained critical pedagogical knowledge while participating in the program.

All of the participants indicated that their overall experiences in the program prepared them for the teaching profession. In relation to the theme of preparation, the participants made very strong connections to the overall experience as a significant factor in their preparation through the program as well as the ability to function in their future classrooms. Lucretia said, "The good thing about being in the program was I was able to use the content knowledge for growth." She indicated that the field-based program taught her how to teach. The program provided field-based participants with authentic and practical classroom experiences. Participants for this research were placed in different content area classrooms in elementary schools, middle schools, and high schools in the inner city. This allowed participants to gain real authentic hands-on classroom teaching 
experience. Clearly, field experience is a crucial component of the curriculum and program as a participant in a teacher education program.

Although each participant's experience was uniquely different, data analysis revealed that the participants were prepared far beyond the classroom knowledge they would gain as a traditional student in the university classroom. Participation in the program provided them with authentic hands-on experiences dealing with real life situations in the classroom, situations with culturally diverse students, the nuances of the classroom, and assessment strategies and communication skills. Understanding the dynamics of social interactions was significant for Sharon. Sharon shared that various issues would arise in the classroom, during volunteering, and when she would consult the program coordinator for feedback while participating in the program. She was impressed because the coordinator did not have a 'script' or 'book' in which to refer when she made recommendations to the participants. She described what she called a 'basic code' that was communicated and encouraged by the coordinator, "Be respectful, introduce yourself, and give them your sign in sheet." Sharon indicated one comment that resonated with her during the program, "DEAL WITH IT!"

Meeting the students was very crucial for Yolanda. She explained that she learned how to integrate the school-wide curriculum in her lessons to address the needs of the students and she learned how to prepare herself as a first year teacher. The program "gave me the opportunity to gain insight into the role of educators and learn how to get the students to buy into the curriculum regardless of their academic levels and all the baggage that they bring to the classroom." Sharon explained how the program developed 
her skills to address the diverse learning needs of the classroom and how to modify the curriculum materials based on each individual student in the classroom. She said, "The field-based training program allowed me to understand the whole issue of differentiation and that differentiation is not something that always comes with curriculum."

Clearly, this is not something that is taught during the methods courses of teacher training. This is a critical component for teachers to learn while in the field to assist in their development of culturally diverse classroom situations. Diversity is a major identifier as to how the demographics (gender, racial, and income) are changing in today's classrooms.

Preparing participants for the rapidly growing culturally diverse classrooms and behaviors that they will experience in the inner schools is critical to the professional development of first year teachers. The term "inner city" implies its location in a city or densely populated community that serves predominantly low socioeconomic income families and minority students. Preparing teachers to be successful in the inner city schools involves a field experience designed to develop the knowledge, skills, and way of thinking necessary to become highly quality and effective teachers immersed in the inner city schools.

The program focused on the participants' competencies, which developed the curriculum to address the diverse needs of the students, how to use the curriculum, how to demonstrate mastery of the subject being taught, the ability to self-reflect and set learning goals, and evaluate the success of the students. As Lucretia thought about her experience and how she became empowered while in the program, it reflected the 
curriculum and goals of the field-based program. She was able to understand various components of the program and apply various concepts in the classroom. The participants identified specific instances or situations that helped in their preparation. Both Sharon and Yolanda indicated that the program prepared them as professionals by conducting interviews, selecting professional attire, requesting letters of recommendation and refining their communication skills. Lucretia indicated the program prepared her by placing her in schools that were in low socioeconomic areas. Sharon indicated the fieldbased experience prepared her for the teaching profession. "It let me know, if I was going to teach, it showed me what I was up against with these kids up to that age and disrespectful." Both participants indicated that the experience exposed them to "homeless students", "students who didn't have parents", "students and parents who had drug issues, and students who were placed in inclusion programs." She felt overwhelmed by the experience with older children and realized that she would have to work with the same type of students in her classroom.

All of the participants indicated that, while participating in the program, they learned how to align instruction with the state standards to guide instruction and use a wide range of assessment strategies as a tool to evaluate student performance and differentiate instruction. Specifically, participants indicated that the field-based program focused on developing their ability to develop different forms of assessments, and develop student portfolios and journals. 


\section{One-on-One Mentoring}

During the interview process, it was clear that the one-on-one mentoring received by the participants from the coordinator and university mentors was a critical component of the program. It provided someone to confide in, model acceptable behaviors, and someone to assist in coping with societal issues in the classroom. Sharon indicated that the one-on-one mentoring from the coordinator and focusing on the nuances of the classroom were important in preparing her as a first year teacher.

Sharon shared some of the experiences that prepared the participants for the teaching profession that focused on the classroom. "Besides getting the one-on-one attention from the mentor, I was able to get real hands-on experience to be like a real teacher and actually do the things that teachers do on a day-to-day basis and not be scared to do it." She indicated that her self-confidence for the classroom grew as a result and that she was able to monitor the classroom, perform on-going assessment, transition between the lessons, develop a number of skills that taught her pacing, and identify and address the needs of the students.

\section{As a First Year Teacher}

All of the participants believed that, after completion of their program, they were ready for their own classrooms as first year teachers. They were prepared to handle the diverse situations, and they were ready to take control of their own classroom. The hands-on experiences provided to each of the participants while in the program exposed them to professional skills that would allow them to secure a job as a first year teacher. Experiences of the participants included effective communication, interviewing, lesson 
planning, classroom management, assessment, collaborative teaming, relationship building, and volunteering in the community. Participants also learned how to align their lessons to the standards, how to use a range of assessment strategies to evaluate student performance, and how to differentiate instruction. Specifically, participants indicated that the program focused on formative and summative assessments and the use of portfolios and journals. Not only did the experience prepare teachers for their first year in the classroom, it also prepared them for the work force. Participants gained critical skills that would be applicable in any career field.

All four of the participants agreed that the experience prepared them for the teaching profession as a first year teacher. For example, Sharon clearly communicated confidence as one of the critical skills that prepared her as a first year teacher.

Yes, my confidence was there. YES! I knew I was ready as far as confidence and being physically prepared. So, yes definitely my confidence because once you got the confidence, you act like you been there before and then you're good to go.

Lucretia agreed that the experience definitely prepared her as a first year teacher.

Participant ratings at the end of the program were what Lucretia used to validate her preparedness as a first year teacher. She indicated that all of the field-based participants in her program received superior ratings by their supervisors and were hired after completion of their program and student teaching. She agreed, "Yes, I was prepared." All of the participants indicated that the observations and semester ratings were used as an assessment tool to rate them in the areas of knowledge, skills, delivery of instruction, effective communication, and to determine if participants qualified for student teaching during the next semester. LaTonya viewed her own preparedness as having the ability to 
walk in the classroom with strong classroom management skills. She said, "Yeah, I can go into a classroom because it is in me." Although LaTonya felt that she was prepared as a first year teacher, she determined that she could not tolerate the students being disrespectful, thus deciding to remain in the teaching profession but in another leadership/administrative role.

\section{Understanding their Role as an Educator}

The role of an educator is very complex. It involves understanding the dynamics of the classroom, flexibility, understanding diversity, planning, team building, and professional development. With these participants, understanding the role of the educator included assessment, mentoring, and modeling, giving back to the community, alignment, development, and reflection. Two of four of the participants identified mentoring and modeling as necessary in understanding the role of an educator.

Sharon understood the role of an educator as mentoring students, new teachers, and colleagues, as well as providing direction. This enabled the participants to develop as professionals and understand the culture of the school. She explained that during her 2 years of teaching in the field-based program, she developed an important skill called mentoring. She said, "I am very quick to take someone under my wing and say, let me show you something." She stressed the value of having a mentor to go to for clarity, support, or just to have someone to engage in conversation was like a "security blanket." She indicated that having a mentor was necessary for her and it was an "invaluable experience." She believed that having a mentor is invaluable and that she was able to build from that experience as a first year teacher. 
Modeling for the students was a critical component for LaTonya. She stated, "I understand that I have to be a model figure for the kids." She understood that her role was not to become friends with the students, but to teach the students to become active listeners and model this for them. Sharon believed that modeling active listening skills opened the lines of communications with the students, and the student began to develop a level of trust with the teacher.

Giving back to the community is how Lucretia understood the role of an educator. She explained that her role was to "not only give to the community of students who already has everything but she feels better teaching in a low income neighborhoods." She believed the best way for her to give back to the community was to identify the lowincome neighborhoods that would provide her with 'real' authentic teaching experiences in a "real world setting." Sharon thought giving back to community was critical for all of the participants because it allowed the each of them to develop students from low-income communities or who experience academic difficulties, develop their self-confidence, and understand that being a product of the community does not constitute being a failure.

Reflection was critical in understanding the role of an educator for Yolanda. Reflection allowed the participants to assess their daily practices, identify room for improvement, and recognize their individual strengths and weaknesses as an educator. As an educator she understood that she must be "very careful and honest with the students" and that her role allowed students to "connect' with her because what she did and said "will have a lasting effects on the students." She communicated that the program taught her the importance of connecting with the students. She stated that there was a "need for 
her to be able to connect somewhere with the other participants, and they were connected like a family." Yolanda explained, "The field-based training program says that this particular time and space we are a family, we are a community, and anything we say here, we exchange between each other and we have an opportunity to share."

The field-based program provided several opportunities for teachers to develop as first year teachers. Teacher preparation training went far beyond the classroom and student teaching. It required participants to absorb knowledge through collaboration with other participants, professors, and mentor teachers, to volunteer in the community, and to self-reflect on their classroom practices. All of the participants indicated that the fieldbased programs helped them understand what it meant to be a teacher and what it took to be prepared for the first year in the classroom.

\section{Teacher Efficacy}

"Teacher efficacy" was the fourth theme that developed during the data analysis process. Specific details that enhanced teacher efficacy included collaboration, mentor teachers self-reflection, feedback, and developing classroom management skills. Teacher efficacy is defined as self-confidence and self-actualization that is based on the ability to connect with a diverse population of students and assist in their academic development. In this study, teacher efficacy differed for each of the participants. The prior experience and social constructs brought to the program also influenced teacher efficacy. According to the participants, many factors contributed to their self-efficacy as a teacher. These factors include improved pedagogical knowledge, weekly reflection, classroom management, and collaboration. 


\section{Collaboration}

One element developed during the data collection process was collaboration. All of the participants indicated that collaboration was a critical component of teacher preparation because it fostered team building, reflecting on their classroom pedagogy, their ability to connect with the students and promoting academic success in their classrooms. Sharon said, "Collaboration is the key. New teachers needed mentors to describe "the expectations of the administration or how things are done at that particular school." All of the participants indicated that their confidence increased significantly in relation to their ability to teach, classroom management, and meeting the needs of their students. Participants also indicated that they were able to meet the challenges of the classrooms in low socioeconomic schools.

Having a program mentor or school-based mentor played a critical role in teaching the participants how to navigate within the social constructs of the school culture, develop strategies necessary to collaborate as team members, and develop selfefficacy. All of the participants agreed that their self-confidence evolved as they participated in the program and that they developed teaching skills and classroom management strategies, which are necessary as classroom teachers. Sharon said, "I went from the internship to student teaching and was able to have nonchalant attitude." Lucretia said, "I was a potential, certified teacher with a great deal of confidence. I was offered a job while completing student teaching." Yolanda said "I developed the skills and knowledge necessary, and wasn't nervous about taking over the class." Sharon said, "I was ready and I had confidence and efficacy; I felt good about the teaching field." 
Conversely, one participant was unclear if being a participant in the program enhanced her own teacher efficacy. Yolanda said, "I don't really know if it, the teacher relationship within the field-based program, enhanced teacher efficacy." She indicated that the program taught her how to be sensitive to the staff and students in her communication efforts, how to "choose her battles" and develop effective communication skills with other teachers in the school and program and how to share information. Sharon realized that many of the participants had different academic majors, and that she had to learn not to be "judgmental." She said, "The program humbled me. I had to learn to not discern myself with the participant's reasons and respect them." She realized that the program taught her to "become a better person."

LaTonya indicated that the program enhanced trusting relationships between the participants, the mentor teachers, and university professors. This was evident when LaTonya said, "one of my supervising teacher's divulged critical information pertaining to how the schools receive money per student, and personal information about the students." This information was based on whether or not students were identified for student services, specifically, students with special needs IEPs. This showed that the teacher mentor respected the field-based candidate, and the participant could then differentiate instruction.

In their efforts to develop self-efficacy, participants engaged in weekly selfreflection, self-actualization, and peer feedback. Valuable time was given for participants to engage in constructive feedback with their peers, discuss classroom and community challenges, share ideas and strategies learned, and develop effective communication 
skills. This level of comprehension and engagement developed the participants' abilities to identify and connect with the school community and to share their field-based experiences with their peers.

\section{Disposition}

Knowledge, skills and attitude can be grouped together to define the participants' dispositions in the field-based teacher preparation program. The researcher analyzed how the daily social interactions among the field-based participants produced specific experiences in the program and which experiences could validate their meaning of the knowledge that constitutes the field-based program. The program taught problem-solving skills as the participants transferred classroom learning from the educational theory and were able to apply the knowledge to real-life situations. Each participant explained how he or she took control of his or her learning by actively engaging in the teaching and learning process. For example, participants explained that when they entered the program, they lacked specific knowledge and skills that were necessary as a teacher. After participating in the program, they developed confidence and grew as an educator, and they learned how to deliver instruction. The program allowed them to reflect on skills that they needed to develop personally and professionally, and some of the participants believed that the program needed to offer more workshops on lesson planning, basic skills, and teacher certification requirements.

\section{Knowledge and Skills}

Another sub-theme that stood out with all of the participants was the knowledge and skills gained as participants in the program. Participants found that the program 
enabled them to gain a clear understanding of the nuances of being a teacher and gain knowledge about the school community and culture. Skills included gaining experience with the district's grade book (in-putting grades, taking attendance, and communication with parents, students, and administration), delivery of instruction, and classroom management. Participants also indicated that it was very beneficial working in a real school and experiencing various diverse situations in the classroom that could not be taught from a book. They also indicated that their knowledge and skills as educators improved through the placements in different schools and communities and having a wide array of university professors and mentor teachers.

LaTonya indicated that the program taught her knowledge and skills related to the paperwork required by the field-based school principal. She said, "I learned how to create integrated lesson plans, maintain anecdotal records, use different teaching strategies and assessments, and how to work with IEPs for any students." LaTonya indicated that the knowledge that she gained from working in the schools helped her to realize that she wanted to work in the classroom. She was able to see the challenges of being a classroom teacher. She also indicated that the program assisted in her ability to self-reflect and recognize that she needed additional work on her writing skills. "Well, the good thing about it, I was able to use the content knowledge and pedagogical content knowledge for growth, and being in the program taught me how to teach." She said, "It came to me that I was living in a bubble as far as being a teacher was concerned. It is not a glamorous life." All of the participants agreed that having access to the university professors and mentors assisted in the development of their classroom pedagogy to improve their 
teaching skills because they were able to engage in dialogue with the professors and mentors regarding their delivery of instruction. Yolanda indicated that something very important to her was the feedback that she received from the mentors and professors. She responded, "I valued the weekly reflection once a week because we were given feedback from the professors, mentor teachers and other field-based participants." The participants learned how to receive positive constructive criticism from the program mentors and professors to improve classroom pedagogy. Weekly reflection was important to her in understanding how she could give back to the program, "like doing this interview and receiving feedback from the mentors and professors." It was important to her to identify how she could give back to the program or program participants. Yolanda indicated that, outside of volunteering, she was not giving back to the program. She stated,

Doing this interview was a way for me to give back to the program. I believe that it is important that I can give feedback about my learning and the strengths and weakness of the program to offer suggestions for the future.

The program also taught the participants how to ask questions and utilize available resources to gather information. Yolanda indicated that the coordinator had a specific skill that stood out during the program, "If there is something that the coordinator doesn't know or have, or she can't give you, she will say, 'I don't know let me find out'." She believed that learning how to ask important questions and being able to get the necessary resources provided her with an opportunity of emerge as a quality teacher.

\section{Collaborative Relationships}

The sixth theme that developed from the transcripts was "relationships." Developing relationships is an important skill in all career fields and is critical to the 
culture and community of the school. The participants' thoughts on the collaborative relationships were analyzed to identify the social implications as they related to the program. The socialization process developed a sense of community and a collaborative working relationship between coordinator mentors, participants, and professors. The significant details that emerged under the theme included professional relationships, coordinator relationship, trusting relationship, and participants' strengths and weaknesses.

Relationships were identified as significant details that played a vital role in the developmental and learning process that contributed to the preparation and success of the participant while in the program. The social constructs of the field-based program encouraged relationship building, team building, collaboration, culture in the work place and a sense of being. The field-based program served as a cultural foundation for the participants, where they were able to learn how to work and communicate together in a socially acceptable and professional way through building relationships.

In regard to the collaborative relationships in the program, the researcher analyzed how the nature of the program brought meaning to the participants through their daily social interactions. The daily social interactions were influenced by the participants' prior knowledge that was brought to the program, the on-going learning process, and interactions with the program participants, mentor teachers, and professors. During their participation in the program, the participants developed and associated their own personal and interpersonal meaning as they interacted with the principal and school staff in their assigned schools. Sharon described a relationship that she developed with one of her 
students as "priceless" because it was rewarding, and she unexpectedly made a connection with many of her students. One particular student that stood out for Sharon was very quiet and withdrawn but, as the year progressed, Sharon saw substantial growth in the student. She said, "I saw her first smile in the classroom." The student and teacher developed a strong bond and trusting relationship.

All of the participants' dialogues concerning program relationships indicated a level of cohesiveness within the program; conversely, some of the dialogue described negative relationships due to the actions of the school principal, supervising teachers, and university professors. Lucretia shared her experience with the principal and teachers who modeled to her an unacceptable social behavior toward the participants as the school leader. Lucretia indicated that her experience with the principal and teaching staff at her first field-based school was negative and were very disrespectful, and they exhibited unprofessional behaviors toward the field-based participants. Lucretia said, "The principal did not model a positive or accepting behavior nor did she make me feel welcome in the school." Lucretia explained, "The principal and the staff would not speak to the field-based participants and had a superiority complex about them." This experience allowed Lucretia to realize that she did not want to develop the aforementioned behavior. Lucretia said. "Well, I know when I become a teacher, I will not become like that." This behavior was perceived as socially unacceptable because school leaders set the tone of the school, and the principal can heavily influence the school culture. The staff and students emulated the behavior of the principal. If the principal displayed negative behaviors, then the culture of the school became negative. 
Relationships were associated with team building, which prepared teachers for the workforce and opened the lines of communication between supervisors and employees.

On the other hand, Lucretia said she believed that, as one of the original participants in the program, her interactions and relationships developed a foundation for future participants and recommendations for future implementation. LaTonya discussed the importance of the relationship that developed with the mentors, professors, and community that assisted in her development as a first year teacher. She explained that it was important to build the relationships because "you may need that person later in life." She shared how developing relationships helped with networking, developing social and communication skills, and assisted in developing strategies to problem solve.

\section{Professional Relationships}

Critical to this research were the professional relationships that developed among the professors, school community, program mentors, and participants starting the first day in the program. According to the participants, the professional relationships developed as a result of participating in the program and they fostered collegiality that remained after the commencement of the program. This was clear when the participants indicated that the program laid the foundation for them to return to the program to secure references for employment, seek assistance with employment, and academic advising. In communicating her field-based experience, Sharon was euphoric as she described the relationships that she gained in the program. She discussed how she began volunteering in the community and participating in the schools and the university. She said, "I began volunteering during the first picnic with the College of Education. I had to build the 
foundation that I wanted to be around and whom I wanted around me." She believed that by doing this, she was able to develop strong professional relationships where she felt comfortable returning to the supervisors to request letters of recommendation for future employment and scholarships.

LaTonya described another example of developing a professional relationship with the teacher, mentors, and professors in the program. She indicated that the coordinator provided them with specific rules of conduct while in the program. Those rules were when you go somewhere, introduce yourself, shake their hand and say, "Hello, how are you? My name is, while looking them in the eyes." LaTonya explained that this helped her develop trust with her superiors and future employers, built friendships and a positive working relationship. She said that she learned the importance of "meeting people and introducing herself."

\section{Coordinator}

All of the participants agreed that support from the field-based coordinator and mentor was very critical to the success of their development as professional educators and to the program. Yolanda said that having the coordinator as her mentor was a very rewarding experience and she valued their relationship. She explained, "The coordinator served as her mentor and taught her professional skills and methods of communicating." Yolanda stated that she wanted the researcher to understand that she was participating in the research study because of the relationship developed with the coordinator and that she "believed in the coordinator." LaTonya explained, "Without that experience with coordinator, without her being my mentor, I would not be as open as I am with the other 
teachers." She learned how to be receptive to the feedback from the professors and other participants during reflection and when asking for feedback for improvement.

Additionally, LaTonya indicated that having the coordinator as her mentor helped her learn some valuable skills, such as strategies in handling specific situations, refining her dialogue, and effective communication skills. She explained, "I think working with the mentor in the field-based training program taught me tact and perseverance. I trust what she did for me was so beneficial that I would do anything for her." This relationship indicated that the coordinator saw potential with these participants. Trusting relationships build character in the workplace and build a positive school culture. All of the participants indicated that the coordinator worked collaboratively with the participants in the program and stressed the importance of asking questions to clarify and maintain professionalism at all times.

\section{Trusting Relationships}

Another significant detail that developed under the theme of relationships was trusting relationships within the program. During their social interaction in the program, all of the participants indicated that they developed very trusting relationships with each other and the professors. According to the participants, the relationships with some of the professors, coordinator, and mentors fostered strong and supportive relationships. This was evident with the participants' comments about the trusting relationships. Sharon and Yolanda indicated that the program was more like a family; the participants trusted each other and the relationships continued after the completion of the program. Lucretia indicated that the program helped her develop relationships with everyone in the 
program. She indicated that those relationships were very important to her because she valued the comments and suggestions of everyone. She said, "The relationships helped me develop as a teacher while in the program." She indicated that relationship helped her open up to the other participants in the program, and that she knew that she could always talk with anyone in the program, "ask for advice" or "provide feedback." LaTonya indicated that she developed a positive working relationship with her mentor teachers. She discussed how the supervising teacher would model a typical day in the classroom, including classroom management skills and how to complete the mandatory anecdotal and daily records. She explained how the level of trust developed with her mentor was visible, "because he turned the class over to her after observing him for 4 weeks. Later, the principal gave her an additional class. She said, "The mentor teacher turned the class over to me, demonstrated what to do, and he trusted me with his class and the other teachers." She felt that her mentor developed a level of confidence in her ability to apply her teaching skills in the classroom.

\section{Participants' Strengths and Weaknesses.}

Another benefit of being in the program included the professors' abilities to identify the strengths and weaknesses of each of the field-based participants. All of the participants indicated that working with the mentor teachers allowed them to develop as a professional and prepare as a first year teacher. Yolanda shared how she had a problem with controlling her temper and speaking loud or in an abrasive tone. She explained how the coordinator used specific language to provide and receive positive corrective feedback to correct and develop her tone of voice when speaking to people. She said, "the 
coordinator would tell me, always, think about what you want to say, take a step back before you respond; if it doesn't affect you or your classroom directly, then do not entertain the issue." Working with the coordinator who also served as her mentor taught her to speak with an acceptable tone and develop a language that was conducive to the educational environment.

LaTonya shared that the coordinator knew that she had difficulty with assessments and generating lesson plans. The coordinator arranged lesson plan workshops and assessment workshops for all of the participants without identifying her. The program curriculum included generating lesson plans and reviewing them with the program mentors, colleagues, and classroom mentors and assessment preparation. All of the participants indicated that they had to generate weekly lesson plans and collaborate with the mentor teacher to assure that the lessons aligned to the school curriculum and were integrated across the curriculum.

Developing relationships among the participants was critical in their social development as teachers. According to the participants, relationship building played a major role in the participants' learning during their field experience relationships. It built a sense of commonality, promoted team building, and developed teacher self-efficacy. The aforementioned significant details are examples of the participants' feelings of the factors that contributed to their negative or positive field-based experience. All of the participants agreed that the field-based experience with their peers, the various schools, and university mentors was critical in their development. Significant factors influenced the value of relationships within their experiences, which they identified as "family." All 
of the participants indicated that they developed a sense of belonging as a result of the relationships developed in the program. Although there were some notable negative fieldbased experiences discussed, these experiences did not alter the participants' learning.

\section{Program.}

The seventh theme that developed was the field-based program. The definition of field-based program is the nuances of the program curriculum, the professors, the participating schools, participants, and the coordinator. A number of strengths and weaknesses were identified among the participants of the program. The strengths and weaknesses discussed included leadership, financial resources, prepared participants, and participating schools. The weaknesses that emerged include lack of participating schools and lack of university support.

\section{Strengths}

The researcher analyzed the perceptions of participants in this program to identify the strengths and weaknesses of the program and the experiences the participants found beneficial to their development. When the participants were asked to discuss the strengths of the field-based program, they all acknowledged the value of the authentic classroom experience and their development and understanding of the school community. All of the participants indicated that, in addition to having strong support from their assigned mentor and program coordinator, leadership was a critical component of the field-based program. Latonya stated, "The program provided me with the opportunity to see what a real certified teacher experiences in the classroom daily." Sharon indicated that the program provided her with opportunities to see great leadership by observing the 
program coordinator and professors. All the participants indicated that the program provided them a strong team of university professors and classroom teachers who were concerned with their development as educators. Sharon said, "YES! I think the strengths were definitely that the program coordinator and professors provided leadership." Participants had opportunities to lead in their classrooms and other community based activities. These activities included volunteering at the school picnic and at the Children's Museum. All of the participants indicated that the program assisted in their development as leaders because they were able to take charge of their assigned classes. Additionally, the participants experienced different leadership styles among the supervising teachers and the university professors. Last, all of the participants indicated that, after completing the field-based program, they developed self-confidence, knowledge, and experience necessary for their success as educators.

\section{Financial resources}

Another important factor that benefited the students was that the program provided tuition scholarships to participants who applied and qualified. They indicated that finance was an issue because they had to travel to the different sites, to the inner city university, and to volunteer in the community. All of the participants indicated that they had to report, remain at the assigned school all day, and were not able to work while participating in the program. A tuition scholarship and stipend for classroom materials was a welcome relief to their strained financial situations. Sharon explained: "Having a stipend. I mean, I'm glad that somebody gave us money. So, I think that was definitely a benefit." Yolanda indicated that she had to work, which limited her participation in some 
of the professional development activities. All of the participants indicated that the financial support from the program assisted them in purchasing classroom supplies to assist with their instruction. Additionally, if the financial resources were not available, the coordinator provided the participants with the needed classroom materials or supplies. Lucretia stated, "If the coordinator knew that the participant needed something, and she had it, she would tell us to walk with her to her car. She would give us materials from the trunk of her car."

\section{Prepared participants}

Participants were able to compare their knowledge and skills with other participants in the program and believed that they were capable of handling the demands of a self-contained classroom. Lucretia viewed one of the strengths as the ability to prepare the participant as first year teachers. She believed many programs during that time only provided student teaching as a means of real world, hands-on, authentic experiences prior to becoming a certified teacher. She said, "Well, the strengths of the program were that it prepared all of the participants in the program to become teacher before you actually go into teaching."

All of the participants viewed mentoring support as a strength of the program. Having someone who was as dedicated about their development as teachers and having someone who worked hard to ensure that they had what they needed assisted in their growth as a professional educator. This was crucial to the participants in the program because it exposed them to another level of classroom experience as teachers. They had exposure to opportunities where the coordinator and teachers wrote and applied for 
different grants to purchase classroom supplies and materials. Yolanda explained why she viewed this as one of the program's strengths. "So, it is about having someone who goes out of their way, who has a passion about this program as I do being a teacher in my classroom." As a result, the coordinator and participants had to network with other university staff and community members to gather resources such as materials necessary for the classroom lesson. Another important strength of the program for all of the participants was the placement in an actual classroom working hands-on in the classroom in lieu of just observing. All of the participants indicated having that opportunity was of high value to them.

\section{Participating schools}

Originally, there were a limited number of participating schools available for the participants because many school administrators were unaware of the program. However, Lucretia indicated that she was placed in two schools that were on academic probation, a specialty arts school, and an achievement academy. Yolanda said that she was placed in three schools on probation and in schools that had a student exchange program, school banks run by the students, an import and export program with Africa, and school gardens maintained by the students. As the program grew, the availability of schools for the participants to gain hands-on experience increased. This was one of the strengths outlined by three of the participants. Lucretia shared what she viewed as strength of the program. "OK, I found the strengths of the program were them placing us with the various schools, getting us assigned to schools and communicating back and forth." She stated that the 
individuals who she reported to in the school were "very good, very strong and very important" to her while in the program.

\section{Weaknesses}

The lack of participating schools and the lack of university support were two weaknesses of the field-based program.

\section{Lack of participating schools}

During the initial stages of the program development, a limited number of schools were available to house the field-based program and participants. Sharon indicated, "The weaknesses of the program were that we did not have enough schools, and we did not have enough students who could benefit from this." She indicated that her first school placement fostered a great deal of controversy during the first semester, which promoted a negative school culture and climate for the field-based participants. She was surprised that the school administrator maintained a negative disposition with the participants in the program.

\section{Lack of university support}

Another weakness identified by Lucretia was the lack of support for the fieldbased program from the university. She felt that the field-based program was treated as a separate entity from the college of education and she had a difficult time with the staff when she needed information. She said, "When I would come in the education department, just to get a message conveyed to someone, I would ask the staff, "Why are you making it so difficult?" She viewed this as a weakness because she "experienced and 
observed discriminatory practices toward the program." In her opinion, the program functioned like a family, but the field-based program felt like a "stepchild."

Participants had a common experience in getting relevant information or getting resources from the university. The participants indicated that the university lacked support for the program by the lack of funding available to them and the coordinator not having the authority to make financial decisions that would support the participants in the program as it related to classroom supplies, stipends, and scholarships.

LaTonya indicated that there was a lack of organization. She said, "The weaknesses I found were that the program just lost the paper work." She shared how participants had to resubmit documents several times because the documents were constantly lost or the supervisors/instructors failed to submit documents to the college.

\section{Participant Recommendations}

The eighth and last theme that developed was "recommendations." Recommendations from the participants' perspectives were critical because they were based on their experience and what they believed was needed or missing from the program as it related to being prepared for the teaching profession. In the opinion of the participants, they each had their own recommendations that would enhance the fieldbased program, attract more students, recruit, and retain participants for the program as well as the teaching profession. In addition, their recommendations would improve participants' experiences, prepare candidates for certification, provide skills development and effective communications, develop collegiality between the university and assigned school mentors, and enhance university support for the program. 


\section{Financial}

All of the participants proposed that the program could offer more financial assistance or travel reimbursements to the participants in the program. Sharon said, "They could give a couple of more bucks more to the students because I think with you going around, you use your own gas, money getting around, people needed help all over." She indicated that lack of financial assistance was the main reason she remained close to the university in lieu of venturing out to other schools outside of the university community, because she was experiencing a financial strain.

\section{Expansion}

For approximately 20 years, the field-based program was housed in a neighborhood elementary school and participants were assigned to schools in communities with high percentages of low-income citizens. The program was limited to only those participating schools in the specific community of the university. Lucretia recommended that the university should consider ways to enhance the program by extending the program to other schools. She said, "To expand it to more schools, make it where practically everybody should be doing this not only a controlled group, because all of the students are going into the candidacy will have a clinical emergent program." She explained that the "clinical emergent plan" is the same as the field-based program that offers students the opportunity to experience the classroom prior to student teaching.

The field-based experience provided an opportunity for the participants to develop relationships with the students in high-poverty communities. Teachers in the field-based program did not experience teaching students who were from different racial or ethnic 
backgrounds that differed from their own. However, affording participants the opportunity to experience schools that are more affluent, high achieving schools, foreign language academies, or baccalaureate schools would provide a well-rounded learning experience for many of the participants.

\section{University Support}

Effective communication is critical to the success of any program. All of the participants indicated that there was a need to articulate and model consistency in communicating information regarding the field-based program and needs of the fieldbased participants. Although participants were placed in different academic programs, some indicated that required coursework from the professors lacked consistency. All of the participants indicated that the program appeared to be isolated or a separate entity from the university. There is a need for a greater sense of communication between the university and the field-based program, a high need to gain university administrative support, and a need to develop a level of camaraderie within the program and university to develop and maintain high academic standards and professionalism. Yolanda indicated that the program needed more support from the university, more professional development, more feedback, and longer field-experience. She said,

In order to support the program, it needed to be an additional person, or an additional portion of the program, that went out and just did open research on the different types of observational places that we can go, the different opportunities.

One of LaTonya' recommendations were to include a group of diverse mentors and provide mentoring training for them. She said, "Have a session set up for mentors on how 
they should be analyzing the relationship between mentors and the field-based candidates when they are assigned to the schools."

\section{Goals and Mission Statement}

This research revealed a need to develop a stronger goal and mission statement that clearly articulates the goals and mission of the program and describes how the program activities connect to the teaching profession. They must articulate clearly how the goals and mission correlate with the competencies required of the program. Participants had different perceptions of their roles as participants in the program. They interpreted the field-based program and participating goals differently in relation to the outcome of the program, community service, the procedures and the roles of the university and school mentors. Sharon said that she was one of the original participants of the program and that she appreciated how information was clearly articulated, and the goals and objectives were clear and discussed while in the field-based program. However, as the program coordinators changes, so did the curriculum, and how information was communicated. Lucretia, Yolanda, and LaTonya stated that the goals and objectives were not clearly stated and that they could not see how volunteering in the community was connected to the field-based program and their learning. Additionally, LaTonya stated that the goals and objects of the program were not written or given to them.

\section{Field-based Curriculum.}

Teacher preparation programs should design experiences that assist in the participant's comprehension of the philosophical, psychological, and social foundations 
of education as it applies to their content area. All of the participants discussed social issues that had a direct impact on the program participants. Participants indicated that there were some disconnections among some areas of the program. It was clear that the programs aligned to the state and national standards; however, unclear to three of the participants were the program goals, community connections, and how the program would assist the participants, as they would progress. A strong curriculum aligned to the state and Common Core Standards assures that the participants will have an understanding of the pedagogical skills, the content and instructional needs necessary for the teaching profession.

In addition, the curriculum should include a consistent level of mentoring. It appeared that the participants for this research had the same mentor. The field-based program should solicit professors from the university, participating schools, and administrators to serve in the role of field-based mentors to share the knowledge and skills necessary to enhance the academic success of the participants.

\section{Structured Community Service Program}

The participants recommended that a more structured community service program should be developed where participants clearly comprehend the purpose of performing community service and are able to connect the service to their learning and teaching profession. It is important to articulate the importance of community service and the role it plays in developing educators, self-efficacy, and leadership. LaTonya indicated that the participants were told that they had to perform community service in the museums, neighborhood, or on campus. She indicated that direction was not given to them on how 
to arrange the community service. However, near the end, she began to see the connection. She said, "We had to go on interviews, set up meetings with the directors of programs to perform community service, and we had to dress as if we were applying for a job." Lucretia said, "Performing community service developed my self-confidence in my ability to interview for a job, because I was not an outgoing person before I came to the program." Yolanda said, "For me, the program taught me how to communicate and prepare for interviews. We had to return to the companies where we volunteered and ask them for letters of recommendations for various scholarships.” Sharon said, I didn’t understand the role of volunteering in the community in the beginning but after volunteering in the Museums, I began to see the connection." Sharon also said that volunteering in the community provided an opportunity for her to learn about the various educational activities that were offered to educators for professional development and the various programs for students.

\section{Equitable Learning Experience}

Yolanda indicated that there was a "lack of equity" and rated the program on a scale of one out of three. She indicated that the coordinator gave the same to all of the participants and displayed equality on her part and was an equitable person. However, she rated the program with a three because she was disappointed that the coordinator did not have the authority to "run the whole program." She indicated that there were barriers that prevented the coordinator from providing for the program, such as limiting fieldbased activities, university support and resources, and university decisions without consulting the coordinator of the program. She indicated that the coordinator should be 
included on all decisions that directly affect the operation of the program and the participants in the program. The program should enable participants to develop their own philosophy, techniques, and approaches that are consistent with current theories and practices and that will foster equitable learning experiences. The program should provide opportunities for the participants to continue reflecting and analyzing their own practice and encourage continued positive corrective feedback from all university mentors and professors.

\section{Professional Development}

Yolanda stated that the program offered professional development to the participants but she believed that more professional development was needed. She also indicated that there was a time conflict because the participants were in the field during professional development hours at the university. LaTonya recommended that the program offer classes on assessment to prepare the participants for the certification exam. She said, "They need to offer a class on testing, and a class strictly focused on writing lesson plans, and really they need to drill the participants." Although LaTonya felt that she was prepared as a first year teacher, she indicated that she was deficient in writing lesson plans. She said, "They gave you a couple of exercises and had you write lesson plans, but it was not intense." Writing lesson plans involves more than a few exercises and showing the participants how lesson plans look. LaTonya wanted more instruction on the content of lesson plans and how she could differentiate her lesson plans.

Additionally, she indicated that participants needed state classes to assist participants on preparing for the state assessments and certifications. She said, "They need an intense 
class on lesson planning, and Assessment of Professional Teaching (APT) because it is a graduation requirement for all field-based participants.

\section{Feedback}

All of the participants agreed that feedback was a critical component of the program. Feedback was an opportunity to assist with the development of the program, an opportunity to provide recommendations for improvement, and an opportunity to selfreflect. Yolanda said, "I would definitely have more feedback, more reflection, and more feedback time." She indicated that some of the schools that they were assigned to were not receptive to the participant being placed there. She felt that many of the teachers and principals did not want the participants in their schools and did not trust the participants.

\section{Conclusion}

Kvale (1996) suggests that the aim in using interpretivism is to learn from the past in order to understand the present. The researcher attempted to understand the meaning of the graduates' experiences in the program to determine how the program helped prepare them for the present teaching profession. The participants' experiences in the program are essential to this research because the researcher is concerned with the participants' understanding of the experience and perception during the program and the authenticity of what they are sharing with the researcher. Their experience enhanced my understanding and brought awareness of the participants' experiences in the field-based teacher preparation program to better prepare teacher candidates, as well as assist in the development of future programs. In addition, my experience in collecting the data brought a certain level of sensitivity to the phenomenon because of the issues that 
developed during the interview. The reconstruction of the participant's field-based experience has significant implications for improvement in teacher preparation, student teaching, development of policy, and future field-based programs. It communicates the value each participant has for the program and the knowledge and skills that they developed as teacher candidates. 


\section{CHAPTER V \\ DISCUSSION, CONCLUSIONS, AND SUGGESTIONS}

FOR FUTURE RESEARCH

Chapter V presents an overview of the research, the purpose of the study, summary of methodological procedure, and recommendations for future research.

Conclusions based on the findings are discussed, and implications for field-based teacher preparation programs as it relates participants' experiences and perceptions and implications for future research conclude the chapter. The overarching research question is discussed based on the study findings.

The purpose of this research was to analyze the experiences and perceptions of participants from an inner city field-based teacher preparation program (INC/FBTPP). The objective was to provide a discussion on the data that answered the research question that guided this study and discuss implications future research. The question sought to analyze the experiences and perceptions of graduates from an inner city field-based teacher preparation program. Previous research suggested a need for improved fieldexperience that focused on programs, which connect both public school and university experiences (Holmes Group, 1986, 1990). Current research on field-based programs revealed that field-based programs curriculum have focused on programs that a committed to connecting to both the public schools and university experiences. It was 
clear that this INC/FBTPP has a strong connection with both the public school and university experiences. This research validates the argument that field-based programs have been identified as a critical means for redesign for teacher preparation (Metcalf, 2014; Darling-Hammond, 2014). It brings to mind questions and concerns that are discussed by participants in such program and questions if such program prepare participants for the teaching professions. Although the research provides a small sample, it confirms that the redesign in this field-based program provided the participants with experiences that prepared them for the teaching profession.

Traditional field-based programs offer participants clinical experiences during student teaching as a prerequisite of their teacher preparation program. This field-based teacher preparation program established a collaborative university and public school connection that compliments the inner city university teacher education program. As part of the IN/FBTPP, participants gained on-the-job training for 2 years.

This research investigated whether participants viewed themselves as well prepared for the teaching profession as a result of participating in an IN/FBTPP. Specifically, what are the perceptions and experiences of participants in an inner city field-based teacher preparation program in terms of perception of preparedness, knowledge, skills, and attitudes that are critical for success, and relationships and experiences with professors and mentor teachers? Participants' experiences and perceptions were critical components in understanding the culture, language and sociocritical constructs of the program and how such programs contribute to the teaching profession. The language, culture, and experience of each field-based school were 
different for each participant. Participants' experiences different leadership and modeling that was critical in their development. Having the opportunity to participant in the various professional development workshops, opening of the schools, and closing of the schools provided a wealth of information to the instructional and professional expectations of each assigned teacher. Participants gained hands-on experience in analyzing data, working in the community and learning how to effectively community with parents, students and staff. Furthermore, taking the first step to analyze the participants' experiences and perceptions provided rich authentic data for the future of field-based programs and began an exploration in an area that had yet to be investigated until now. The knowledge gained from the data revealed a wide range of responses to the research questions that attempted to answer the research question. Findings for the research developed throughout the interpretive exchange of meaning between the participants and the researcher.

\section{Theoretical Framework}

Interpretivism as a theoretical framework focused on sociocritical interpretation of participants' experiences based on their perceptions. Interpretivism fostered the implementation of phenomenology as a method of inquiry that enabled phenomenology, gathering, analyzing, and interpreting such data. Phenomenology as a research method furthered the tenants of interpretivism to determine the data collection method and analysis process, which was the foundation of this study. 


\section{Discussion}

The findings of this research add to the existing literature on field-based teacher preparation, specifically how field-based participants perceived their experiences, relationships developed, and preparation received in the program. The nature of the questions and the response from the participants resulted in the researcher using phenomenology as the methodology and combining some of the responses. Results of this research consisted of seven major findings related to the overarching questions. It was surprising to learning that each of the participants had a similar experience with the same field-based mentoring professor, however, their perception of the experience with the professor were different. I was surprised that the participants believed that the experience with the mentoring professor as well as the school principals taught them how to remain professional in the work place and focus on the language used in communicating with superiors.

Connecting the themes to the theoretical framework and its emphasis on understanding, the tenets of interpretivism develop the understanding of the "social world" or "being" language and culture, perception, experience, preparation, disposition, collaborative relationships, program, and recommendation. This prompted a review and analysis for implications of future field-based programs.

\section{Research Question One}

What are the perceptions and experiences of participants in an INC/FBTP in terms of perception of preparedness?

The data indicated that the participants' perceptions of being prepared for the teaching profession were very similar in terms of understanding how to generate lesson 
plans, classroom management, student assessment, delivery of instruction, and community engagement. It was disturbing to learn that none of the participants mentioned engagement with the parents. Learning how to communicate with the parents is crucial in the development of the students. Parents are play a critical role in the culture of the school community and the decision making process. However, all of the participants indicated that they were well prepared for the teaching profession after completion of the program. As reported in Chapter IV, 100\% of the participants reported that they were looking forward to successful student teaching and employment as a teacher at the completion of their program. As the participants reflected on their experience in the field-based program, they believed that the skills, training, and knowledge received in the program prepared them as future teachers. These findings were revealed that, although the field-based participants participated in the IN/FBTPP in different years, the program fostered a well-rounded holistic and rigorous experience. The participants suggested that they gained a wealth of experiences far better than what was required for their student teaching practicum and clinical hours. The field-based experience enhanced the participants' student teaching experience and provided a strong foundation for the participants of the program. Additionally, the research understood that the participants' perceptions of their experiences were heavily influenced not only by the coordinator but also by the language and culture of the program and the participant's social engagement with the other field-based participants and community.

Moreover, the data revealed that $50 \%$ of the field-based participants indicated that there were communication deficiencies between the university, field-based professors, 
and program participants. At the beginning of the program, it was clear that half of the participants did not clearly understand the goals and objectives of the program or understand how community service would connect to their training as an educator. All of the participants communicated differing perceptions of both the field-based teacher preparation program and the teaching profession. Three of the participants indicated that they were unaware of the level of commitment that was required of them. Further, two of the participants were aware that they would actually report to their assigned school as a classroom teacher. Two participants thought that they would report to the school and just observe the class, grade papers, and serve as a teacher assistant. One perception that stood out among all of the participants was that they thought they would continue reporting to the University for their Methods classes.

This brings to mine one question, what does this say to teacher education institutions as they seek ways to improve their current program or institutions that are creating a FBP? This research proposes that institutions should focus on effective communication in the delivery of information and recruitment for field-based programs. Current programs coordinators could utilize participants' reflections, recommendations, and feedback as a tool to guide or enhance the program. Additionally, assuring that the program goals and objectives are clearly communicated orally and in writing to enable participants to understand how the program is connected to the teaching profession and how the program will assist in their development as future educators. It reminds us that regardless of the level or age of the learner, everyone learns differently, communicates 
differently, and comprehends differently. As a result, it is important that we continue to differentiate instruction and work toward continued effective communication.

This research revealed that all four of the participants in the INC/FBTP believed that they were well prepared for the teaching profession as a result of having two years of field-experiences in different grade levels and school environments. Darling-Hammond (2008) proposes that affording longer field-experience allows the participant to capitalize on their experience and development as an educator, which will ultimately better prepare them for the teaching profession. All of the participants believed that the mentors, supervising teachers, and program coordinator provided them with the experience necessary to prepare them as professionals. Additionally, all of the participants stated that the relationships developed in the program were one of the strengths of the program. Creating a sense of community was critical to the participants' success. This was evident during the interviews, as all of the participants indicated that they looked forward to reflection sessions on Fridays because they had the other field-based participants to problem solve, provide scenarios, make recommendations, or just have someone to listen to them.

Additionally, the participants indicated that the program developed them as professionals by requiring weekly community service, teaching interviewing strategies, developing portfolios and resumes, and attending professional development workshops. Most importantly, all of the participants indicated that the program provided an avenue for them to develop social skills and long-lasting friendships with everyone involved with the program. All of the participants indicated that the program provided them with a 
strong foundation as first-year teachers and that the program was like an extended family for them. One particular development that elevated their personal and professional efficacy was the offer of future employment by the field-based school principals. All of the participants were offered employment after the completion of student teaching.

Moreover, the program altered the participants' perception of their roles as educators, community members, and their administrative responsibilities. Understanding how the field-based participants perceived the teaching profession was very important in developing them as future teachers. According to all of the participants, the teaching profession is a calling that requires them to receive students as there are in their classroom. All of the participants agreed that the teaching profession requires a deep understanding of the needs of the students and the wiliness to go far beyond the surface to secure resources for their classroom. Last, the participants indicated that the teaching profession requires a great deal of flexibility because it is a multifaceted profession and required more skills than what was previously perceived. Teachers were able to understand the connection between the field-based teacher preparation program and the teaching profession.

It was evident that the field-based program provided opportunities for the participants to experience diverse situations in the classrooms and gain professional development training in addition to their training as future professionals.

The participants were oblivious of the administrative responsibilities of being a classroom teacher and their responsibilities as a teacher in the school and community. The participants indicated that they thought that they were going to report to the school, 
create lesson plans, and then go home at the end of the day. Two of the participants' perceptions were that teachers had an easy job. As the participants progressed through the program, they began to gain a clearer understanding of the role of an educator and the qualifications necessary to gain employment as a teacher. At the completion of the program, the participants assumed that would not have to go through the application process with the school district because they were offered jobs by the principals. However, participants had to complete all of the mandated requirements by the state and school district, complete an on-line application, and submit the required district and state credentials necessary for employment.

After analyzing all of the data from the interviews, this research provided evidence that the inner city field-based teacher preparation program fostered positive collaborative relationships, and the program was in line with the current state and national standards for teaching and teacher preparation. Research indicates that teacher standards were developed for dispositions, new teachers, and knowledge and skills by organizations such as the Interstate New Teacher Assessment and Support Consortium (INTASC), National Council for Accreditation of Teacher Education (NCATE), and Teacher Education Accreditation Council (TEACH) (Rose \& Terndrup, 2007). All of the participants had high regard for the role of the coordinator, university mentors, and supervising teachers. Participants indicated that having a mentor was critical to their overall success as individuals and professionals. For all of them, having a mentor refined their communication and professional skills as future educators. This implies that the 
field-based teacher preparation programs with extended field experiences prepared the participants for the teaching profession.

\section{Research Question Two}

What are the perceptions and experiences of participants in an INC/FBTP in terms of knowledge, skills, and attitudes that are critical for success?

In this research, it was necessary to classify knowledge, skills, and attitudes as dispositions to demonstrate their connections to education. In his discussion on candidates' dispositions, Rose (2014) indicated that many organizations have developed standards that focus on teacher candidates, dispositions, knowledge, and skills. However, NCATE recommended that universities develop their own standards for the aforementioned. Additionally, Rose (2014) suggested that, in the educational arena, dispositions do not have a clear definition. He continued by stating that dispositions are the "habits of heart and mind" that is brought to the teaching and learning community by the teacher candidate.

After analyzing the interview data, the research concluded that knowledge, skills, and attitudes (dispositions) were very important to all of the participants in the program. All of the participants indicated that they developed a wealth of knowledge and skills that would prepare them for the teaching professions. This was evident when the participants indicated that the field-based teacher preparation program served as the foundation for their transition from teacher candidates and field-based interns to teaching professionals. All of the participants indicated that they possessed a high level of self-efficacy because of their experience in the various schools and the preparation as a teaching professional. However, one participant indicated that her field-based experience assisted in her 
realizing that being a self-contained teacher or a specialized teacher was not the profession that she wanted to explore. She indicated that she wanted to develop in another role as an educator.

The field-based teacher preparation program developed the participants' overall knowledge and skills necessary to perform the job duties as successful classroom teachers. These included such skills as weekly lesson plans, weekly assessments, and maintenance of anecdotal records. Participants acquired skills that prepared them to deal with adversities that they would encounter in the classroom, specifically, students at risk for failure and students needing behavior modification. The knowledge and skills that were of high value to the participants were the ability to connect with children and parents, facilitate peer workshops, implement Response to Intervention (RTI), and create and implement Individual Education Plans (IEP's) for students. Field-based participants learned the language of the school, and school practices and developed knowledge about the culture of the school. Additionally, the data revealed that the participants gained a clear understanding about the role of power and knowledge in the field-based program and the school system. McLaren (2007) explained, "Knowledge is something to be mastered and power is discourse in education or a regulated system of statements" (p. 209). Knowledge and power in the educational system dictates the books that guide the curriculum, classroom rules to employ, and the values and beliefs of the school. The participants learned that each school culture and rules were different and that they had to be receptive to the knowledge and power of each school. 
Participants developed a deeper understanding of how their own culture influenced or shaped their perceptions of the teaching profession, the field-based teacher preparation program, and their own learning. It is safe to say this experience validates that the field-based participants gained knowledge and skills that classify them as highly qualified teachers, according to this State Board of Education. This also validates that the field-based program adheres to the rigorous learning standards set by this state and outlined in the Common Core Standards (2014) for teacher preparation programs.

\section{Research Question Three}

What are the perceptions and experiences of participants in an INC/FBTP in terms of relationships and experiences with professors and mentor teachers?

After analyzing the data, it was evident that relationship building was a critical component of the field-based teacher preparation program. Research advises that building communities and relationships contribute to learning (Koeppen, Huey, \& Connor, 2000). From the perspective of the researcher, the field-based teacher preparation program modeled how to build relationships in the workplace and community while participating in the program. This was clearly communicated by the participants during the data collection process. All of the participants indicated that the field-based coordinator, university professors, and mentor teachers modeled the expected behaviors of the participants and encouraged developing professional relationships by everyone in the program. Recent research on teacher preparation proposes that community building fosters learning and discourages the intellectual and professional isolation of educators (Conner, 1996; DuFur \& Eaker, 1998; Goodlad, 1994; Koeppen et al., 2000; Tinto, 1997; Sergiovanni, 1994). 
Another component identified by the participants that enhanced the relationships with the field-based participants and professors was the weekly reflection and method courses at the assigned field-based elementary school. It was clear that the weekly reflection fostered what Yolanda termed a "Like a Family" atmosphere. The overall experience in the program, with mentor teachers, administration, the school community, peers, and university professors, was both negative and positive. All of the participants indicated that they perceived the experience as very critical to their personal development and that their field-based learning was enhanced by the relationships developed and the weekly reflection. The program provided a wealth of experiences for the participants. All of the participants' field-based experiences included community service, administrative work, staffing, Individual Education Plans (IEP), parent and community relations, and professional development. Specifically, the field-based experiences enabled the participants to manage their own classrooms. This is an indication that participants were prepared to address the needs of the classroom, public relations, and working collaboratively with their peers. Finally, the program developed the participants' understanding of how differentiated lessons addressed the needs of students across the curriculum.

In developing relationships, participants indicated that feedback was vital to their success in the program. Field-based participants developed positive collaborative relationships among their peers, professors, mentor teachers, university mentors, students, parents, and community members. Participants met weekly to engage in dialog with the university professors and coordinator, and the university professors visited the field- 
based participants weekly at their assigned schools. This suggests that relationships and community building in field-based teacher preparation programs played a critical role in developing teacher self-efficacy. Participants developed self-confidence in their ability to deliver instruction across the curriculum and address the needs of their students. They also indicated that having on-going collaboration with the mentor teacher, coordinator, and other field-based participants developed their social skills as professionals and taught them how to communicate effectively. Most importantly, the participants indicated that having on-going collaboration provided a venue for them to demonstrate problem solving with their peers, provide emotional support, and provide and receive positive constructive feedback during reflection, which encouraged each other in the program. This information is important because it provides recommendations for future teacher preparation programs based upon the participants' experiences.

\section{Personal Perspective}

As one of the first participants in the IN/FBTPP, my field-based curriculum included and additional component. I decided to apply to travel to Nakajo, Japan, for 7 months while in the program. This decision came with a lot of expectations and guidelines, and I was ready for the experience. My home university required that I adhere to the INC/FBTPP curricular guidelines and meet all deadlines, no exceptions to the rule. This included conducting research titled A Comparative Analysis of Education in Illinois to Japan and presenting my findings to the field-based professors and department chair upon my return. In addition, I had to adhere to the field-based teacher preparation program in Nakajomachi Kitakarabarakan, Japan, prefecture (prefecture is the first level 
of jurisdiction and administrative governing body in Japan, 日本, which consists of districts, cities, towns, and villages) to study the educational system in Japan and participate in the teacher preparation program.

The Japanese field-based teacher preparation program (JP/TPP) provided me with a holistic teaching experience on all educational levels (public schools, primary, lower secondary, upper secondary private, mountain, college, university, and special education facilities). The curriculum consisted of following the teacher preparation guidelines in Japan, which included elementary, middle, and high schools, mountain schools, universities and special education facilities. This experience taught me a great deal about working with diverse learners, students who were English-language learners, and students from other countries. More importantly, this experience taught me how to embrace the various cultures in the classroom necessary to educate the students, how to make the students feel part of the class, how to help other students become comfortable with the diversity in the classroom, and how to differentiate lessons. I had a language handicap because all of the schools were taught in the Japanese language, but the students (gakkusai) assisted in my ability and development in speaking and writing the Japanese language.

Immersion in the Japanese educational system developed my Japanese language skills, and Japanese teacher candidates worked on their English language skills with me. In Japan, I learned the schools had to adhere to the guidelines of the prefecture not that of the individual school or districts. While in Japan, I learned that students with special needs and learning disabilities were placed in special education facilities. These students 
were not immersed in the traditional classroom; they were housed in a facility, and the teachers worked in the facility. Twice a week, I was placed in a special education facility to work with the students and assist with introducing these students to society. For the first time, students were learning how to catch the train, how to go shopping, and how to mingle in the community. I developed a clear understanding of how the Japanese educational system valued the experience in the field as a key component of teacher development, and I developed a different perception about teacher preparation and my needs as a learner.

\section{Conclusion}

Although field-based teacher preparation programs are not new to the field of educational research, it is has sparked a growing interest over the past 20 years. Current research on perceptions of field-based teacher preparation programs discuss methods implemented to promote participants' dispositions (Austin, 2004; Seguin \& Ambrosio, 2002; Wasicsiko, 2005; Wilkerson \& Lang, 2007), perspectives of and early field experience in a laboratory setting (Washburn-Moses, Kopp, \& Hettersimer, 2012), and examining the possibilities offered through a campus/field-based teacher education course (Sanford \& Hopper, 2002).

This was the first and only inner city university in this state that implemented a field-based teacher preparation program in the mid-nineties. Unique to this specific university is the field-based teacher preparation, the social constructs of the program, and attributes of the community. The participants' prior corporate and leadership experiences, field-placements, relationships, professors, mentors, and community service all had a 
critical role in preparing graduates for the teaching profession. Despite these issues that directly or indirectly impact preparing teachers for the profession, the participants' recommendations should be considered in an effort to understand how to better prepare candidates for the teaching profession. The findings from this research may serve as a foundation to improve present and future field-based programs, its goals and objectives, university participation, school selection, and student enrollment in the program.

The sociocultural constructs of the field-based teacher preparation program provided an environment that was critical in relation to issues of language, cultural identity, and efficacy in addition to overall learning for the participants. The field-based teacher preparation program developed a higher level of professional identity and language among the participants and assisted them in embracing the school norms, culture, and methods of working, thinking, and developing as professionals. The data also suggests that the participants in the field-based teacher preparation program learned how to implement current educational practices in the classroom. The field-based program fostered continued professional development and positioned participants to further their education and seek other educational roles.

Researchers explicitly seek to examine the effectiveness of teacher preparation programs and field-experiences to identify if longer field experiences are adequately preparing teacher candidates for the teaching profession. Based on the findings of this study of graduates from an INC/FBTP with approximately 1-2 years of field-experience, participants learned how to effectively apply current educational practices in the classroom. Additionally, all of the participants indicated that the program provided them 
a clear understanding of the realities of being a teacher, the requirements necessary to secure employment, and how to develop as a school leader.

This research brings to the forefront the importance of participants' perceptions, preparation, collaboration, and disposition as it relates to field-based teacher preparation programs in the educative process by providing the experiences of actual participants from such program as told by the participants. It allowed participant to speak freely about their experiences in the various field-based schools, articulate what the participants believed enhanced their learning and share what identify their personal strength and weaknesses. Most importantly, participants were able to reflect on the overall field-based experiences and make recommendations for future programs. The data in this research communicates information that the participants felt necessary to share as it relates to their learning and development as professionals.

After an in-depth analysis of the major findings, the following key conclusions developed.

1. Field-based teacher preparation programs provided authentic classroom experience for teacher candidates, which allowed the participants to develop their own teaching philosophy, teaching strategies, and classroom management style.

2. Participants gained a plethora of knowledge and experience that prepared them for both student teaching and the teaching profession.

3. Participants developed unique relationships that assisted in their understanding of the culture of the school and community and the students they were teaching. 
4. Each participating school required that all field-based participants have the same expectations and respect as regular staff. This included participating in IEP meetings and staffing, completing IEPs, attending all staff meetings and professional development workshops and training. It is important that the academic community understand the significance of the participants experiences and perceptions while participating in such program to guide future program and address students' learning needs while in programs to and to assure that future field-based programs continue to align with the national guidelines.

To address the nation's challenge of preparing teachers for the teaching profession, it is critical to continue to analyze the experiences and perceptions of graduates from such programs as a means to inform future programs. This field-based teacher preparation program presented an innovative approach to teacher preparation programs that fostered collaboration, long-term mentoring, immersion in the classroom on the first day of school for the teachers, and weekly reflection. It offered longer authentic field-experience, flexibility, and resources to the participants of the program and it fostered collaboration among educators, participants, and community members. This field-based program presented a wide-range of cultural experiences that guided the participants' curriculum. This field-based program by no means provided one-size-fitsall solution to the future of teacher preparation, but it offered a starting point for navigating the intricate aspects of such programs designed to prepare teachers for the teaching profession. 


\section{Implication for Further Research}

This research was conducted during a major period of transformation in the $21^{\text {st }}$ century where a call for educational reform in teacher preparation standards and outcomes is always a topic of discussion in our nation's educational and political systems. Reform efforts will continue to change as long as academic and political agendas serve as a template for financial resolution. Future research might include an analysis of the placements of participants as it relates to authentic field-experiences, a comparative analysis of participant experiences in various field-based teacher preparation programs or professional development programs.

What remains to be explored is how participants' experiences differ from one another and if a longer duration of field-experience maximizes a participant's development as an educator or ultimately better prepares teachers for the teaching profession (Darling-Hammond, 2008). Differences in the participants' experiences included the location of the field-based school placement and grade levels; the relationships that developed among the professors; field-based participants and mentor teachers; how the participants gained information about the program; how information was communicated to the participants; and how the mentor teacher, university professors and field-based school principals, assessed participants. Each of these experiences was uniquely different for the participants. However, each of the participants articulated that they gained a higher level of appreciation for the teaching profession and that they were well prepared to serve in the role of an educator. Identifying these differences and fosters my interest in investigating deeper into specific differences through interview and 
observation in their own classrooms and videotaping participants during the data collection process.

Another possibility for future research includes an analysis of the experiences and perceptions of field-based professors, principals, and mentor teachers. Investigating the role of community service and professors in preparing teachers for the profession, or the availability of financial resources as it relates to program retention and the structure of the program as it relates to the social implications on the participants. There is a need to further this research by analyzing the perceptions of the participants to see if such programs are producing "Highly Qualified" teachers and to identify the social critical implications of such programs. It is critical to understand that participants' experiences and perceptions of the training they receive will assist in the implementation of future programs.

Additionally, the data is significant for current research on teacher preparation because it contains some of the key factors that researchers are analyzing to determine or validate teacher effectiveness as a result of participating in such programs, teacher retention rates, and areas of certification or endorsements for participants in field-based teacher preparation programs (Darling-Hammond, Wei, \& Johnson, 2009; DarlingHammond, 2010; Wilson, Floden, \& Ferrini-Mundy, 2001).

This research purports to close the gap in field-based teacher preparation research by providing current research on participants' experiences and perceptions as it relates to dispositions, preparedness, and relationships. Neither previous nor current research focuses on the participants' experiences or dispositions in teacher preparation. This 
research was designed to do that. It fills the gap in educational research on field-based teacher preparation programs regarding participants' experiences and perceptions regarding preparation for the teaching profession. It also provides recommendations from the field-based participants for future programs. It is informative, but not intended to be compared to professional development schools or other field-based teacher preparation programs. 


\section{REFERENCES}

Abel, C. (2002). Academic Success and the International Student: Research and Recommendations. New Directions for Higher Education, 2002, 117, 13-20.

ACEI-NCATE/CAEP Program Review. (2014) Retrieved on March 4, 2014, from http://www.acei.org/programs-events/acei-ncate/caep-program-review

Acquah, B. Y. S., \& Partey, A. T. (2014). The importance of field experience in teacher preparation: perspectives of trainee economics teachers in the University of Cape Coast. International Journal of Research In Social Sciences, 4,

Adams, A., Bondy, E., \& Kuhel, K. (Spring 2005). Preservice teacher learning in an unfamiliar setting. In S. Catapano \& S. Huisman, (2010). Perspectives on urban education. Teacher Education Quarterly, 32(2), 41-62

Agge, B. (2006). Critical social theories (2 ${ }^{\text {nd }}$ ed.). Herndon, VA: Paradigm Publishers.

Agee, J. (1998). Confronting issues of race and power in the culture of schools. In M. Dilworth (Ed.), Being responsive to cultural differences (pp. 21-38). Thousand Oaks, CA: Corwin Press.

Aggers, B. (2006). Critical social theories: An introduction ( $2^{\text {nd }}$ ed.). Boulder, CO: Paradigm Publishers.

Allsopp, D. H., DeMarie, D., Alvarez-McHatton, P., \& Doone, E. (2006). Bridging the gap between theory and practice: Connecting courses with field experiences. Teacher Education Quarterly [Online] Retrieved April 6, 2006, from http://www.findarticles.com/p/search

Alsup. J. (2006) Teacher identity discourses: Negotiating personal and professional spaces. Hillsdale, NJ: Lawrence Erlbaum Associates.

Álvarez, J. A., \& Bonilla, X. (2009). Addressing culture in the EFL classroom: A dialogic proposal. PROFILE Issues in Teachers' Professional Development, 11(2), 151-170.

American Association of Colleges for Teacher Education (AACTE). (2010, June 16-17). Reforming teacher preparation: The critical clinical component. Day on the Hill. Retrieved on September 8, 2011, from http://www.aacte.org 
American Association of Colleges of Teacher Education. (2010). The clinical preparation of teachers: A policy brief. Washington, DC: Author.

American Association of Colleges for Teacher Education. (2011, June 22). Transformations in educator preparation: Effectiveness and accountability. Retrieved September 8, 2011, from http://www.aacte.org

Anderson, L. M., \& Stillman, J. A. (2013). Students teaching contribution to preservice teacher development: A review of research focused on the preparation of teachers for urban and high-needs contexts. Review of Educational Research, 1(83), 3-69.

Andrews, D. J. C. (2009). The hardest thing to turn from the effects of service learning on preparing urban educators. Equity \& Excellence in Education, 42(3), 272-293.

Andrews, R. (2000). A prescription for improving teacher preparation: More action less abstraction. Education Week, 20, 37.

Anhorn, R. (2008). The profession that eats its young. Delta Kappa Gamma Bulletin, 74(3), 15-26.

Anke T., \& Korthagen, F. (2004). Deepening the exchange of student teaching experiences: Implications for the pedagogy of teacher education of recent insights into teacher behaviour. Teaching and Teacher Education, 20, 665-679.

Applegate, J. H. (1985). Early field experiences: Potpourri recurring. Journal of Teacher Education, 36(2), 60-64.

Applegate \& Lasley. (1985). Students' expectations for early field experience. Texas Technical Journal of Education, 12(1), 27-36.

Barksdale-Ladd, M. A. \& Rose, M. C. (1997). Qualitative assessment in developmental reading. Journal of College Reading and Learning, 28, 34-55.

Barth, R. (2001). Teacher Leader. Phi Delta Kappa, 443-449.

Basham, J. S. (2009). A study of perception of career American missionaries in the countries of Kenya and Tanzania relating to their overseas filed-based experiences.

Beatty, P. (2003, May). Paradigms of cognitive interviewing practice, and their implications for developing standards of best practice. Paper presented at QUEST (Questionnaire Evaluation Standards) 2003 Conference. Mannheim, Germany. Retrieved February 2, 2007, from http:www.gesis.org/Publi 
Beatty, P. (2004). The dynamics of cognitive interviewing. In S. Presser, J. M. Rothegeb, M. P. Couper, J. T. Lessler, E. Martin, J. Martin, \& F. Singer (Eds.). Methods for testing and evaluating survey results (pp. 45-66). New York, NY: Wiley.

Beck, C., Kosnik, C., \& Rowsell, J. (2007). Preparation for the first year of teaching: Beginning teachers' views about their needs. New Educator, 3(1), 51-73.

Beeth, M., \& Adadan, E. (2006). The influences of university-based coursework on field experience. Journal of Science Teacher Education, 17(2). 103-120.

Beisser, S. R (2000, April). Posing questions ... solving problems: Action research for preservice teachers. Paper presented at the annual meeting for the American Education Research Association, New Orleans, LA.

Beisser, S. R. (2008). Five-year study of field based pedagogical partnerships to prepare new elementary teachers. The Innovation Journal: The Public Sector Innovation Journal, 13(2), 3.

Bennett, S. V. (2010). Preservice teachers' developing understandings about culturally responsive teaching in a field-based writing methods course. ProQuest LLC, Ph.D. Dissertation, University of South Florida. 2010303 pp. (ED523363), Database: ERIC

Bergman, D. J. (2013). Comparing the effects of suburban and urban field placements on teacher candidates' experiences and perceptions of family engagement in middle and high schools. School Community Journal, 2012, (2), 23.

Best, S., \& Kellner, D. (1991). Post Theory: Critical interrogation. Critical perspective series. Guildford Press.

Blackledge, A., \& A. Creese. (2009). Meaning-making as dialogic process: Official and carnival lives in the language classroom. Journal of Language, Identity, and Education. 2009, 8, 4, 236-253.

Blackwell, P. J. (2002). Research, learning, and Paradoxes: The sixth annual Lanier lecture. Paper presented at the meeting of the Holmes Partnership Annual Meeting, San Antonio, TX.

Blaikie, N. (2007). Classical research paradigms. Approaches to Social Enquiry. Cambridge, MA: Polity Press.

Blaikie, N. (2009). Designing social research. Cambridge, MA: Polity Press.

Blaikie, N. (2012). Interpretivism: SAGE Research Methods. Retrieved on October 31, 2012, from http://srmo.sagepub.com/view 
Boas, F. (1995). Race, language, and culture. Chicago, IL: University of Chicago Press.

Boas, F. (1911). The mind of primitive man. A Course of Lectures Delivered before the Lowell Institute, Boston, MA, and the National University of Mexico, 19101911. New York, NY: Macmillan.

Boas, F. (1912). Changes in bodily form of descendants of immigrants. New York, NY: Columbia University Press.

Bogdan, R., \& Biklen (1982). Qualitative research for education: An introduction to theory and methods. Boston: Allyn and Bacon.

Bogdan, R., \& Taylor, S. J. (1982). The social construction of humanness: Relationships with severely disabled people. In P. M. Ferguson, D. L. Ferguson, \& S. J. Taylor (Eds.), Interpreting disability (pp. 275-294). New York: Teachers College Press.

Bransford (Eds.). Preparing teacher for a changing world (pp. 390-441). San Francisco, CA: Jossey-Bass.

Bratina, J. (2014). Peabody reports $20^{\text {th }}$ century education reform milestones. Vanderbilt community. The Vanderbilt View. Retrieved August 2014, from http://www.vanderbilt.edu/vanderbiltview.

Bruner, J. (1996). The culture of education. Cambridge, MA: Harvard University Press.

Burn, K., Hagger, H., Mutton, T., \& Everton, T. (2000). Beyond concerns with self: Sophisticated thinking of beginning student teachers. Journal of Education for Teaching, 26, 259-278.

Burns, B. A., Grande, M. L., \& Marable, M. A. (2008). Factors influencing teacher candidate's participation in a paid tutoring experience for urban students. Issues In Teacher Education, 17(2).

California State University Summit. (2011). Transformative change in the preparation of teachers. California State University. Retrieved on September 9, 2014, from http://www.calstate.edu/teacherEd/Summit/docs/CSU_Summit_Proceedings_032 211-acc.pdf

California State University. (BTSA) (2014). Online Master of Arts Education: Fieldbased. Retrieved on September 21, 2014, from http://www.csub.edu/maeducation/btsa_emph.html.

Capraro, M. M. (2005). Introduction to confidence intervals for both statistical estimates and effect sizes. Research in the Schools, 12(2), 13-23. 
Capraro, M. M. (2006a). Teaching portfolios: Technology skills + portfolio development. Do they powerful preservice teachers? Teacher Education and Practice, 19, 380-390.

Capraro, M. M. (2006b). Confidence intervals. In N. J. Salkind (Ed). The encyclopedia of measurement statistics. Thousand Oaks, CA: Sage

Capraro, R. M., Carparo, R.M., Parker, D., Kulum, G., \& Raulerson, T. (2005). The mathematics content knowledge role in developing preservice teachers' pedagogical content knowledge. Journal for Research in Childhood Education. $20,108-124$.

Capraro, M., Carparo, R. M., \& Helfeldt, J. (2010). Do differing types of field experiences make a difference in teacher candidates' perceived level of competence? Teacher Education Quarterly.

Capraro, R. M., Capraro, M. M., Parker, D., Kulm, G., \& Raulerson, T. (2005). The mathematics content knowledge role in developing preservice teachers' pedagogical content knowledge. Journal for Research in Childhood Education, $20,108-124$.

Carnegie Forum on Education and the Economy. (1986). A nation prepared: Teachers for the $21^{\text {st }}$ century. The Report of the Task Force on Teaching as a Profession.

Carnegie Forum on Education and the Economy. (2003). Task force on teaching as a profession. Toward a national workforce education and training policy.

Catapano, S., Huisman, S., \& Song, K. H. (2008). Are you there yet? Perspectives from partners in a community of practice. Learning Communities: International Journal of Learning in Social Contexts.

Charmaz, K. (2006). Constructing grounded theory: A practical guide through qualitative analysis. Thousand Oaks, CA: Sage.

Clarken, R. H. (1993). Clinical and field-based experiences: To prepare teachers for wholistic practice. Paper presented at the Annual Meeting of the American Educational Research Association, Atlanta, GA, April 12-16).

Cochran-Smith, M. (2005). Studying teacher education: What we know and need to know. Journal of Teacher Education, 56(4), 301-306.

Cochran-Smith, M., \& Zeichner, K. (Eds.). (2005). Studying teacher education: The report of the AERA Panel on research and teacher education. Mahwah, NJ: Lawrence Erlbaum Associates.

Common Standard for Educator Preparation. (2011). Retrieved August 2014 from 
http://www.teac.org/wp-content/uploads/2011/03/CAEP-standards.pdf

Conteh, J., Martin, P., \& Helavaara, R. L. (2008). Multilingual learning: Stories from schools and communities in Britain. Trentham: Stoke-on-Trent.

Conaway, B. J., Browning, L. J., \& Purdum-Cassidy, B. (2007). Teacher candidates' changing perceptions of urban schools: Results of a 4-year study. Action in Teacher Education, 29(1), 20-31

Cookson, P. W. (2005). Your first-year: Why teach? Teaching Pre K-8, 36(3), 14-21.

Cooper, R., Fleischer, A., \& Cotton, F.A. (2012). Building connections: An interpretative phenomenological analysis of qualitative research students' learning experiences. The Qualitative Report, 17, 1-16. Retrieved from http://www.nova.edu/ssss/QR/QR17/cooper.pdf

Council for Accreditation of Education Preparation. (2014). Retrieved March 2014 from caepnet.org

Crawford, B. A. (2007). Learning to teach science as inquiry in the rough and tumble of practice. Journal of Research in Science Teaching, 44, 613-642.

Creswell, J. W., \& Miller, D. (2000). Determining validity in qualitative inquiry. Theory into Practice, 39(3), 124-130

Creswell, J. W. (2014). Research design; Qualitative, quantitative and mixed methods approaches ( $3^{\text {rd }}$ ed.). Thousand Oaks, CA: Sage Publications.

Cristol, D. S., \& Gimbert, B. G. (Fall 2002). A case study of an urban school-university partnership: Designing and implementing curriculum for contextual teaching and learning. The Professional Educator, 15(1), 43-54. Retrieved from $\mathrm{http} / / /$ caepnet.org/resources/fall-2014-caep-conference-presentations/

Dacus, M. R., (1995). Giving voice to first-year teaches about their preparation for multicultural education: A grounded theory. ETD collection for the University of Nebraska-Lincoln. Paper AA19614982. http//digitalcommons.unl.edu/dissertations/AA19614982

Darling-Hammond, L. (2006). Powerful teacher education. San Francisco, CA: JosseyBass.

Darling-Hammond, L. (2007). A marshall plan for teaching. Education Week, 26(18), 4850 .

Darling-Hammond, L. (2010). Constructing $21^{\text {st }}$-century teacher education. In V. Hill- 
Jackson \& C. W. Lewis (Eds.), Transforming teacher education: What went wrong with teacher training and how we can fix it (pp. 223-247). Sterling, VA: Stylus.

Darling-Hammond, L. (2010). Recruiting and retaining teachers: Turning around the race to the bottom in high-need schools. Journal of Curriculum and Instruction (joCI), 4(1), 16-32. Retrieved from http://joci.ecu.edu.

Darling-Hammond, L., Chung, R., \& Frelow, F. (2002). Variation in teacher: How well do different pathways prepare teachers to teach? Journal of Teacher Education, 53(4) 286-302.

Darling-Hammond, L., Austin, K., Lit, I., \& Nasir, N. (2003). The classroom mosaic: Culture and learning. Stanford University School of Education, 105-124.

Darling-Hammond, L., \& Berry, B., (2006). Highly qualified teachers for all. Educational Leadership, 64(3), 14-20.

Darling-Hammond, L., Wei, R. C., \& Johnson, C. M. (2009). Teacher preparation and teacher learning: A changing policy lands CAEP. In G. Sykes (Ed.). The handbook of education policy research. Washington, DC: American Education Research Association.

Darling-Hammond, L. (2007). In K. Zeichner, K. (2010). Rethinking the connections between campus courses and field experiences in college- and university-based teacher education. Journal of Teacher Education, 61, 89.

Dean J. (1996). Beginning teaching in the secondary school. Buckingham: Open University Press.

Denzin, N. K., \& Lincoln, Y. S. (2012). Handbook of qualitative research $\left(2^{\text {nd }}\right.$ ed.). Thousands Oaks, CA: Jossey-Bass.

Dewey, J. (1938). Experience and education. New York, NY: Macmillan

Dinsmore, J., \& Wenger, K. (2006). Relationships in preservice teacher preparation: From cohorts to communities. Teacher Education Quarterly.

Dokko, G., Wilk, S. L., \& Rothbard, N. P. (2009). Unpacking prior experience: How career history affect job performance. Organization Science, 20(1), 51-68. Retrieved from hppt://journals.informa.org/

Douglas, J. (1976). Investigative social research: Individual and team field research. Beverly Hills, CA: Sage. 
Douglas, J. (1985). Creative interviewing. Beverly Hills, CA: Sage

Duncan, A. (2009). Teacher preparation: Reforming the uncertain profession.

Remarks at teachers college, Columbia University. Retrieved on March 4, 2010, from http://www.ed.gov/print/news/speeches

/2009/10/10222009.html

Duquette, C. (1997). Conflicting perceptions of participants in field-based teacher education programs. McGill Journal of Education, 32(3), 263-272.

Education Commission of the States. (2008). Eight questions on teacher preparation: What does the research say? Retrieved on October 15, 2008, from esc.org/html/education Issue

Edwards, S., \& Kuhlman, W. (Summer 2007). Culturally responsive teaching: Do we walk our talk? Multicultural Education, 14(4), 45-49.

Erickson, F. (1996). Qualitative methods in research on teaching. In M. C. Whittrock (Ed), Handbook of research on teaching (pp. 150-152). Old Tappan, NJ: Macmillan.

Finnan, C. (2000). Implementing school reform models: Why is it so hard for some schools and easy for others? Paper presented at the meeting of the American Educational Research Association, New Orleans. (ERIC Document Reproduction Service No. ED446356)

Fleet, M. (2006). The role of culture in second or foreign language teaching: Moving beyond the classroom experience. Retrieved from ERIC database. (ED491716)

Foote, C., \& Cook-Cottone, C. (2004). Field experiences in high-need, urban settings: Analysis of current practice and insights for change. Urban Review, 36(3), 189211.

Fletcher, S. S., \& Luft, J. A. (2011). Early career secondary science teachers: A longitudinal study of beliefs in relation to field experiences. Science Teacher Education, 1126-1146

Flores, M. A. (2007). Person and context in becoming a new teacher. Journal of Education for Teaching, 27, 135-148.

Franco, Z. (2006). Learning to plan for teaching: A multiple-case study of field-based experiences of three preservice teachers. The University of Alabama. Retrieved July 27, 2012, from http://gradworks.umi.com/32/23/3223337html

Freire, P. (2005). Education for critical consciousness. Continuum International Publishing Group. 
Freire, P. (1993). A critical encounter. New York: Routledge Publishing.

Friends School. (2014). Teacher preparation: Field-based apprentice model. Retrieved on October 4, 2014, from http://www.friendsschoolboulder.org/page/Programs/Teacher-PreparationProgram

Gadamer, H. G. (1975). Truth and method. New York: Seabury Press.

Gadamer, H. G. (1990/1960). Truth and method (2 ${ }^{\text {nd }}$ rev. ed). New York: Crossroad. Partnership: An exploratory qualitative case study.

Geertz, C. (1973). The interpretation of culture. New York, NY: Basic Books

George, P., \& Aronson, R. (2002). How do educators' cultural belief systems affect undeserved students' pursuit of postsecondary education? Briefing papers. Pacific resources for education and learning (PREL), Hawaii. Available at http://www.prel.org/products/pn_/cultural-belief.htm.

Gerring, J. (2003.) Symposium: Interpretations of interpretivism. Journal of Qualitative Methods, 2-6. Boston University.

Gersten, R., \& Baker, S. (2000). What we know about effective instructional practices for English-language learners. Exceptional Children, 66(4). 454-472.

Gibbs, G. R. (2007). Analyzing qualitative data. Thousand Oaks, CA: Sage.

Gimmett, P., \& Ratzlaff, H. (1986). Expectations for the cooperating teacher role. Journal of Teacher Education, 37, 41050.

Giorgi, A., \& Giorgi, B. (2008). Phenomenology. In. J. A. Smith (Ed.), Qualitative psychology: A practical guide to research methods (2 ${ }^{\text {nd }}$ ed., pp. 26-52). London: Sage.

Giorgi, A. (2009). The phenomenological method in psychology: A Modified Husserlian Approach. Pittsburgh PA: Duquesne University Press.

Giroux, H. A. (2011). On critical pedagogy. New York, NY: Bloomsbury Publishing.

Glaser, B. G. (1987). The sixties: Years of hope, days of rage. New York, NY: Bantam Books. 
Glaser, B. G., \& Strauss, A. S. (1967). The discovery of grounded theory: Strategies for qualitative research. New York, NY: Adline De Gruyter.

Glazer, E. M., \& Hannafin, M. J., (2006). The collaborative apprenticeship model: Situated professional development within school settings. Teaching and Teacher Education, 22(2), 179-193.

Gomez, M. L. (1996). Telling stories of our teaching, reflecting on our practices. Action in Teacher Education, 18, 1-12.

Goodlad, J. (1990). Teachers for our nation's schools. San Francisco, CA: Jossey-Bass.

Goodnough, K., Osmond, P., Dibbon, D., Glassman, M., \& Stevens, K. (2009). Exploring a triad model of student teaching: Pre-service teacher and cooperating teacher perceptions. Teaching and Teacher Education, 25(2), 285-296.

Goodwin, A. L. (2010). Globalization and the preparation of quality teachers: Rethinking knowledge domains for teaching. Teaching Education, 21(1), 19-32.

Grande, M., Burns, B., Schmidt, R., \& Marable, A. (2008). Impact of a paid urban field experience on teacher candidates' willingness to work in urban schools. Teacher Educator, 44(3), 188-203.

Grbich, C. (2013). Qualitative data analysis: An introduction. (2 ${ }^{\text {nd }}$ ed.). Thousand Oaks, CA: Sage Publications.

Griffin, G. (1999). Changes in teacher education: Looking to the future. The Education of Teachers. Chicago, IL: The National Society for the Study of Education.

Grimmett, P. (1988). The nature of reflection in Schon's conception in perspective. In P. Grimmett \& G. Erickson (Eds.), Reflection in teacher education (pp. 5-15). New York: Pacific Educational Press and Teachers College Press.

Grossman, P. (2010, May). Learning to practice: The design of clinical experience. Washington, DC: American Association of Colleges for Teacher Education \& National Education Association.

Guba E. G., \& Lincoln Y. S. (Eds.). (1994). Competing paradigms in qualitative research. Handbook of Qualitative Research. Thousand Oaks, CA: Sage Publications.

Guyton, E., \& Byrd, D. (Eds.). (1999). Standards for field experiences in teacher education. (1999). Task force on field-experience standards. Reston VA: Association of Teacher Educators. 
Guyton, E., \& McIntyre, J. D. (1990). Student teaching and field experiences. In W. R. Houston (Ed.), Handbook of Research on Teacher Education $\left(1^{\text {st }}\right.$ ed., pp. 514534). New York, NY: Macmillan.

Guyton, E., \& Wesche, M. (1996). Relationships among school context and student teachers' attitudes and performance. In J. D. McIntyre \& D. M. Byrd, Preparing tomorrow's teachers: The field experience. Thousand Oaks, CA: Corwin.

Habermas, J. (1971). Knowledge and human interests. Boston, MA: Beacon.

Haberman, M. (1995). Selecting 'star' teachers for children and youth in urban poverty. Phi Delta Kappan, 76(10), 777-782.

Haberman, M. (2004). Can star teachers create learning communities? Educational Leadership, 61(8), 52-56.

Hanline, M. F. (2010). Preservice teachers' perceptions of field experiences in inclusive preschool settings: Implications for personnel preparation. School of Teacher Education, Florida State University, Tallahassee, FL

Harty, H. (1979). A field-based professional development complex: One approach to future preservice-inservice teacher preparation. Peabody Journal of Education, 55(4), 337-45.

Hayes. D. (2009). Non-native English-speaking Teachers, Context and English Language Teaching. System, 37, 1-11.

Hayes, M. T. (2002). Assessment of a field-based teacher education program: Implications for practice. Academic Journal of Education, 122(4), 700.

Heidegger, M. (1962/1927). Being and time. Oxford: Blackwell.

Heimann, R. (2013). A dialogue forum of teachers and students as a resource of sustainability in a teacher education programme. Journal of Organisational Transformation \& Social Change, 10(2), 148-162.

Heinemann, H., Obi, R., Pagano, A., \& Weiner, L. (1995). Effects of using early preservice field experiences in urban settings to prepare teachers to meet the challenges of teaching in multicultural urban schools. Jersey City, NJ: Jersey City College. (ERIC Document Reproduction Service No. ED344868).

Heracleous, L., \& Henry, J (2000). Discourse and the study of organization: Toward a structurational perspective. Human Relations Journal, 53, 125. Retrieved on April 4, 2013, from http://hum.sagepub.com/cgi/content/abstract/53/10/1251

Hinchey, P. H. (1998). Finding freedom in the classroom: A practical introduction to critical theory. New York: Peter Lang Publishing, Inc. 
Hinde, E. R. (2004). School culture and change: An examination of the effects of school culture on the process of change. Arizona State University West. Retrieved on June 12, 2013, from www.usca.edu/essays/bp;122004/hinde.pdf

Hirsh, E. D. (1967). Validity in interpretation. New Have, CT: Yale University Press.

Hirschman, A. O. (1970). The search for paradigms as a hindrance to understanding. World Politics, 22(3), 329-43.

Hodgkinson, H. (2002). Demographics and teacher education. An overview. Journal of Teacher Education, 53(2), 102-105.

Hollins, E. R., \& Torres Guzman, M. (2005). Research on preparing teachers for diverse populations. In M. Cochran-Smith \& K. M. Zeichner (Eds.), Studying teacher education: The report of the AERA Panel on Research and Teacher Education. Mahwah, NJ: Lawrence Erlbaum.

Holstein, J. A., \& Gubrium, J. F. (1995). The active interview. Thousand Oaks, CA: Sage.

Hopper, T., \& Bell, R. (2014). In the beginning...Field based teacher education course in physical education: Impact on pre-service teachers and teacher educators. School of Physical Education, University of Victoria. Retrieved on September 13, 2014, from http://web.uvic.ca/ thopper/WEB/articles/fieldPE.htm

Huling, L. (1998). Early field experiences in teacher education. ERIC Digest. ERIC Clearinghouse on teaching and Teacher Education Washington DC. Retrieved on October 13, 2014, from http://ericae.net/edo/ed429054.htm

Humphrey, D. C., \& Wechsler, M. E. (2007). Insights into alternative certification: Initial findings from a national study. Teachers College Record, 109(3), 483-530.

Hung, H. T. (2008). Teacher learning: Reflective practice as a site of engagement for professional identity construction.US-China Education Review, 5(5), 39-49.

ISBE 23 Illinois Administrative Code. (2014). Section 25. Appendix D. Criteria for Identification of Teachers as "Highly qualified" in Various Circumstances.

Isaac, S., \& Michael, W. B. (1981). Handbook in research and evaluation. San Diego, CA: EDITS Publishers.

Ingersoll R. M. (2004). Why do high-poverty schools have difficulty staffing their classrooms with qualified teachers? Seattle, WA: University of Washington: Center for the Study of Teaching and Policy. 
Jones, M. G., \& Carter, G. (2007). Science teacher attitudes and beliefs. In S. K. Abell \& N. G. Lederman (Eds.), Handbook of research on science education (pp. 10671104). Mahwah, NJ: Lawrence Erlbaum Associates.

Kaelin, B. (2013). Preservice teacher perspectives on field experience, the development of teacher identity, and professional practice. Doctor of Education (EdD). http://digital commons.georgefox.edu/edu/29

Kastens, K. A., \& Liben, L. S. (in preparation). Children's strategies and mistakes in positioning field-based observations onto a basemap. For submission to Cognition \& Instruction.

Kellner, D. (2000). New technologies / new literacies: Reconstructing education for the new millennium. Teacher Education, 11(3), 245-265.

Kent, A., \& Simpson, J. L. (2009). Preservice teacher institute: Developing model learning community for student teachers. College Student Journal, 43(2), 695704.

Kim, K. (2005). Discourses of liberation: An anatomy of critical theory. Boulder, CO: Paradigm Publications.

Klein, H. K., \& Myers, M. D. (1999). A set of principles for conducting and evaluating interpretive field studies in information systems. MIS Quarterly, 23(1), 67-94. http://dx.doi.org/10.2307/249410

Klausewitz, S. K. (2005). How prior life experiences influence teaching: Multiple case studies of mature-age elementary student teachers. Electronic Doctoral Dissertations for UMass Amherst. Paper AAI3179892. Retrieved on May 30, 2013 from http://scholarworks.umass.edu/dissertations/AAI3179892

Knight, S. L., Nolan, J., Lloyd, G., Arbaugh, F., Edmondson, J., \& Whitney, A. (2013). Quality teacher education research: How do we know it when we see it? Journal of Teacher Education, 64(2).

Koeppen, K., Huey, G., \& Conner, K. (2000). Are effective model in a restructured teacher education program. In D. M. Byrd \& D. J, McIntyre (Eds.), Research on professional development schools. Thousand Oaks, CA: Crown Press.

Koerner, M. E., \& Abdul-Tawwab, N. (2006). Using community as a resource for teacher education: A case study. Equity \& Excellence in Education. 39, 37-46.

Korthagen, F. A., Loughran, J. J., \& Russell, T. (2006). Developing fundamental principles for teacher education programs and practices. Teaching and Teacher Education 22, 1020-1041. 
Kraft N. (2001). Standards in teacher education: A critical analysis of NCATE, INTASC, and NBPTS (a conceptual paper/review of research. Paper presented at the annual meeting of the American Education Research Association, Seattle, WA. (ERIC Document Reproduction Service No. ED462378)

Kramsch, C. (1993). Context and culture in language teaching. Oxford: Oxford University Press.

Kvale, S. (1996). Interviews: An introduction to qualitative research interviewing. Thousand Oaks, CA: Sage.

Kvale, S. (2009). Interviews: Learning the craft of qualitative research interviewing ( $2^{\text {nd }}$ ed.). Thousand Oaks, CA: Sage Publication

Kvale, S., \& Brinkmann, S. (2007). Doing interviews: The SAGE qualitative research kit. Thousand Oaks, C: Sage Publications, Inc.

Ladson-Billings, G. (1994). What we can learn from multicultural education research. Educational Leadership, 51(8). 22-26.

Ladson-Billings, G. (2006). It's not the culture of poverty, it's the poverty of culture. The problem with teacher education. Anthropology \& Education Quarterly, 37(2), 104-9.

Ladson-Billings, G. (2000). Fighting for our lives: Preparing teachers to teach African American students. Journal of Teacher Education, 51(3), 206-214.

Ladson-Billings, G. (2005). Is the team all right? Diversity and teacher education. Journal of Teacher Education, 56(3), 229-234.

Larkin, M., Watts, S., \& Clinton, E. (2006). Giving voice and making sense in interpretative phenomenological analysis. Qualitative Research in Psychology, 3, 102-120,

Latham, N. I., \& Vogt, P. W. (2007). Do professional development schools reduce teacher attrition? : Evidence from a longitudinal study of 1,000 graduates. Journal of Teacher Education, 58(2), 153-167. Retrieved from http://jte.sagepub.com/cgi/content/abstract/58/2/153T

Leland, C. H., \& Harste, J. C. (2005). Doing what we want to become. Preparing new urban teacher. Urban Education, 40(1), 60-67. 
Lemberger, J., Hewson, P. W., \& Park H. (1999). Relationships between prospective secondary teachers' classroom practice and their conceptions of biology and teaching science. Science Education, 83, 347-371.

Leonardo, Z. (2004). The color of supremacy: Beyond the discourse of "white privilege." Educational Philosophy and Theory, 36(2), 137-152

Leonardo, Z. (2004). Critical social theory and transformative knowledge: The functions of criticism in quality education. Educational Researcher, 33, 11. Retrieved from http://edr.sagepub.com/content/33/6/11

Lenski, S. D., Grisham, D. L., \& World, L. (Eds.). (2006). Literacy teacher preparation. Ten truths teacher educators need to know. Newark DE: International Reading Association.

Lenski, S. D., Crawford, K., Crumpler, T. and Stallworth, C. (2005). Preparing preservice teachers in a diverse world. Action in Teacher Education. 27(3), 3-12. Retrieved Sept. 2012 from https://www.academia.edu/1297072/Preparing_preservice_teachers in_a_diverse_world

Lester, S. (1999). An introduction to phenomenological research. Taunton, UK: Stan Lester Developments. Retrieved on September 12, 2012, from www.sld.demon.co.uk/resmethy.pdf.

Levine, M. (1997). Can professional development schools help us achieve what matters most? Action in Teacher Education. 19, 63-73.

Levinson, B. A. U. (2007). Critical social theories and education: Questions and possibilities.

Lincoln Y. S. \& Guba E .G. (1985). Naturalistic inquiry. Thousand Oaks, CA: Sage.

Lincoln Y.S., Guba E.G. (2000). The only generalization is: there is no generalization. In: R. Gomm, M. Hammersley, \& P. Foster (Eds.), Case study method. London: Sage Publications

Luft, J. A., Fletcher, S., \& Roehrig, G. (2007). Capturing science teacher's epistemological beliefs: The development of the teachers' beliefs interview. Electronic Journal of Science Education. 11(2). http://ejse.southwestern.edu/ volumes/v11n2/articles/art03._luft.pdf.

Lutz, F. W. \& Lutz, S. B. (1988). Organizational structure and loos coupling in higher education. Journal of Research and Development in Education. 21(2), 36-44. 
Lytra, V. (2011). Negotiating language, culture, and pupil agency in complementary school classrooms. Linguistics and Education, 3(1), 1-14.

Lytra, V., \& Barac, T. (2009). Multilingual practices and identity negotiations among Turkish-speaking young people in a diasporic context. In A.-B. Stedström \& A. Jorgensen (Eds.), Young speaking a multilingual perspective (pp. 55-78). Amsterdam/Philadelphia: Benjamin.

Mauthner, M, Birch, M. Jessop, J., \& Miller, T. (Eds.). (2002). Ethics in qualitative research. Thousand Oaks, CA: Sage.

MacDonald, C., McKinnon, M. Joyce, M., \& Gurney, P. (1992). Institutional constraints and role expectations: Perspectives on field experiences in an on-site teacher education program. McGill Journal of Education, 27, 45-59

McIntyre, D. J. (1983). Field experiences in teacher education: From student to teacher. Washington, DC: Foundations for Excellence in Teacher Education and ERIC Clearinghouse on Teacher Education.

MacIntyre, A. (1971). Is a science of comparative politics possible?" In Against the SelfImages of the Age: Essays on Ideology and Philosophy. London: Duckworth.

McLaren, P. (1994). Critical pedagogy: Constructing an arch of social dreaming and a doorway to hope. In L. Erwin \& D. MacLennan (Eds.), Sociology of education in Canada: Critical perspectives on theory research and practice (pp. 137-160). Toronto: Copp Clark Longman.

McLaren, P. (1989). Life in Schools: An introduction to critical pedagogy in the foundations of education ( $5^{\text {th }}$ ed.). New York, NY: Pearson Education, Inc.

McWilliam, E. (1994). In broken images: Feminist tales for a different teacher education. New York and London: Teachers College, Columbia University.

Melnick, S. A., \& Meister, D. G. (2008). A comparison of beginning and experienced teachers' concerns. Educational Research Quarterly, 31(3), 39-56.

Mertens, D. M. (2005). Research and evaluation in education and psychology: Integrating diversity with quantitative, qualitative, and mixed methods ( $2^{\text {nd }} \mathrm{ed}$.). Thousand Oaks, CA: Sage Publication, Inc.

Meyer, J., \& Rowan, B. (1975). The structure of educational organizations. In J. V. Baldridge \& T. Deal, (Eds.), The dynamics of organizational change in education. Berkeley, CA: McCutchan.

Miles, M., Huberman, M., \& Saldana, J. (2014). Qualitative data analysis: A methods sourcebook $\left(3^{\text {rd }}\right.$ ed.). Thousand Oaks, CA: Sage Publications. 
Moore, R. (2003). Reexamining the field experiences of pre-service teachers. Journal of Teacher Education, 54(1), 31-42.

Moore, L., \& Sampson, M. B. (2008). Field-based teacher preparation: An organizational analysis of enabling conditions. Education, 129(1).

Moran, D (2000). Introduction to phenomenology. London: Routledge.

Murshidi, R., Konting, M. M., Elias, H., \& Fooi, F. S. (2006). Sense of efficacy among beginning teachers in Sarawak. Teaching Education, 17(3), 265-275.

Nahal, S. P. (2010). Voices from the field: Perspectives of first year teachers on the disconnect between teacher preparation programs and the realities of the classroom. Research in Higher Education Journal.

Nahal, S. P. (2010). Exploring disparities between teachers' expectations and the realities of the education profession.

National Academy of Education. (2009). Teacher quality: Education policy white paper. Washington, DC: Author

National Center on Education and the Economy. (2014). Retrieved on November 27, 2014, from http://www.ncee.org/

National Center for Education Statistics (NCES). (2000). Teacher perceptions of students and parent problems. Washington, DC: U.S. Department of Education, Office of Educational Research and Improvement.

National Center for Education Statistics (NCES). (2003). Mini-digest of education statistics 2003. Retrieved on October 15, 2014, from http://nces.ed.gov /pubs2005/200517.pdf

National Council for Accreditation of Teacher Education (NCATE). (1998). Designing standards that work for professional development schools. Commissioned Papers of the NCATE PDS Standards Project. Washington, DC: Author.

National Council for Accreditation of Teacher Education (NCATE). (2002). Professional standards for the accreditation of schools, colleges, and departments of education. Washington, DC: Author.

National Council for Accreditation of Teacher Education (NCATE). (2007). NCATE defines professional dispositions as used in teacher education NCATE and social justice: A call to action. Washington, DC: Author. 
National Council for Accreditation of Teacher Education (NCATE). (2008a). Why did NCATE develop standards for professional development schools? Retrieved May 28, 2008, from http://www.ncate.org

National Council for Accreditation of Teacher Education (NCATE). (2008b). Mission, number of accredited institutions, process, history, influence.

Retrieved October 3, 2008, from http://education.stateuniversity.com/

National Council for Accreditation of Teacher Education (NCATE) (2010).

Transforming teacher education through clinical practice: A national strategy to prepare effective teachers. Report of the Blue Ribbon Panel on Clinical Preparation and Partnerships for Improved Student Learning. Retrieved from http://www.ncate.org

National Research Council. (2010). Preparing teachers: Building evidence for sound policy. Committee on the Study of Teacher Preparation Programs in the United States, Center for Education. Division of Behavioral and Social Sciences and Education. Washington, DC: The National Academies Press.

NCLB. (2002). No Child Left Behind Act, 2001. Ed. Gov. Retrieved April 1, 2006, from http://www.ed.gov/policy/elsec/leg/esea02/index.html

New Commission on the Skills of the American Workforce. (2009). The opportunity equation'- Sees intersection of interest mobilizing for education reform.

Retrieved on August, 2009 from http://www.skillscommission.org/?page_id=234

Nieto, S. (2002). Language culture and teaching: Critical perspectives for a new century. London, UK: Lawrence Erlbaum Associates.

Norris, S. (2009). Mediated discourse and social interaction: A reflection. In I. Stockburger \& J. Jia Lou (Eds.), A special issue in honor of Ron Scollon (pp. 3233). eVox, 4(1).

Norris, S., \& Jones, R.H. (Eds.). (2005). Discourse in action: Introducing mediated discourse analysis. London: Routledge

North East Texas. (2013). Field-based teacher education program handbook. Preparing tomorrow's teachers for today. Retrieved on September 21, 2014, from http://www.tamuc.edu/academics/colleges/educationHumanServices/educatorCert ificationAcademicServices/documents/fieldbased.pdf

Obama, B. (2009). Remarks by the president to the Hispanic Chamber of Commerce. Retrieved September 13, 2012, from gttp://www.whitehouse.gov/the_press_office/remarks-of-the-president-to-theUnited-States-Hispanic-Chamber-of-Commerce 
Our future, our teachers: The Obama Administration's Plan for Teacher Education Reform and Improvement. (2011). United State Department of Education. Retrieved August 2014 from http://www.ed.gov

Pagano, A., Weiner, L., Obi, R., \& Swearingnen, J. (1995). How student teaching in an urban setting affects teacher candidates' career motivations. The Urban Review, 27(1), 51-76.

Paige, R. M., Jorstad, H., Siaya, L., Klein, F., \& Colby, J. (2003). Culture learning in language education: A review of the literature. In R. M. Paige, D. L. Lange, \& Y. A. Yershova (Eds.), Culture as the core: Integrating culture into the language curriculum (pp. 47-113). Minneapolis, MN: University of Minnesota.

Patton, M. (2002). Qualitative research \& evaluation methods ( $3^{\text {rd }}$ ed.). Thousand Oaks, CA: Sage

Perry, A. (2011). Teacher preparation programs: a critical vehicle to drive student achievement. re:vision. California: The Hunt Institute.

Perry, C. (2004). Finding the truths in teacher preparation field experiences. Retrieved April 1, 2006, from http://www.findarticles.com

Pence, H. M., \& McGillivray, I. K. (2008). The impact of an international field experience on preservice teachers, 24(1), 14-25.

Penn State University-State College Area School District Professional Development School Partnership. (2014). Retrieved on November 13, 2014, from http://www.ed.psu.edu/pds/

Proctor, T. J., Rentz, N. L., \& Jackson, M. W. (2001). Preparing teachers for urban schools: The role of field experiences. The Western Journal of Black

Studies, $\quad$ 25(4), 219-226.

Prasad, P. (2005). Crafting qualitative research: Working in the post positivist traditions. Armonk, NY: M.E. Sharpe.

Putton, R. T., \& Borko, H. (2000). What do new views of knowledge and thinking have to say about research on teacher learning? Educational Researcher, 29(1), 4-15.

Radnor, H. (2002). Researching your professional practice: Doing interpretive research. City, State: Open University Press.

Rabinow, P., \& Sullivan, W. M. (1979). The interpretive turn: Emergence of an approach. Interpretive Social Science. Berkeley, CA: University of California Press. 
Raplay, T. (2007). Doing conversation. Discourse and documents analysis (Book 7 of the Sage Qualitative Research Kit). London: Sage

Rebar, C.R., Gersch, C. J., Macnee, C. L., \& McCabe, S. (2011). Understanding nursing research $\left(3^{\text {rd }}\right.$ ed.). London: Lippincott Williams \& Wilkins.

Reid, K., Flowers, P., \& Larkin, M. (2005). Exploring lived experience: An introduction to interpretative phenomenological analysis. The Psychologist, 18(1), 20-23.

Reed, L., Flowers, P., \& Larkin, M. (2006). Exploring lived experience. The Psychologist, 8(1), 20-23.

Repicky, P. A., \& Harty H. (1978). Evaluation design elements for an early field-based experience in science education for preservice teachers. Science Education, 59(4), 531-537, Oct-Dec 75. Retrieved Sept. 19, 2014, from http://0-

eric.ed.gov.opac.msmc.edu/?q=source $\% 3 \mathrm{a} \% 22 \mathrm{Science}+$ Education $\% 22 \& \mathrm{ff} 1=$ souS cience+Education \&ff2=autHarty\%2c+Harold\&id=EJ133354

Ritter, J. K, Powell, D., \& Hawley, T.S. (2007, January). Takin' it to the streets: A collaborative self-study into social studies field instruction. Paper presented at the Twentieth Conference of the Qualitative Interest Group, Athens, Georgia.

Robinson, M. (1999). ITE in a changing South Africa: experiences, reflections, and challenges. Exam Papers \& Reports. Paper 22. http://dk.cput.ac.za/ed_papers/22

Robinson, S., \& Darling-Hammond, L. (1994). Change for collaboration and collaboration for change: Transforming teaching through school-university partnerships. In L. Darling-Hammond (Ed.). Professional development schools: Schools for developing a profession (pp. 203-219). New York: Teachers College Press.

Rock, T. C., \& Levin, B. B. (2002). Collaborative action research projects: Enhancing preservice teacher development in professional development schools. Teacher Education Quarterly. 29(1), 7-21.

Rose, S. (2014). How do teacher preparation programs promote desired dispositions in candidates? Sage Open 2012. Retrieved on September 14, 2014, from http://sgo.sagepub.com

Ross, J. (1995) Professional development schools: Prospects for institutionalization Teaching and Teacher Education, 11, 195-20I.

Ross, F. (April 2001). So what type of teachers are they? Graduates of a PDS teacher preparation program 3-6 years later. Paper presented at the annual meeting of 
American Educational Research Association, Seattle, WA.

Rowlands, B. H. (2005). Grounded in practice: using interpretive research to build theory. Electronic Journal of Business Research Methods, 3(1), 81-92. Retrieved from www.ejbrm.com

Rubenstein, G. (2008). Building a better teacher: Confronting the crisis in teacher training. Edutopia. Retrieved May 3, 2008, from http://www.edutopia.org

Rubin, I. S. (1995). Qualitative interviewing: The art of hearing. Thousand Oaks, CA: Sage.

Saldana, J. (2013). The coding manual for qualitative researchers $\left(2^{\text {nd }}\right.$ ed.). Thousand Oaks, CA: Sage.

Salvato, N. (2005). Colleges of education urgently need reform, psychologist says. The Heartland Institute [Online]. Retrieved April 28, 2006, from http://www.hearland.org

Salzillo, F., \& Van Fleet, A. (1977). Student teaching and teacher education model for changer. Journal of Teacher Education, 28(1), 27-31

Sampson, M. B., Foote, M., Fleenor, C., \& Moore, L. (2001). Exploring the evolution of public school teachers' perceptions of university/public school partnerships: The quest for collaborative preparation of literacy teachers. Celebrating the voice of literacy: The twenty-third yearbook of the College Reading Association.

Sampson, M. B., Linek, W. M., Raine, I. L., \& Szabo, S. (2013). The influence of prior knowledge, university coursework, and field experience on primary preservice teachers \& apos. Use of Reading Comprehension Strategies in a Year-Long, Field-Based Teacher Education Program.

Sandberg, J. (2005). How do we justify knowledge produced within interpretive approaches? University of Queensland. Organizational Research Methods, 8(1), 41-68.

Sanford, K., \& Hopper, T. (2001). Field-based teacher education: Situating an initial teacher preparation course within the school context (Meeting Paper). Seattle, WA: American Educational Research Association.

Sanford, K., \& Hopper, T. (2002). Collective case study of a field-based teacher education course: A three-year action research project. Paper presented at the American Educational Research Association, New Orleans, LA. 
Sarason, S. B., Davidson, K. D., \& Blatt, B. (1986). The preparation of teachers: An unstudied problem in education. Cambridge, MA: Brookline Books.

Schleiermacher, F. (1998). Hermeneutics and criticism and other writings (A. Bowie, Trans.). In J. Smith, P. Flowers, \& M. Larkin, M. (2010). Interpretative phenomenological analysis: Hermeneutics and criticism and other writings. Cambridge. CUP

Schoeman, S., \& Mabunda P. L (2012). Teaching practice and the personal and socioprofessional development of prospective teachers. South African Journal of Education, 32, 240-254

Scotland, J. (2012). Exploring the philosophical underpinnings of research: Relating ontology and epistemology to the methodology and methods of scientific, interpretive, and critical research paradigms. Canadian Center of Science and Education. English Language Teaching, 5(9).

Schram, T. (2005). Conceptualizing and proposing qualitative research ( $2^{\text {nd }} \mathrm{ed}$.). Upper Saddle River, NJ: Merrill/Prentice Hall.

Schram, T. H. (2003). Conceptualizing qualitative inquiry: Mindwork for fieldwork in education. Upper Saddle River, NJ: Merrill/Prentice Hall.

Schuman, D. (1982). Policy analysis, education, and everyday life. Lexington, MA: Heath, Trans.). Chicago, IL: Northwestern University Press.

Schutz, A. (1999). Life world and cultural difference. In: Embree (Ed.), pp. 159-172.

Schutz, A. (1998). Phenomenological analysis and its contemporary significance. Human Studies, 21, 121-139

Schutz, A. (1967). The phenomenology of the social world (G. Walsh \& F Lenhert, Trans.). Evanston, IL: Northwestern University Press.

Schutz, A. (1962). Collect papers (vol. 1). The Hague: Martinus Nijhoff.

Schutz, A. (1953). Common-sense and scientific interpretation of human action. Philosophy and Phenomenological Research, 14(1), 3-47.

Schutz, A., \& Luckmann, T. (1974). The structures of the life-world. London: Heinemann Educational Books.

Schwandt, T. (1994). Constructivist, interpretivist approaches to human inquiry. In N. K. Denzin \& Y. S. Lincoln (Eds.), Handbook of qualitative research. Thousand Oaks, CA: Sage Publications 
Schwandt, T. (2001). Hermeneutic circle. In T. Schwandt (Ed.), Dictionary of qualitative inquiry (pp. 112-118). Thousand Oaks, CA: Sage.

Schwandt, T. A. (2005). Three epistemological stances for qualitative inquiry. In N. K. Denzin, \& Y. S. Lincoln (Eds.), Handbook of qualitative research ( $3^{\text {rd }} \mathrm{ed}$.). London: Sage Publications.

Schwandt, T. A. (2007). The SAGE dictionary of qualitative inquiry ( $\left.3^{\text {rd }} \mathrm{ed}.\right)$. Los Angeles, CA: Sage Publications.

Schwartz-Shea, P., \& Yanow, D. (2012). Interpretive research design: Concepts and processes. New York, NY: Taylor 7 Francis.

Segal, E. H. (2011). Early urban field experiences for prospective teachers: A case study of multicultural field placements through a university-based preservice STEM teacher program. ProQuest LLC.

Seidman, I. E. (2006). Interviewing as qualitative research: A guide for researchers in education and the social sciences. New York, NY: Teachers College Press

Sexton, R. (2008). Efforts to improve teaching get a new focus: Reforms rethink education's 'human capital system.' Prichard Committee for Academic Excellence. Perspectives, 19, 2.

Shanahan, C. H. (2008). Essential fieldwork for the preparation of teachers of reading for urban settings. In L. C. Wilkinson, L. M. Morrow, \& V. Chou (Eds.), Improving literacy achievement in urban schools: Critical elements in teacher preparation. Newark, DE: International Reading Association.

Shen, J. (1994). A study in contrast: Visions of preservice teacher education in the context of a professional development school. Paper presented at the Annual Meeting of the American Association of Colleges for Teacher Education, Chicago, February 16-19.

Shohamy, E. (2007). Language tests as language policy tools. Assessment in Education, 14(1), 117-130. Retrieved March 20, 2012, from https://cmap.helsinki.fi/servlet/SBReadResourceServlet?rid...1TD

Short, M. N. \& Bullock, L. M. (2013). Perspectives on select field-based experiences for pre-service teachers of students with emotional and behavioural disorders. Emotional \& Behavioural Difficulties, 18(4), 396-406.

Simpson, E. S., Yocom, D. J., \& Blum, H. T. (2006). The Wyoming collaborative mentorship. Academy: A Field-based Program for Certifying Fully qualified 
Special Education Teachers in a Rural State. Rural Special Education Quarterly, 24(2), 11-17.

Sleeter, C. E. (2001). Preparing teachers for culturally diverse schools: Research and the overwhelming presence of whiteness. Journal of Black Studies, 52(2), 94-106.

Smith, J. A. (2004). Reflecting on the development of interpretative phenomenological analysis and its contribution to qualitative research in psychology. Qualitative Research in Psychology, 1(1), 39-54.

Smith, J. A. (2011). Evaluating the contribution of interpretative phenomenological analysis. Health Psychology Review, 5(1), 9-27.

Smith, J. A. (2010). Interpretative phenomenological analysis: A reply to Amedeo Giorgi. Existential Analysis, 21 (2), 186-191.

Smith, J. A. (2011). Evaluating the contribution of interpretative phenomenological analysis. Health Psychology Review, 5, 9-27.

Smith, J. A. (2011). Evaluating the contribution of interpretative phenomenological analysis: A reply to the commentaries and further development of criteria. Health Psychology Review, 5, 55-61.

Smith, J. A. (2007). Hermeneutics, human sciences and health: Linking theory and practice. International Journal of Qualitative Studies on Health And Well-Being, 2, 3-11

Smith, J. A. (Ed.). Qualitative psychology: A practical guide to research methods ( $^{\text {nd }}$ ed., pp. 53-79). London: Sage.

Smith, J. A. (2009). Interpretative phenomenological analysis: Theory, method and research. Thousand Oaks, CA: Sage Publications.

Smith, J. A., Flowers, P., \& Larkin, M. (2009). Interpretive phenomenological analysis: Theory, method and research. London: Sage.

Smith, J. A., Flowers, P., \& Larkin, M. (2010). Interpretative phenomenological analysis: Theory Method and Research. London: Sage

Smith, J., Jarman, M., \& Osborne, M. (1999). Doing interpretative phenomenological analysis. In M. Murray \& K. Chamberlain (Eds.), Qualitative Health Psychology. London: Sage.

Smith J. A., \& Osborn, M. (2008). Interpretative phenomenological analysis. In 
J. A. Smith (Ed.), Qualitative psychology. A practical guide to research methods (pp. 51-80). London: Sage.

Smith, M. S. (2005). Helping preservice teaches develop habits of inquiry: Can it be done? Reading Research and Instruction, 45, 39-68.

Smith, P. L., Harris, C. M., Sammons, L., Waters, J., Jordan, D., Martin, D., Smith, N., \& Cobb, P. (2000). Using multimedia portfolios to assess preservice teachers and $P$ 12 student learning. (ERIC Document Reproduction Service No. ED445052)

Smith, A., Sanders, M., Norsworthy, B., Barthow, S., Miles, L., Ozanne, P., \& Weyderman, C., (2012). Maximising learning dialogue between workplace mentors and students undertaking professional field-based experiences. Wellington NZ. Retrieved from http://akoaotearoa.ac.nz/learning:dialogue-infield-based-experiences

Spradley, J. P. (1979). The ethnographic interview. New York, NY: Holt Rhinehart \& Watason.

Stivers, T., Enfield, N. J., \& Levinson, S. C. (2007). Person reference in interaction. Introduction. In N. J. Enfield \& T. Stivers (Eds.), Person reference in interaction: Linguistic, cultural, and social perspectives (pp. 1-20). Cambridge MA: University Press.

Stover, D. (2005). Climate and Culture: Why your board should pay attention to the attitudes of students and staff. American School Board Journal.

Study of Teaching and Policy. (2001). Retrieved September 30, 2010, from http://depts.washington.edu/ctpmail/PDFs/TeacherPrep-WFFM-02-2001.pdf

Swartz, E. (2003). Teaching white preservice teachers: Pedagogy for change. Urban Education, 36(3), 255-278.

Szente, J. (2008/2009). Academic enrichment programs for culturally and linguistically diverse children. Childhood Education, 85(2), 113-117.

Taylor, C. (1994). Interpretation and the sciences of man. In M. Martin \& L. McIntyre (Eds.), Readings in the Philosophy of Social Science. Cambridge, MA: MIT Press.

Taylor, Charles. (1985). Interpretation and the sciences of man. Philosophy and the Human Sciences: Philosophical Papers (vol. 2). Cambridge: Cambridge University Press.

Tableman, B. (2004). School culture and school climate: Best Practice Briefs. Ann Arbor, MI: Michigan State University. 
Tellez, K. (2008). What student teachers learn about multicultural education from their cooperating teachers. Teaching and Teacher Education, 24(1), 43-58.

The Journal of Curriculum and Instruction (JoCI). (2010). Recruiting and retaining teachers. Turning around the race to the bottom in high-need schools, 4(1), 1632. Retrieved September 12, 2014 from http://www.joci.ecu.edu

The National Council on Teacher Quality. (2011). Student teaching in the United States: Executive summary. Retrieved September 15, 2012, fromhttp://www.nctq.org/edschoolreports/studentteaching/executiveSummary.jsp

Thompson, B. (2005). "Statistical," "practical," and "clinical”: How many kinds of significance do counselors need to consider? Journal of Counseling and Development, 80, 64-71.

Thompson, S., \& Smith D. L. (2005). Creating highly qualified teachers of urban schools. Professional Educator, 27(1-2), 73-88.

http://files.eric.ed.gov/fulltext/EJ728483.pdf

University of Texas El Paso (2009). Undergraduate Catalog: College of Education: Field-Based Teacher Preparation. Retrieved September 9, 2014, from http://www.utep.edu/catalogs/1998ug/edu/field.htm

U. S. Department of Education. (2011). Our future, our teachers: The Obama administration's plan for teacher education reform and improvement. Washington, DC. Retrieved from http://www.2edgov/inits/ed/index/html.

U. S. Department of Education. (2009). Education Secretary Duncan announces $\$ 43$ million in grants to improve teaching in high-needs school. Retrieved March 4, 2010, from hhtp://www.ed.gov/news/press-releases

U.S. Department of Education. (2009). Education secretary launches national discussion on education reform. [Press release]. Retrieved March 4, 2010, from http://www2.ed.gov/news/pressreales/2009/05/05052009.html

Utley, C. A., Obiakor, F. E., \& Bakken, F. E. (2011). Culturally responsive practices for culturally and linguistically diverse students with learning disabilities. Learning Disabilities: A Contemporary Journal, 9, 5-18.

Utley, C. A. Delquadri, J. C., Obiakor, F. E., \& Mims, V. A. (2000). General and special educators' perceptions of teaching strategies for multicultural students. Teacher Education and Special Education, 23, 34-60. 
Varghese, M., Morgan, B., Johnson, B., \& Johnson, K. (2005). Theorizing language teacher identity: Three perspectives and beyond. Journal of Language, Identity, and Education, 4(1), 21-44.

Vygotsky, L. S. (1962). Thought and language (E. Hanfmann \& G. Vaker, trans.). Cambridge, MA: MIT Press.

Von Wright, G. H. (1971). Explanation and understanding. Ithaca, NY: Cornell University Press.

Waller, W. (1932). The sociology of teaching. New York, NY: Wiley.

Washburn-Moses, L., Kopp, T., \& Hettersimer, J. E. (2012). Prospective teachers' perceptions of the value of an early field experience in a laboratory setting. Issues in Teacher Education, 21(2).

Weber, M. (1947). The theory of a social science and its relation to philosophy. London: Routledge \& Kegan Paul.

Weber, M. (1949). The methodology of the social sciences. New York, NY: Free Press.

Weber, M. (1964). The theory of social and economic organization. New York, NY: Free Press.

Weber, M. (1978). The nature of social action. In W. Runcima (Ed.), Weber: Selection in transition. Cambridge, MA: Cambridge University Press.

Weber, M. (1978). Economy and society: An outline of interpretive sociology. G. Roth \& C. Wittich (Eds.). Berkeley: University of California Press.

Weber, S. J. (1996). The nature of interviewing. Phenomenology + Pedagogy, 4(2), 6572.

Weiner, L. (2002). Evidence and inquiry in teacher education: What's needed for urban schools. Journal of Teacher Education.

Wenzlaff, T., \& Wieseman, K. (2004). Teachers need teachers to grow. Teacher Education Quarterly, 31(2), 113-124.

Wertsch, J. (1991). Voices of the mind: A sociocultural approach to mediated action. Harvard University Press.

Westbrooks, S., \& Alea J. (2007). Mentor-based teacher preparation and student teacher performance. Illinois School Journal, 86(2), 42-55. 
Weiss, R. S. (1994). Learning from strangers: The art and method of qualitative interview studies. New York, NY: The Free Press.

Wiggins, R. A., Follo, E. J., \& Eberly, M. B. (2007). The impact of a field immersion program on pre-service teachers' attitudes toward teaching in culturally diverse classrooms. Teaching and Teacher Education, 23, 653-663.

Wikipedia, the free encyclopedia. (2014). Interpretative phenomenological analysis. Retrieved October 17, 2012, from http://en.wikipedia.org/wiki/Interpretative_phenomenological_analysis

Williams, E. (2012). Critical issues in literacy pedagogy; Notes from the trenches. University Readers, Inc.

Williams, M. (1998). The social world as knowable. In T. May \& M. Williams (Eds.). Knowing the Social World. Buckingham: Sociology, 34(2), 209-224. United Kingdom 2000 BSA Publications Limited Open University Press.

Wilmore, E. (1996). Brave new world: Field-based teacher preparation. Educational Leadership, 53(6), 59-63.

Wilson, S.M., Floden, R.E., \& Ferrini-Mundy, J. (2001). A Research Report prepared for the U.S. Department of Education and the Office for Educational Research and Improvement. The Center for the Study of Teaching and Policy in collaboration with Michigan State University

Wilson, S. M., Floden, R. E., \& Ferrini-Mundy, J. (2002). Teacher preparation research: An insider's view from the outside. Journal of Teacher Education, 53, 190-204.

Wilson, S. M., Floden, R. E., \& Ferrini-Mundy, J. (2011). Teacher preparation research: Current knowledge, gaps, and recommendations. Seattle, WA: The Center for the Study of Teaching and Policy in collaboration with Michigan State University.

Winch, P. (1970). The idea of a social science. In B. Wilson (Ed.), Rationality. Oxford: Blackwell.

Winch, P. (1990 /1958). The idea of a social science and its relation to philosophy ( $2^{\text {nd }}$ ed.). London: Rutledge \& Kegan Paul.

Wink, J. (2005). Critical pedagogy: Notes from the real world ( $3^{\text {rd }}$ ed.). Boston, MA: Person, Allyn \&Bacon.

Wink, J., \& Wink, D. (2004). In R. Phillipson (Ed.), Rights to language: Equity, power, and education. Mahwah, NJ: Lawrence Erlbaum Associates. 
Winitzky, N., Stoddart, T., \& O'Keefe, P. (1992). Great expectations: Emergent professional development schools. Journal of Teacher Education. 43(1). 3-18.

Wiseman, D. L., \& Nason, P.L. (1995). The nature of interactions in a field-based teacher education experience. Action in Teacher Education, 42(3), 1-12.

Wiseman, D. L. (2012). The intersection of policy, reform, and teacher education. Journal of Teacher Education, 63(2), 87-91.

Wolpert, E. M. (2010). Field based cohorts: The Teacher Education Programs At Georgia College \& State University: The Beginning. College of Education, Georgia College \& State University.

Wright, C. (2009). A study to explore the strategy of field-based teacher preparation: Professional development schools. Electronic Theses and Dissertations. http://dc.etsu.edu/etd/1841.

WSU College of Education Office of Field Services \& Certification. (2014). Field-based teacher preparation program

Yanow, D., \& Schwartz-Shea, P. (2006). Interpretation and method: Empirical research methods and the interpretive turn. New York: M.E. Sharpe, Inc.

Yanow, D. (2012). Interpretive research design: Concepts and process. New York, NY: Routledge, Taylor \& Francis Group Publishing.

Yarger, J. B., \& Howey, K. (1977). Preservice teacher education. Palo Alto, CA: Boosend Laboratory

Yusko, B., \& Feiman-Nemser, S. (2008). Embracing contraries: Combining assistance and assessment in new teacher induction. Teachers College Record, $110,123-953$

Zaid, M. A. (2011). 2011 International Conference on Languages, Literature and Linguistics IPEDR, 26. Singapore: IACSIT Press.

Zeichner, K. M. (1980). Myths and realities: Field-based experiences n preservice teacher education. Journal of Teacher Education, 21(6), 45-49, 51-55.

Zeichner, K. M. (1981). Reflective teaching and field-based experience in teacher education. Interexchange, Vol. 4.

Zeichner, K. M. (1982). Personalized and inquiry oriented teacher education. Journal of Education for Teaching, 8(20), 95-117. 
Zeichner, K. M. (1991). Reflective teaching and field-based experience in teacher education. Interexchange, 12(4), 1-22..

Zeichner, K. (1996). The role of community field experiences in preparing teachers for cultural diversity. In K. Zeichner, S. Melnick, M. L. \& Gomez (Eds.), Currents of reform in preservice teacher education (pp. 176-196). New York, NY: Teachers College Press.

Zeichner, K. M. (1999). The new scholarship in teacher education. Educational Researcher, 28(9), 4-15.

Zeichner, K. M. (2002). Does this fit? INTASC-based portfolios in a constructivist program. (ERIC Document Reproduction Service No. ED475826)

Zeichner, Z. (2010). Rethinking the connections between campus courses and field experiences in college-and university-based teacher education. Journal of Teacher Education, 61(1-2), 89-99.

Zeichner, K. M. S., \& Gomez, M. L. (1996). (Eds.). Current of reform in preservice teacher education. New York, NY: Teachers College Press.

Zeichner, K. M., \& Tabachnick, R. B. (1981). Are the effects of university teacher education "washed out" by school experiences? Journal of Teacher Education, 32(3), 7-11.

Zeus, L. (2004). Critical social theory and transformative knowledge: The functions of criticism in quality education. Education Researcher, 33(6), 1-8. 
APPENDIX A

\section{LETTER OF INTRODUCTION}


Dear Colleague:

My name is Cynthia L. Thompson, and I am a doctoral candidate under the tutelage of Dr. Eurvine Williams in the Department of Curriculum and Instruction at Illinois State University. I am conducting an interpretive phenomenological qualitative research to explore the perceptions of participants' experiences from an inner-city fieldbased teacher-preparation program.

This research project will be conducted during the spring of 2013. I will write an analysis about my dissertation and will share the findings with my dissertation committee and you, the participant. I may also present the results in an academic venue. I am requesting your participation in this research. Your participation would include three tape-recorded interviews of approximately 40 minutes to an hour each time. Specifically, I will ask you questions that relate to the following:

1. Perception of preparedness knowledge,

2. Skills and attitudes that are critical for success, and

3. Relationships and experiences with professors and mentor teachers.

Tape recording will be necessary so that I can construct a verbatim transcript to use in the analysis. All transcripts, notes, and information will be destroyed at the conclusion of the dissertation process. I will keep confidential whatever information you provide. No one other than me will have access to the information, and the information you provide will not be identified by your name. Additionally, you will not be identified by your real name (I will use a pseudonym) in the dissertation I construct. Only I will have access to the transcripts and notes. In the dissertation, I write, there may be some quotations from the interviews, but I will not use your real name and will endeavor to protect your identity so that you will experience no adverse effects for your honesty in the interviews and your willingness to participate.

Your participation is voluntary. There is no penalty for deciding not to participate. In addition, you are free to withdraw from participation at any time, for any reason, with no penalties of any sort. Please sign below and return this form if you understand what I am asking of you and if you are willing to participate.

Thank you,

Cynthia L. Thompson, ABD 
APPENDIX B

INTERVIEW QUESTIONS 


\section{Interview Questions \#1}

1. How do field-based participants' describe their experience as participant in an inner city neighborhood school?

2. How has participation in the Field-based teacher preparation program influenced your perception of the teaching profession?

3. How has your experience in the field-based program prepared you for the teaching profession?

4. How has your experience in the field-based program developed your knowledge, skills, and attitudes necessary for the teaching profession?

5. How did the relationships enhance teacher efficacy?

\section{Interview Questions \#2}

1. How did your day go? What time did you start school and how did the day end? Reconstruct a typical day when you go in the school.

2. What specific experiences did field-based participants' find beneficial in developing relationships and experiences with professors and mentor teachers?

3. Given what you have experienced as a field-based participant, how do you understand your role as an educator?

4. What value, if any has been derived from your participation in the field-based program?

5. What specific experiences did your mentor provide that better prepared you for the classroom?

\section{Interview Questions \#3}

1. Reflect back to the onset of the program, how you developed during the program and the overall qualifications necessary for teacher certification. How has the program prepared you for the teaching profession?

2. Reflecting back on all of the field-based experiences. Describe in detail experiences that were provided by the mentors, which prepared you for the classroom. What as that "Ah Haa!" moment that made you realize that yes, I am ready for the classroom.

3. Given what you have experienced as field-based participants, how do you understand their role as educators?

4. What did you find to be the strengths and weaknesses of the field-based teacherpreparation program or professional development school program?

5. What changes would you recommend to enhance the program?

6. Do you believe that your experience prepared you to teach as first-year teachers? 
APPENDIX C

PARTICIPANT INFORMED CONSENT LETTER 


\section{INFORMED CONSENT LETTER FOR VOLUNTARY PARTICIPATION}

1. I have read the description of the dissertation titled, Articulating the Experiences and Perceptions of Graduates from an Inner City Field-based Teacher Preparation Program (INC/FBTP) and understand what will happen in the research project.

2. I understand that the primary purpose of this research is to explore and interpret the experiences of graduates from an inner-city field-based teacher- preparation program to determine how they feel about the preparation program and, additionally, to investigate whether graduates viewed themselves as well prepared for the teaching profession because of participation in a field-based teacher-preparation program.

3. I understand that I may ask questions about the dissertation or my participation in it at any time, to the researcher, her dissertation chair, or the Illinois State University Research and Sponsored Programs Office at any time:

\begin{tabular}{|l|l|}
\hline Cynthia L. & Research Ethics and \\
Thompson & Compliance Office \\
tkf1992@yahoo.com & $309-438-8451$ \\
\hline
\end{tabular}

4. I understand that whatever information I provide will be kept confidential.

5. Cynthia L. Thompson have my permission to use the information gathered from the taped interviews and notes with the understanding that they will not reveal my name or other identifying information in any publications or reports.

6. I volunteer to participate in this research and understand that the interview will be tape-recorded to facilitate analysis of the data.

7. I will be interviewed by Cynthia $\mathrm{L}$. Thompson using a structured and semi structured format consisting of three interviews.

8. I have the right to review material prior to the final oral exam or other publication.

9. I understand that results from this survey will be included in Cynthia L. Thompsons' doctoral dissertation and may also be included in manuscripts submitted to professional journals for publication, and conferences.

10. I understand that because of the small number of participants', approximately six, there is a small risk that I may be identified as a participant of this study.

11. I am willing to participate and I am (18 years old or older by December 1, 2013). (For legal reasons, all participants' must be 18 years old or older.)

Signature of Participant

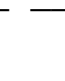

email 